\title{
Reduction Over Condensation of Carbonyl Compounds Through a Transient Hemiaminal Intermediate Using Hydrazine
}

Marcelo Vilches-Herrera, ${ }^{\dagger}$ Sebastián Gallardo-Fuentes,${ }^{\dagger}$ Mauricio Aravena-Opitz, ${ }^{\ddagger}$ Mauricio Yáñez-Sánchez, ${ }^{*}$ Haijun Jiao,' Jens Holz," Armin Börner,"¥ and Susan Lühr*,†,ł

${ }^{\dagger}$ Faculty of Sciences, University of Chile, Department of Chemistry, Las Palmeras 3425, 7800024, Ñuñoa, Santiago, Chile.

E-mail: susanluhr@uchile.cl

Faculty of Chemistry and Biology, University of Santiago of Chile, Department of Material Science, Av. Libertador Bernardo O’Higgins 3363, Santiago, Chile.

"Leibniz-Institut für Katalyse an der Universität Rostock e.V, Albert Einstein Str.29 a, 18059 Rostock, Germany. ${ }^{\ddagger}$ Institut für Chemie der Universität Rostock e.V., Albert Einstein Str. 3 a, 18059 Rostock, Germany.

\section{Table of Contents}

GC chromatogram after 24 hours of reaction using decane as internal standard

${ }^{1} \mathrm{H}-\mathrm{NMR}$ spectrum of $\mathbf{1 a}, \mathbf{2 a}, \mathbf{3 a}$ and $\mathbf{4 a}$ in THF $d_{8}$

Progress of the reaction followed by ${ }^{1} \mathrm{H}-\mathrm{NMR}$ spectrum

GC/MS response to equimolar concentrations of $\mathbf{2 a}, \mathbf{3 a}$ and $\mathbf{4 a}$

Computational methods and models

References

GC and GC-MS references

GC and GC-MS of chromatograms for all compounds 


\section{GC chromatogram after 24 hours of reaction using decane as internal standard}

Temperature program: $40{ }^{\circ} \mathrm{C}$ for $4 \mathrm{~min}$, then $15{ }^{\circ} \mathrm{C} / \mathrm{min}$ to $300{ }^{\circ} \mathrm{C}$ for $5 \mathrm{~min}$. Retention times: 9.565 min decane, $13.801 \mathrm{~min}$ alcohol $\mathbf{4 a}, 16.003 \mathrm{~min}$ hydrazone $\mathbf{2 a}, 22.59 \mathrm{~min} \mathbf{3 a}$.

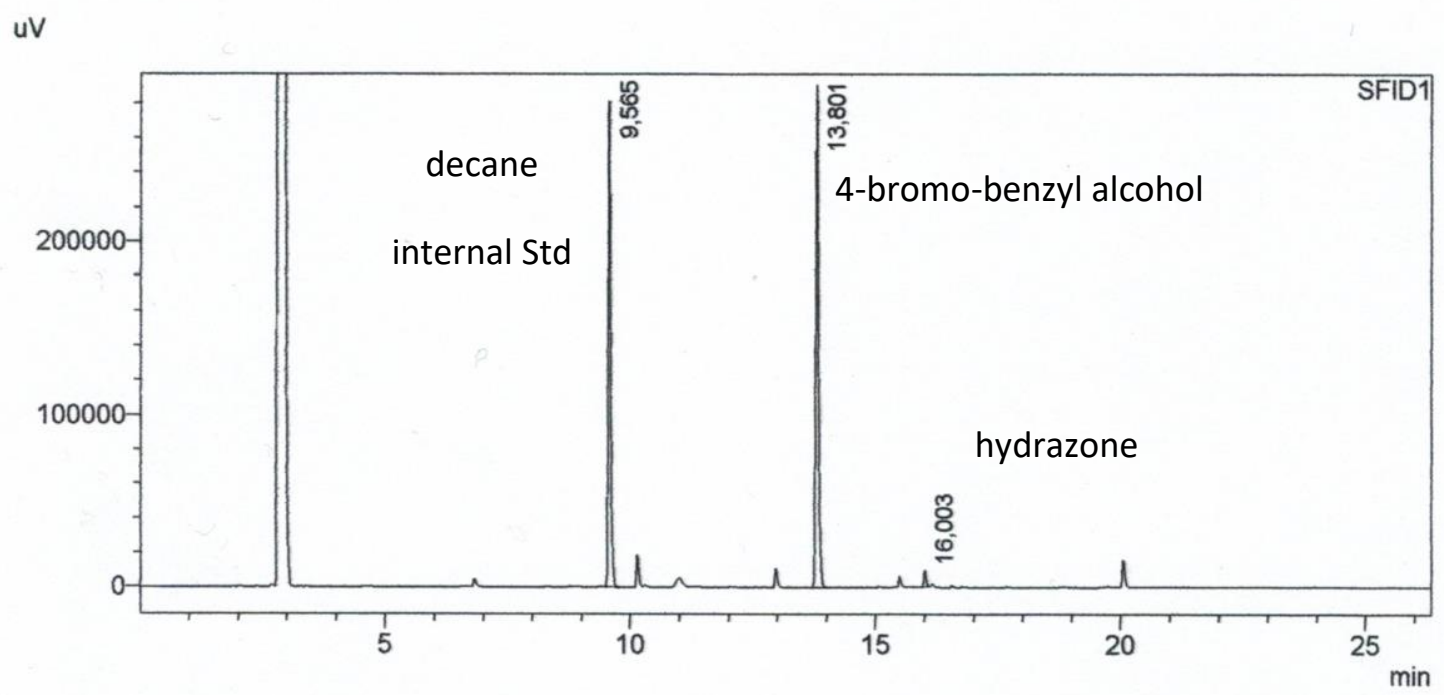




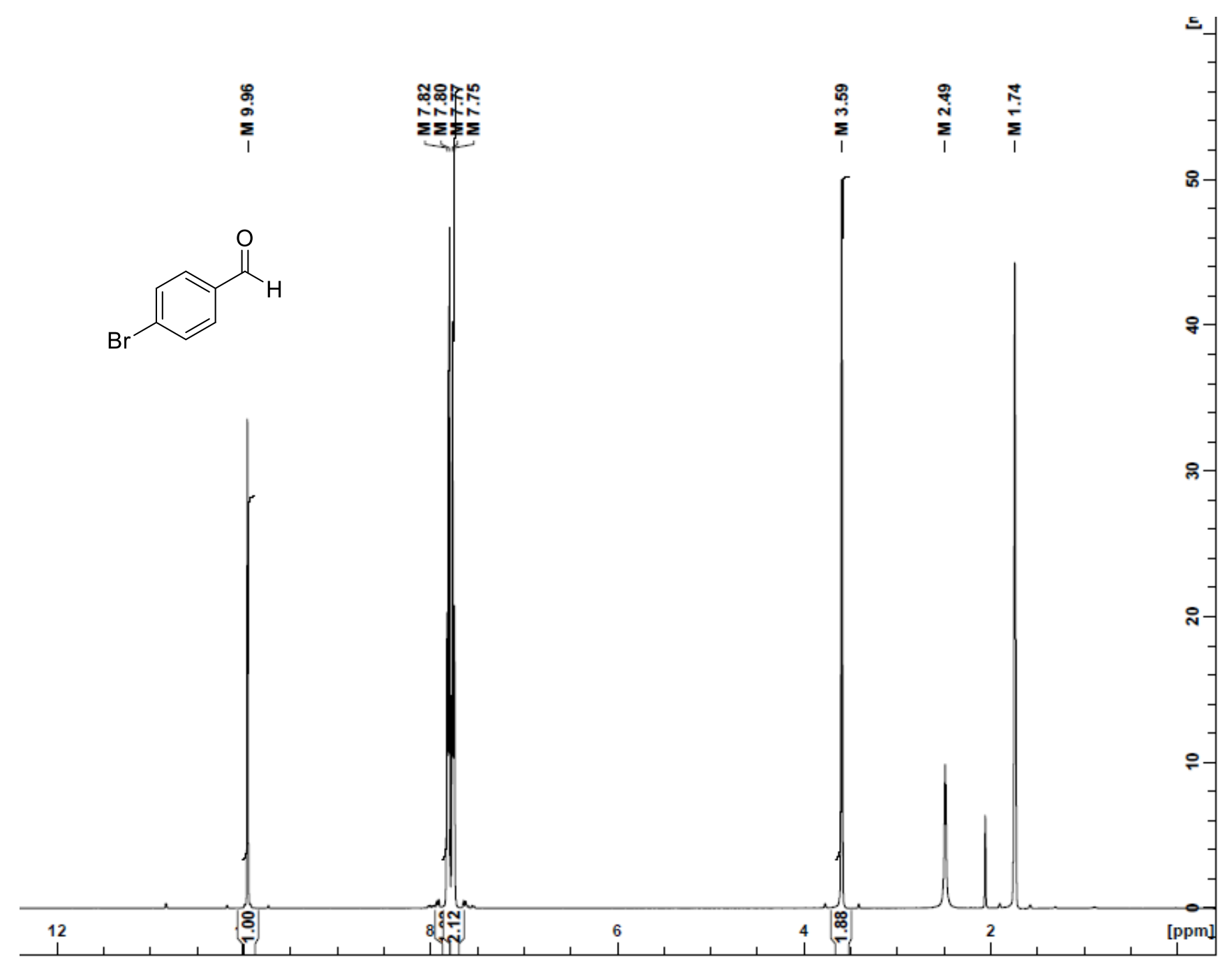




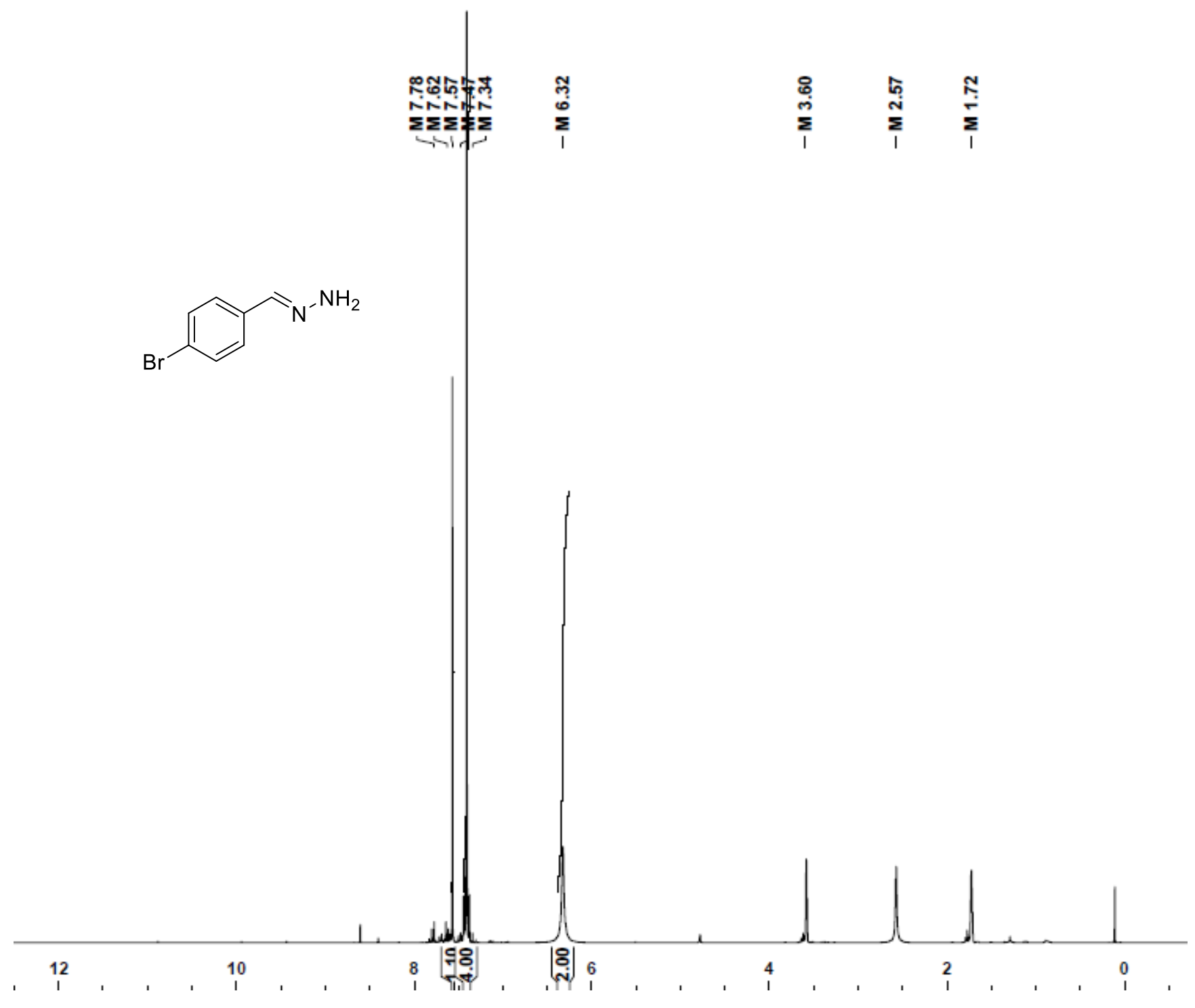




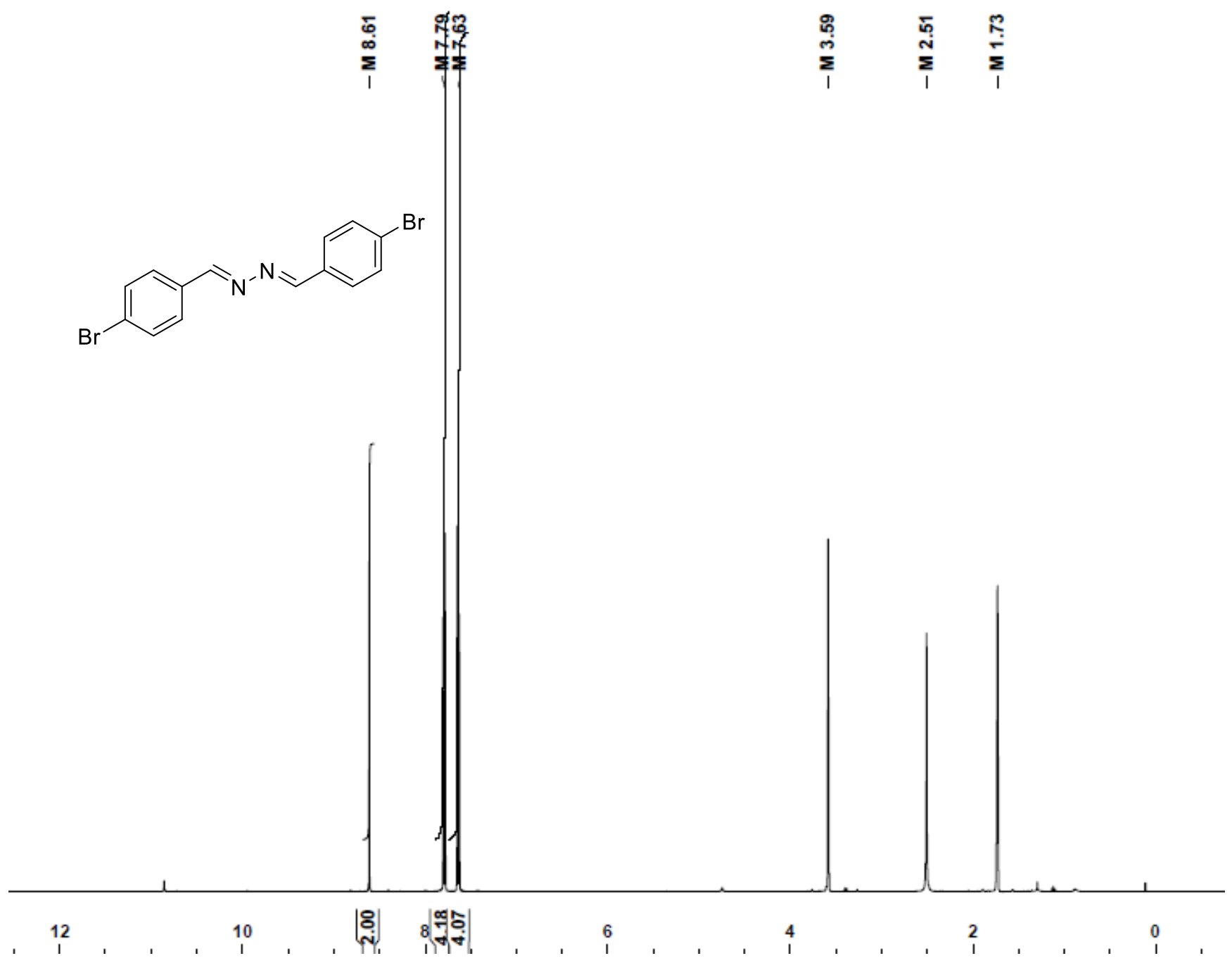




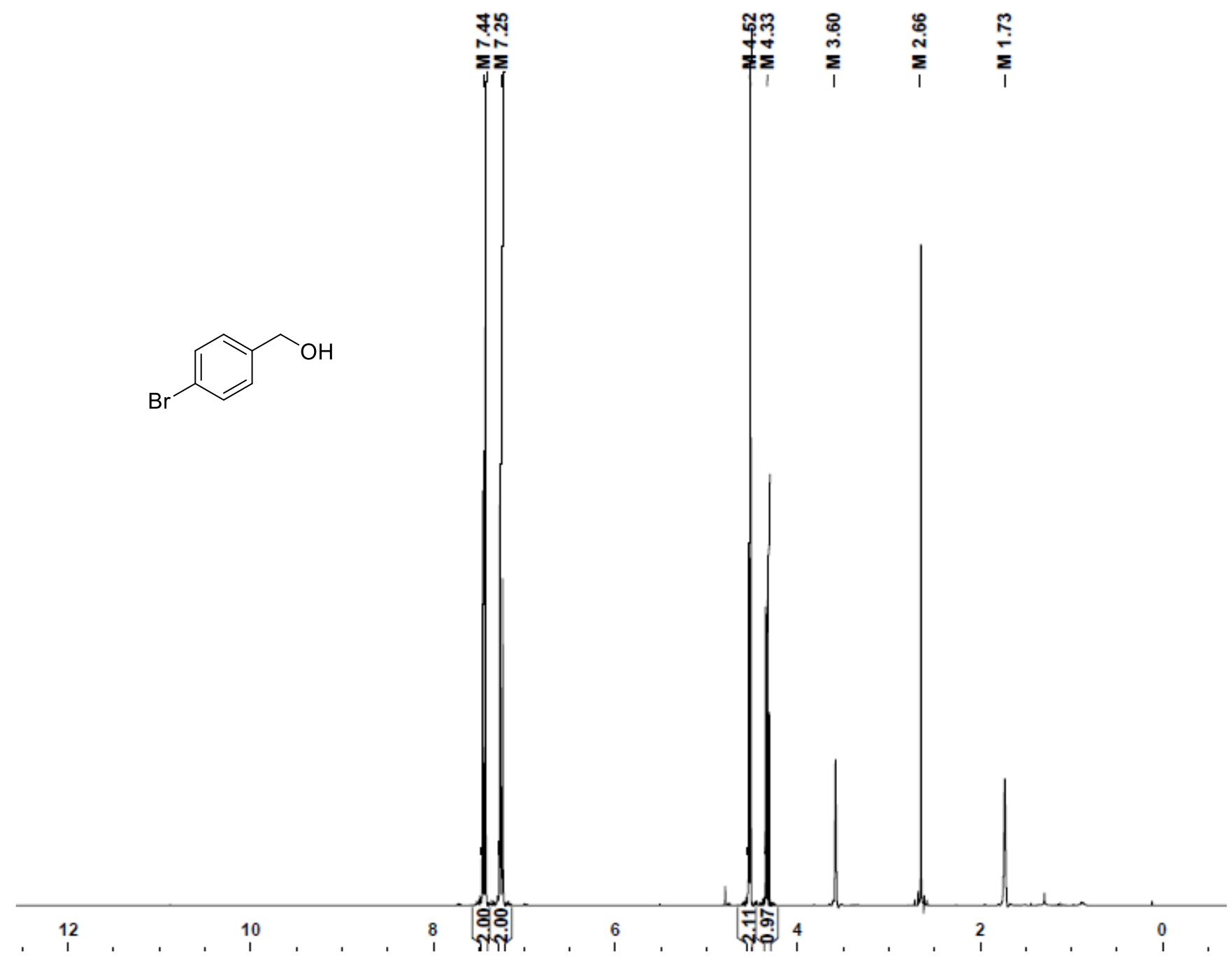




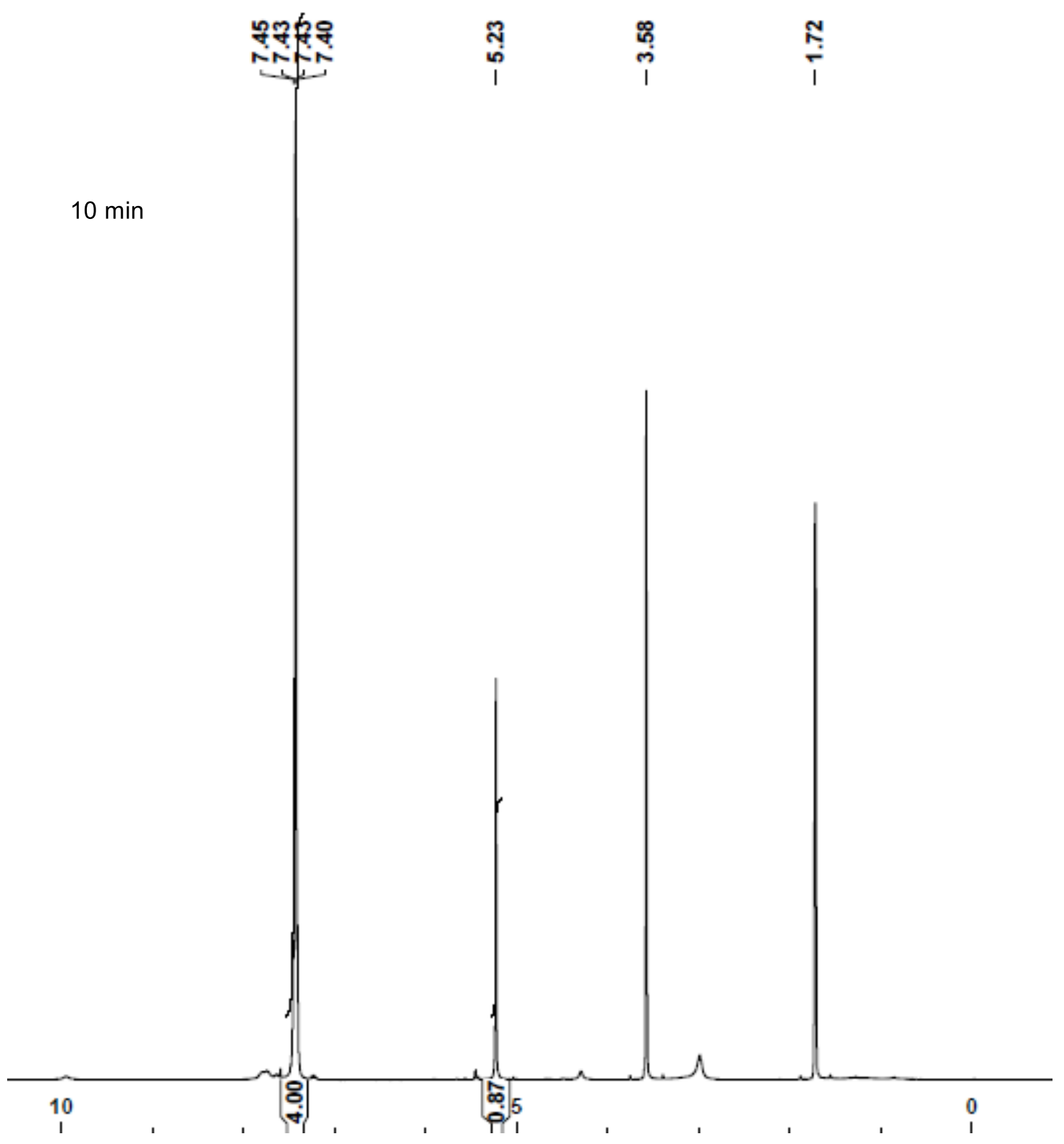




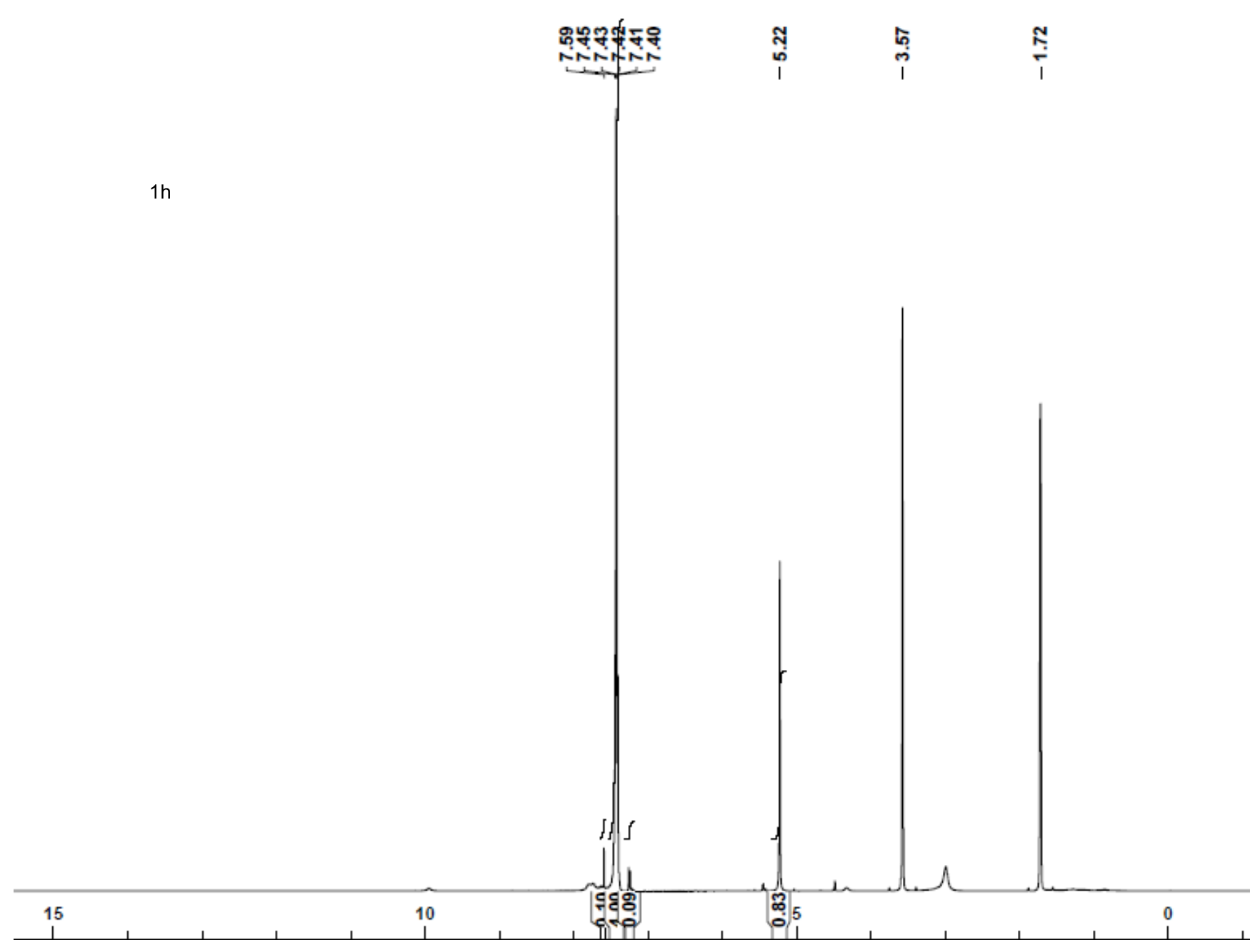




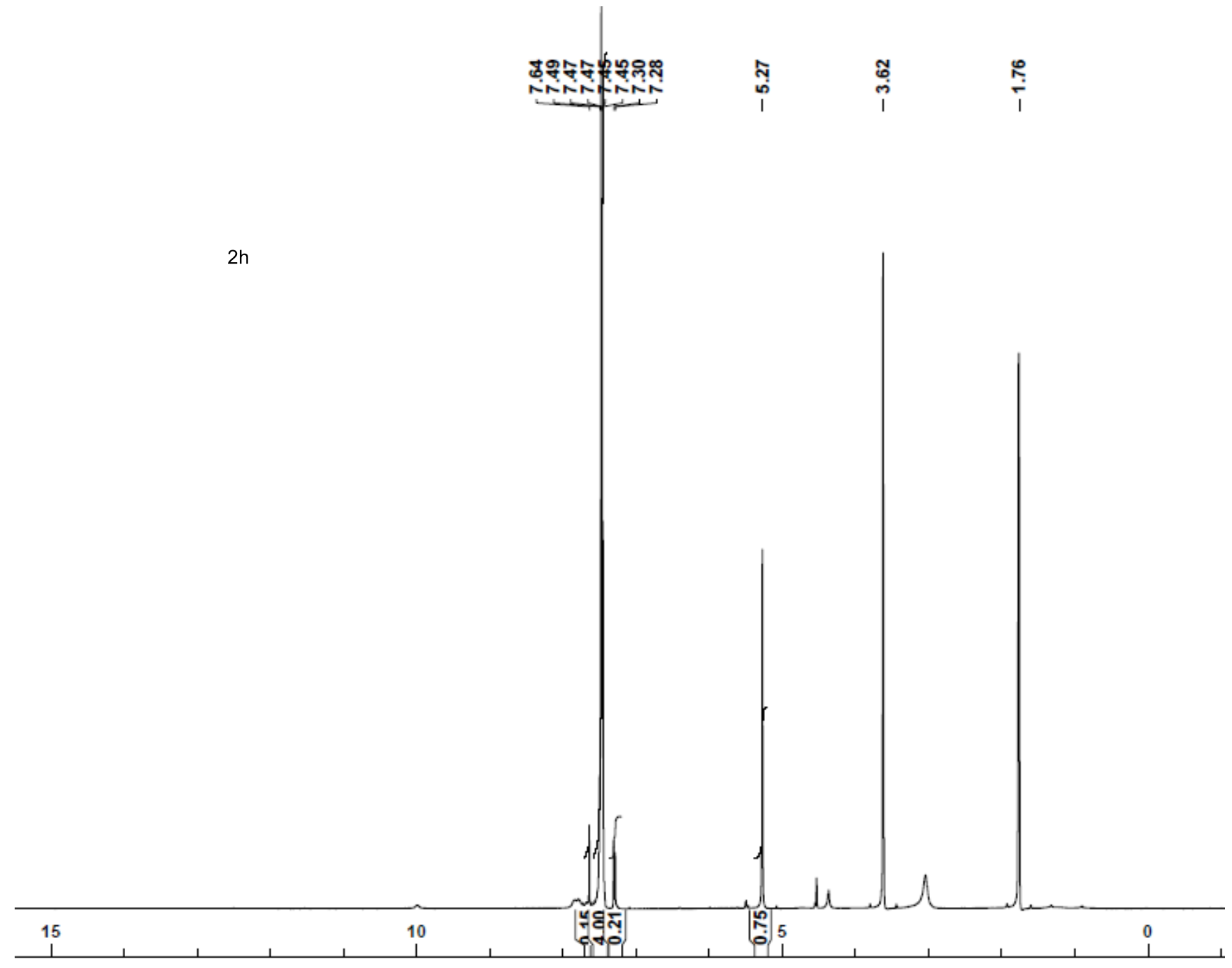




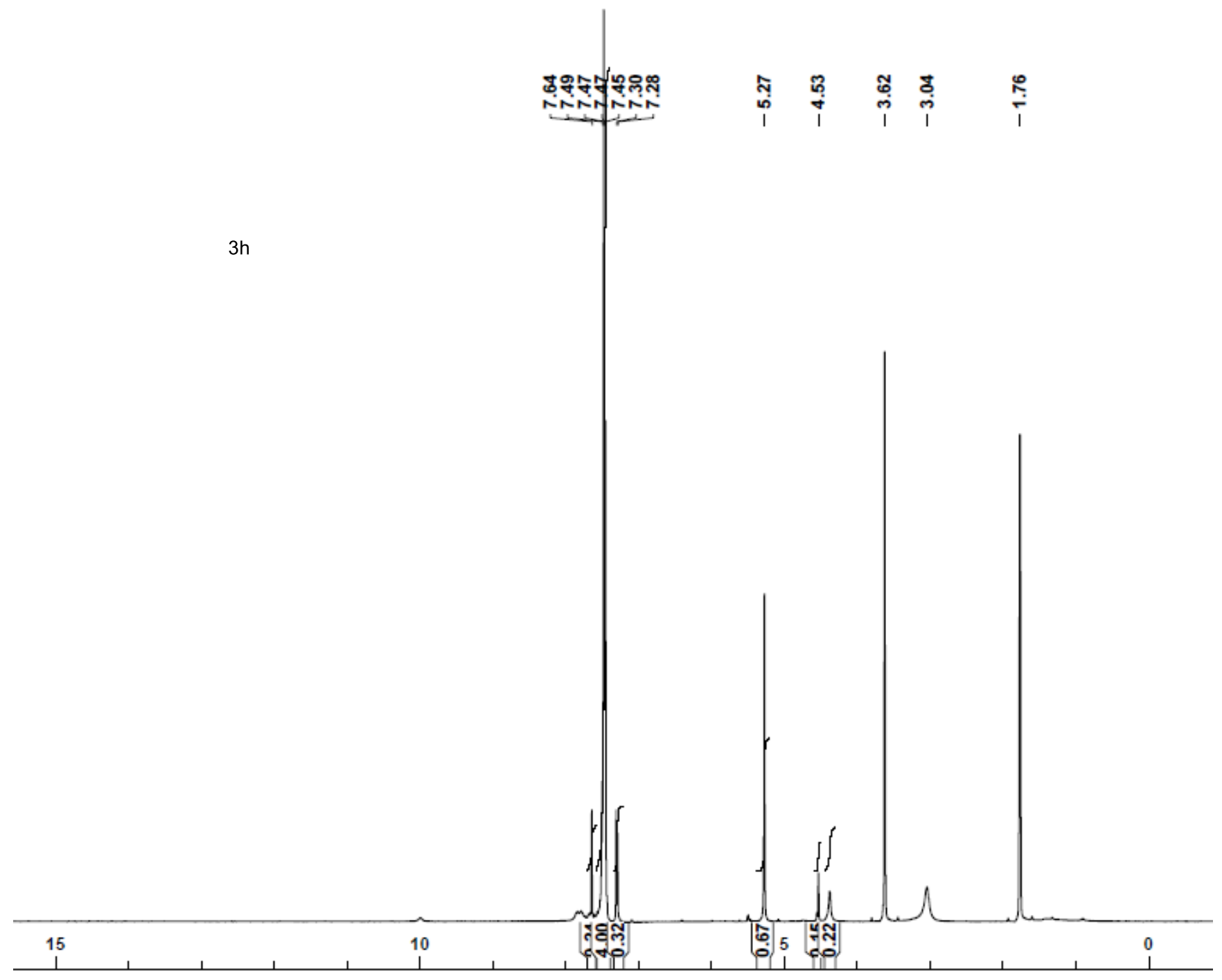





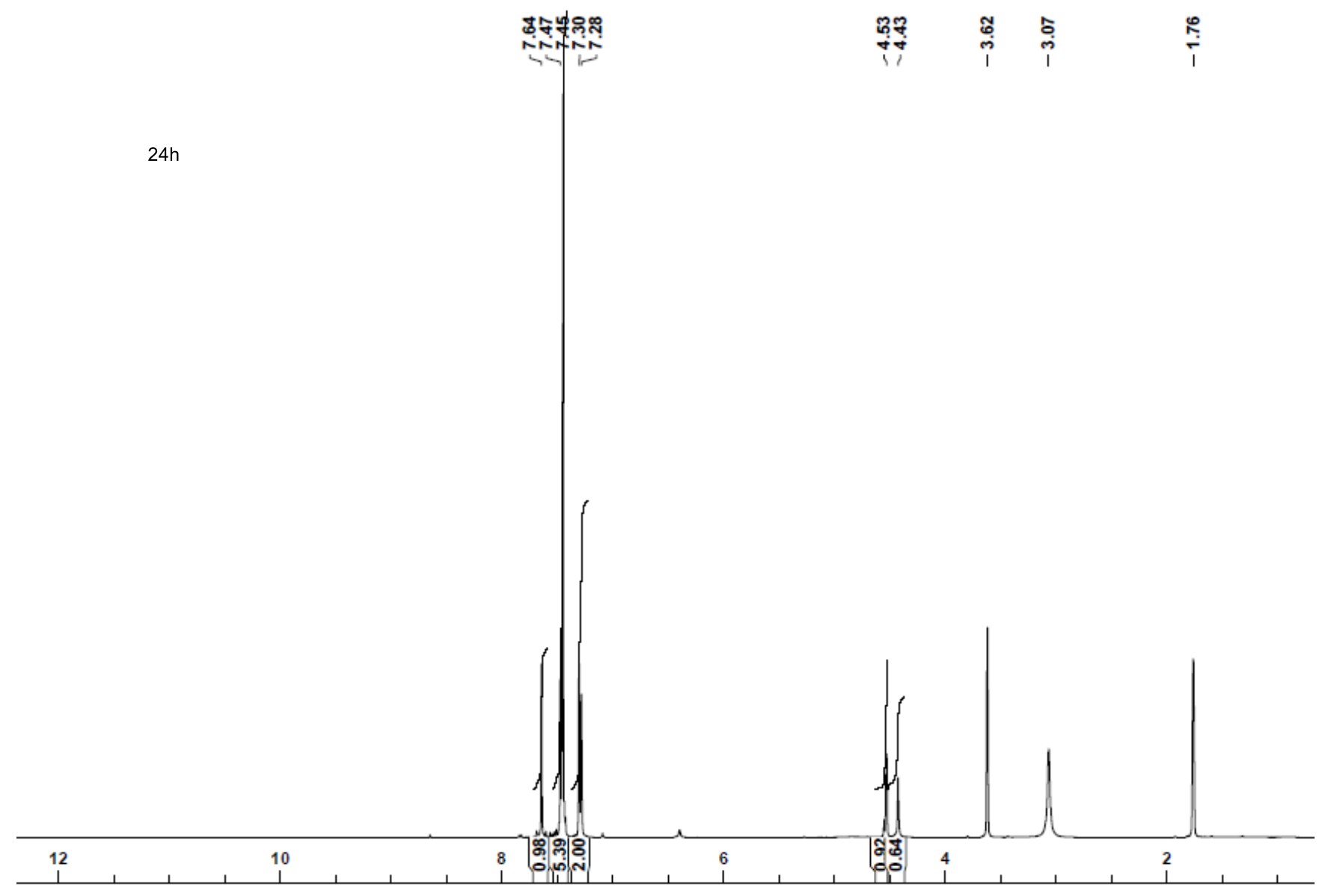




\section{GC/MS response to equimolar concentrations of $2 \mathrm{a}, 3 \mathrm{a}$ and $4 \mathrm{a}$.}

Formation of the product was also confirmed by GC-MS, but a quantification of the products (relative areas) is not possible by this method because of the high sensitivity of the detector to the azine.

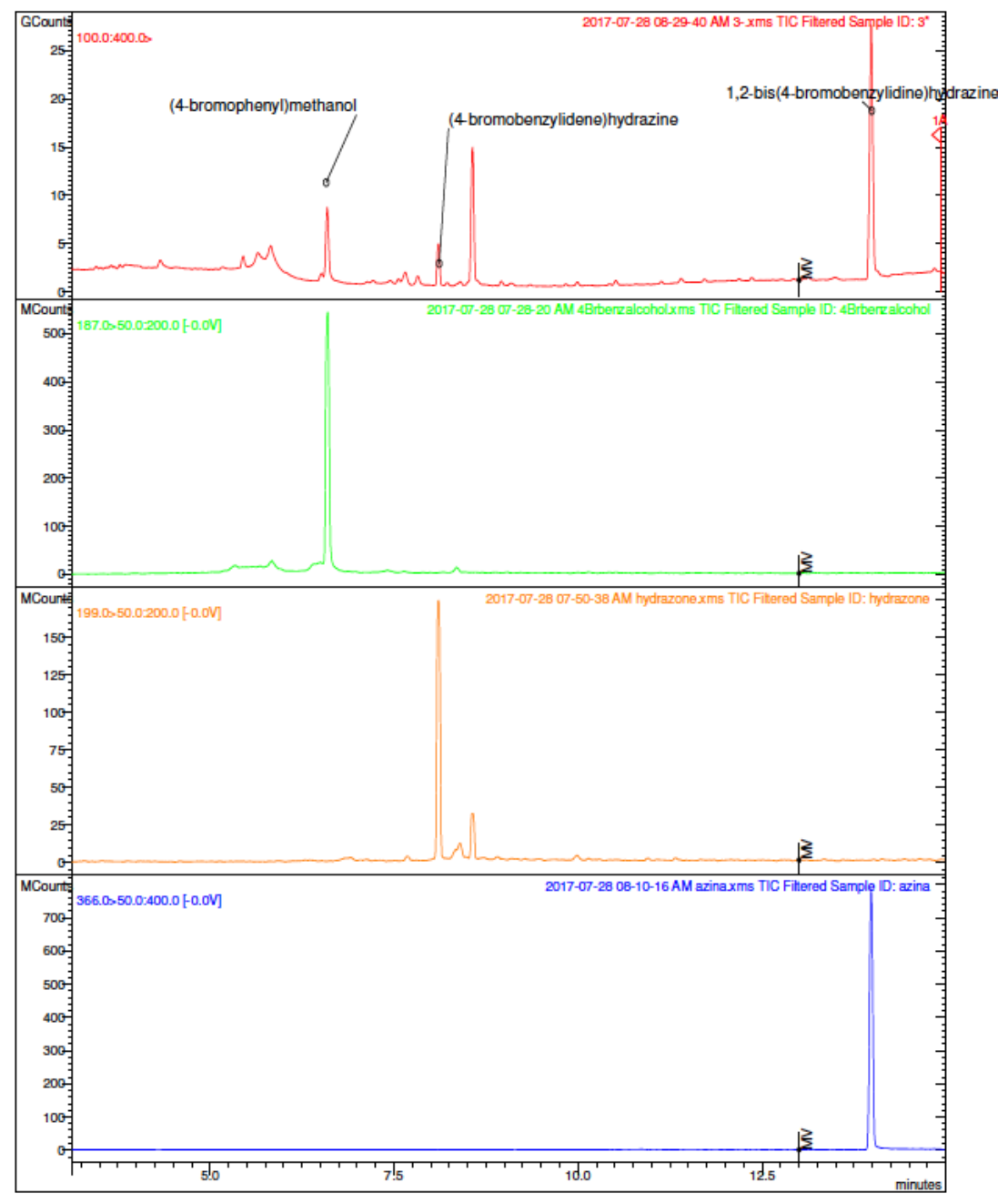




\section{Computational methods and models}

Computational details. Full optimization of all stationary structures were carried out using the hybrid metaGGA M06-2X functional in conjunction with the $6-31+\mathrm{G}(\mathrm{d}, \mathrm{p})$ basis set. ${ }^{27}$ This level of theory is well suited for computing activation barriers and to capture the nonbonding interactions relevant to reaction kinetics. All of the TS structures were optimized with $\mathrm{SMD}^{28}$ corrections to mimic solvation effects by THF used as reaction medium in the experimental study. Harmonic analysis and Intrinsic Reaction Coordinate (IRC) ${ }^{29}$ calculations were performed to confirm the nature of the proposed transition state geometries. 3D structures were generated by using CYLView program. ${ }^{30}$ All calculations were carried using Gaussian 09. ${ }^{31}$

\section{Reactant Complex-1a (hydrazine)}

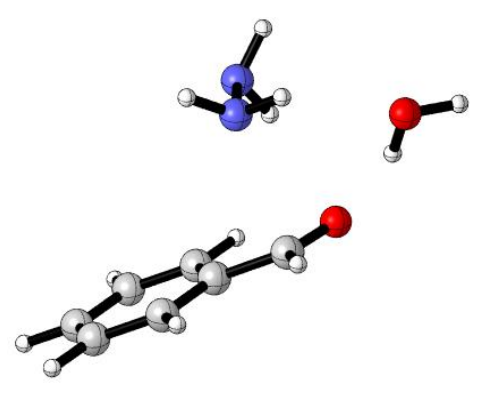

$\begin{array}{lrrr}\text { C } & 2.84685100 & -0.11267300 & -1.16331000 \\ \mathrm{C} & 1.53940200 & -0.59099900 & -1.19635600 \\ \mathrm{C} & 0.76893100 & -0.59421300 & -0.03015200 \\ \mathrm{C} & 1.30265200 & -0.11731600 & 1.17218000 \\ \mathrm{C} & 2.60746300 & 0.36231700 & 1.20248400 \\ \mathrm{C} & 3.37789600 & 0.36465200 & 0.03580700 \\ \mathrm{H} & 3.44859400 & -0.10764300 & -2.06673100 \\ \mathrm{H} & 1.10778600 & -0.95781400 & -2.12492600 \\ \mathrm{H} & 0.68975500 & -0.12639800 & 2.06901100 \\ \mathrm{H} & 3.02725100 & 0.73753300 & 2.13069200 \\ \mathrm{H} & 4.39641800 & 0.74075100 & 0.06315500 \\ \mathrm{C} & -0.62003500 & -1.09166800 & -0.09250800 \\ \mathrm{O} & -1.35601600 & -1.16250400 & 0.88287800 \\ \mathrm{H} & -0.96101500 & -1.44257300 & -1.08030400 \\ \mathrm{~N} & -1.72956600 & 1.83096500 & 0.38895400 \\ \mathrm{H} & -2.63237400 & 2.24936900 & 0.60565900 \\ \mathrm{H} & -1.59838800 & 1.06738200 & 1.05071500 \\ \mathrm{~N} & -1.73739300 & 1.25864000 & -0.92020800 \\ \mathrm{H} & -2.67045000 & 0.93635400 & -1.17486400 \\ \mathrm{H} & -1.44458100 & 1.97421200 & -1.57744400 \\ \mathrm{O} & -3.91896300 & -0.59190700 & -0.16451200 \\ \mathrm{H} & -4.68907300 & -1.04478900 & 0.19654900 \\ \mathrm{H} & -3.14434000 & -0.93892200 & 0.31146900 \\ \mathrm{H} & & \end{array}$

Energy: -533.6992526 a.u

TS-1a (nucleophilic addition of hydrazine; water as HB-donor) 


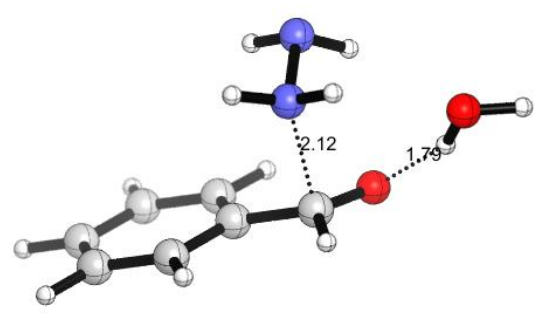

$\begin{array}{lrrr}\mathrm{C} & -0.00948400 & -0.04868300 & 0.00613300 \\ \mathrm{C} & 0.00292200 & -0.06321000 & 1.39865600 \\ \mathrm{C} & 1.21720400 & -0.03377200 & 2.08964000 \\ \mathrm{C} & 2.42127300 & 0.00568900 & 1.38335100 \\ \mathrm{C} & 2.40914800 & 0.02385100 & -0.01113700 \\ \mathrm{C} & 1.19546800 & -0.00347000 & -0.69941000 \\ \mathrm{H} & -0.95326600 & -0.07584700 & -0.53040600 \\ \mathrm{H} & -0.93033600 & -0.09564300 & 1.95785700 \\ \mathrm{H} & 3.35536800 & 0.01140900 & 1.93815900 \\ \mathrm{H} & 3.34484700 & 0.05399700 & -0.56174300 \\ \mathrm{H} & 1.18725200 & 0.00636700 & -1.78547800 \\ \mathrm{C} & 1.22628800 & -0.04499700 & 3.58491400 \\ \mathrm{O} & 2.28670800 & -0.18406900 & 4.22132100 \\ \mathrm{H} & 0.25481300 & -0.28017900 & 4.05020800 \\ \mathrm{~N} & 2.08587000 & 2.69524800 & 3.73723400 \\ \mathrm{H} & 2.72965000 & 2.12084100 & 4.28762400 \\ \mathrm{H} & 2.39442000 & 2.58239400 & 2.77012600 \\ \mathrm{~N} & 0.82321200 & 2.02189000 & 3.83035100 \\ \mathrm{H} & 0.48815700 & 2.15707900 & 4.78530700 \\ \mathrm{H} & 0.16961000 & 2.48263300 & 3.19825100 \\ \mathrm{O} & 1.55738700 & 1.16390700 & 6.46243000 \\ \mathrm{H} & 1.90676300 & 0.95736700 & 7.33591500 \\ \mathrm{H} & 1.92355200 & 0.50245600 & 5.83877200\end{array}$

Energy: -533.6934859 a.u

Int-1a

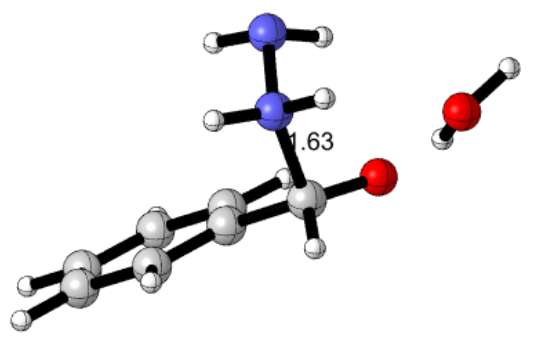

$$
\begin{array}{llll}
\text { C } & -2.78348500 & -1.10730100 & -0.26437000 \\
\text { C } & -1.41363300 & -1.18080700 & -0.51199900
\end{array}
$$

S15 
$-0.61148000$

$-1.19118900$

$-2.56216600$

$-3.35914600$

$-3.40234600$

$-0.96631800$

$-0.55169800$

$-3.01027900$

$-4.42696000$

0.88790700

1.52723800

1.09890400

1.49807100

1.80155400

0.52430000

1.48500300

2.46831100

1.00325500

3.86492200

4.58849400

3.05314400
$-0.04298500$

1.17239900

1.24897300

0.10981500

$-1.99405200$

$-2.12629200$

2.04804700

2. 19822700

0.17015000

$-0.11799300$

1.01913200

$-0.88582000$

$-0.08482600$

0.83359800

0.02862900

$-0.89583000$

$-1.10171900$

$-1.77597900$

$-0.03255400$

0.53406300

0.55050100
$-0.38867400$

$-0.02623500$

0.22261400

0.10541000

$-0.36729900$

$-0.81605600$

0.03893600

0.50249300

0.29466000

$-0.64022700$

$-0.73963900$

$-1.41325300$

1.84241800

1.50276900

2.12747300

0.66699100

0.44046400

0.86957300

$-0.59396400$

$-0.30681000$

$-0.74910600$

Energy: -533.6965176 a.u

TS-2a (formation of hemiaminal intermediate; water as proton shuttle)

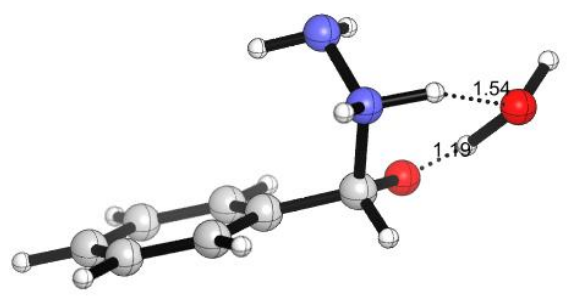

$\begin{array}{lr}\mathrm{C} & -0.06347600 \\ \mathrm{C} & -0.00639900 \\ \mathrm{C} & 1.22861500 \\ \mathrm{C} & 2.40681900 \\ \mathrm{C} & 2.35049300 \\ \mathrm{C} & 1.11765700 \\ \mathrm{H} & -1.02527100 \\ \mathrm{H} & -0.92642000 \\ \mathrm{H} & 3.35620700 \\ \mathrm{H} & 3.26997700 \\ \mathrm{H} & 1.07518100 \\ \mathrm{C} & 1.27925000 \\ \mathrm{O} & 2.51288800 \\ \mathrm{H} & 0.51319800 \\ \mathrm{~N} & 1.46196100 \\ \mathrm{H} & 2.44193700\end{array}$

$-0.06330000$

$-0.03197200$

0.00683400

0.01028000

$-0.02544500$

$-0.06028000$

$-0.09842000$

$-0.04665700$

0.03183700

$-0.02773800$

$-0.09030300$

0.07805600

$-0.07020900$

$-0.59001400$

2. 59868000

2.45628900
0.07163300

1.46340100

2.11738600

1.37135200

$-0.02294300$

$-0.67350200$

$-0.43152700$

2. 04579600

1.89750200

$-0.60100600$

$-1.75832900$

3. 62851900

4.15802900

4.06184800

3. 54294700

3. 79125000 
$\mathrm{H}$
$\mathrm{N}$
$\mathrm{H}$
$\mathrm{H}$
$\mathrm{O}$
$\mathrm{H}$
$\mathrm{H}$
Energy: $\mathbf{- 5 3 3 . 6 9 0 6 0 2 4} \mathbf{a . u}$

Int-2a

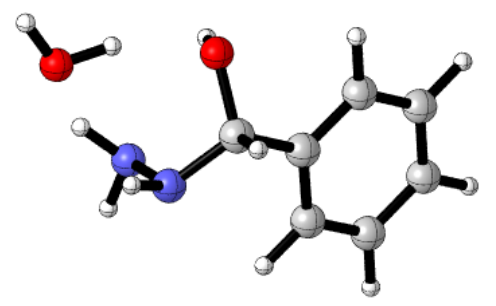

$\begin{array}{ll}\mathrm{C} & -2.70744800 \\ \mathrm{C} & -1.34699700 \\ \mathrm{C} & -0.68548100 \\ \mathrm{C} & -1.40992700 \\ \mathrm{C} & -2.77141400 \\ \mathrm{C} & -3.42410500 \\ \mathrm{H} & -3.20789000 \\ \mathrm{H} & -0.78860500 \\ \mathrm{H} & -0.90689600 \\ \mathrm{H} & -3.32110600 \\ \mathrm{H} & -4.48335600 \\ \mathrm{C} & 0.79034800 \\ \mathrm{O} & 1.39976100 \\ \mathrm{H} & 0.89899800 \\ \mathrm{~N} & 1.64295400 \\ \mathrm{H} & 1.54398700 \\ \mathrm{H} & 2.58289600 \\ \mathrm{~N} & 1.47794100 \\ \mathrm{H} & 4.82214300 \\ \mathrm{O} & 4.03708100 \\ \mathrm{H} & 3.27481000 \\ \mathrm{H} & 2.38236600 \\ \mathrm{H} & 1.19181500\end{array}$

Energy: -533.7151471 a.u
1.40528800

0.76128200

0.98424500

$-0.23908300$

1.85715900

2.46586100

2.36977300
2.54490700

1.47587100

1.44947500

1.59603400

0.84260600

1.37382900

0.28743800
2.52510700

4.07531800

5.14490700

3.90123300

6.25936100

6.78283800

5.28065800
1.17681800

1.09897400

$-0.13199200$

$-1.28501600$

$-1.20736800$

0.02424800

2.14076300

1.99547000

$-2.24743800$

$-2.11070500$

0.08561200

$-0.23625400$

$-1.31797000$

$-0.50207000$

1. 30496600

2.30715100

1. 04081500

1.01827700

$-0.92032400$

$-0.37099700$

$-0.97097900$

0.97908400

$-1.18480800$
0.05331600

0.34828100

0.32020200

0.00075000

$-0.29335800$

$-0.26957900$

0.07300000

0.59728400

$-0.01792700$

$-0.54156100$

$-0.50164200$

0.67729500

$-0.03813000$

1. 73435400

$-0.89129900$

$-1.02176000$

$-1.18537400$

0.49693800

$-0.20928800$

$-0.10524600$

$-0.02665200$

0.96291400

$-0.97725500$

\section{TS-3a (water departure; proton shuttle mechanism)}




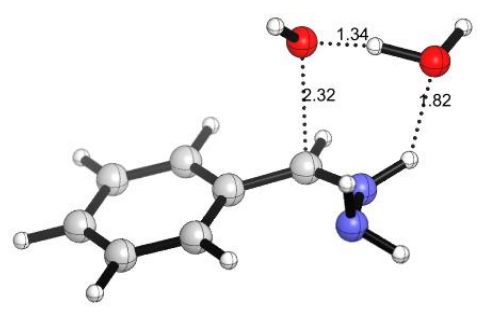
C
0.13725100
0.33014200
$-0.13199000$
0.02782700
0.37913500
1. 25533900
1.10611900
$-0.03800500$
2.05293900
2.28323200
$-0.49184900$
1.43518200
2.38746100
$-0.53134100$
0.05047700
1.31071700
$-0.12031700$
$-0.73653200$
$-0.70110100$
0.64849400
$-0.74384100$
$-0.88493200$
0.73117000
1.71632900
3.12109900
$-0.80112300$
2.05331700
3. 30417400
$-0.88134000$
$-0.41346300$
1.38674800
$-0.15007400$
$-1.81949100$
1.12041300
$-0.08354400$
3.51617700
2.61661200
1.59772800
4.06465800
1.97372700
$-0.56830400$
3.97397300
$-1.00039900$
0.87197600
4.00426300
$-1.71187200$
0.62472900
4.68810400
$-0.80589800$
1.86992500
4.11225400
0.16185900
0.17033400
4.35390900
2.30993500
1.07308600
6.95379200
1. 65236600
1.23017100
6.26916500
2.15607900
1.48584300
5.31683500
0.46228800
0.20355700
5.34469500
2.47579800
2.46725700
3.67307500

Energy: -533.6588336 a.u

\section{Hydrazone}
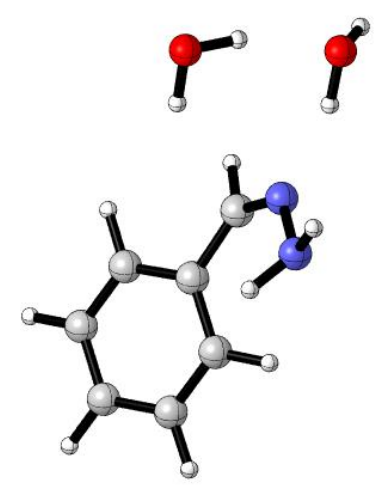

C
C
C
C
C
C
H

$-3.04973900$

$-0.98597000$

0.22380200

$-1.68484500$

$-1.08962800$

$-0.04001500$

$-0.93533900$

0.05914900

$-0.33866300$

$-1.58603800$

1.30159700

$-0.38586500$

$-2.94512500$

1.40296300

$-0.10655900$

$-3.68029400$

0.25729000

0.20263500

$-3.62126300$

$-1.88236000$

0.44498200 
$-1.21546800$

$-1.01545200$

$-3.43263100$

$-4.74229000$

0.50512000

3.30000000

0.85475600

1.15386600

1.99928500

0.38494400

1.42878100

4. 31459500

4.11699700

3.74140700

3.18455800

2.39061600
$-2.06817800$

2.19076000

2. 37270000

0.33251500

0.03445800

1. 61588200

0.79742200

$-1.74504200$

$-1.98388000$

$-1.55063600$

$-0.73864400$

0.01447300

$-0.19238300$

1.10330500

$-0.50182900$

1.29437000
$-0.04748800$

$-0.64255900$

$-0.13746500$

0.41642200

$-0.66885300$

1.22308900

$-1.36398600$

0.66098800

1.16714100

1.30217800

$-0.22684500$

$-1.73405400$

$-0.81321800$

0.52324000

$-0.77910300$

1.21383500

Energy: -533.7121627 a.u.

\section{Reactant complex 2a (diimide)}
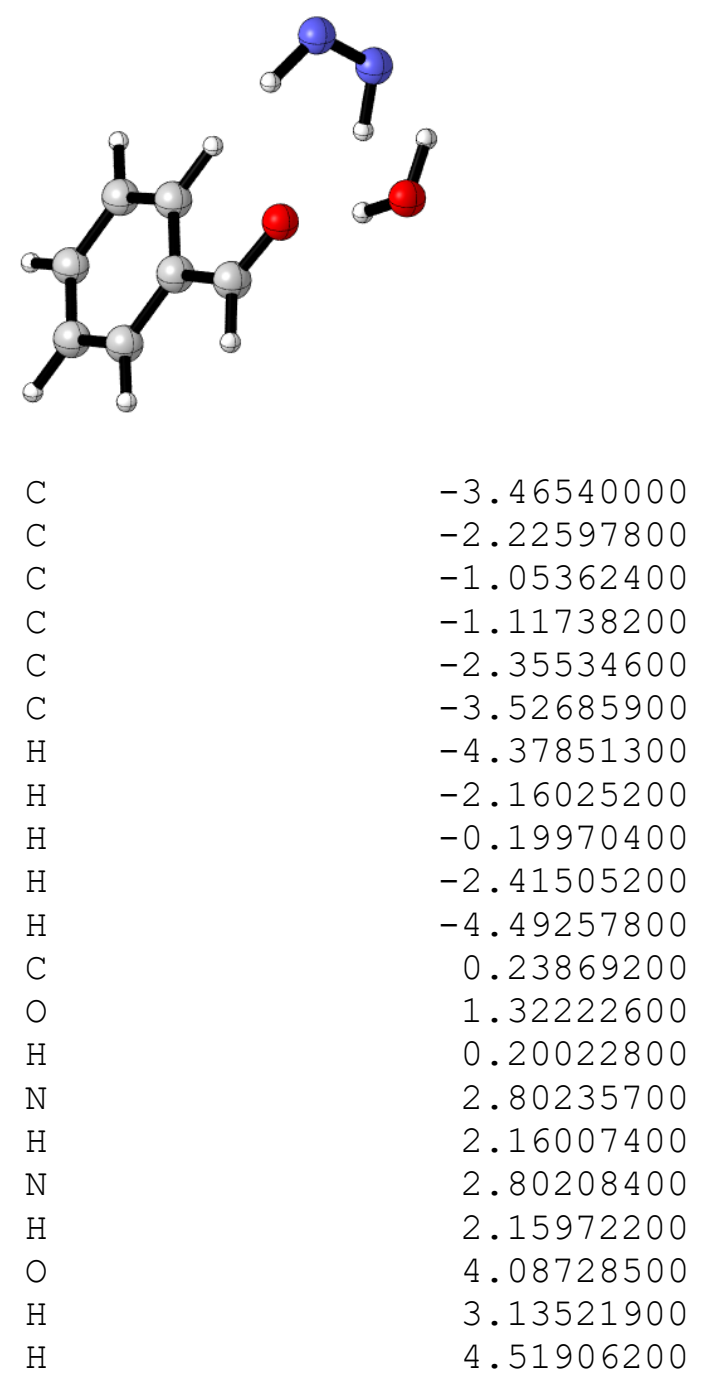

$$
\begin{array}{r}
-0.70442000 \\
-1.33902000 \\
-0.57508300 \\
0.82533300 \\
1.45425300 \\
0.68900900 \\
-1.29082200 \\
-2.42455600 \\
1.40748900 \\
2.53796900 \\
1.18600200 \\
-1.27245500 \\
-0.70212700 \\
-2.37469400 \\
1.74995900 \\
1.02640200 \\
1.74885600 \\
1.02454400 \\
-1.54788700 \\
-1.36413300 \\
-0.68549100
\end{array}
$$$$
0.00099200
$$$$
0.00031800
$$

$-0.00053500$

$-0.00071400$

0.00000100

0.00085500

0.00161300

0.00040100

$-0.00154000$

$-0.00010500$

0.00140200

$-0.00134900$

$-0.00223900$

$-0.00121400$

$-0.61719300$

$-0.99868400$

0.61812800

0.99806000

0.00180300

$-0.00122100$

0.00082800

Energy: -532.4633419 a.u 


\section{TS-4a (reduction)}

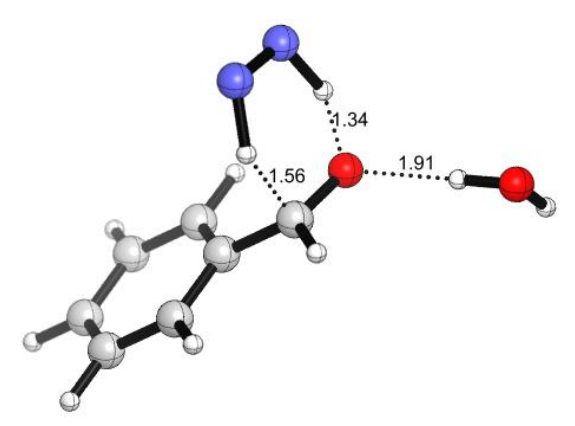

$\begin{array}{lrrr}\mathrm{C} & -0.02001600 & -0.26250300 & 0.15738300 \\ \mathrm{C} & 0.13970500 & -0.21401500 & 1.53925100 \\ \mathrm{C} & 1.40046600 & 0.04722600 & 2.08788800 \\ \mathrm{C} & 2.49948000 & 0.26767600 & 1.24940800 \\ \mathrm{C} & 2.33372400 & 0.22295500 & -0.13165700 \\ \mathrm{C} & 1.07628700 & -0.04240000 & -0.67831800 \\ \mathrm{H} & -0.99678800 & -0.47016300 & -0.26862400 \\ \mathrm{H} & -0.70903800 & -0.38218600 & 2.19828700 \\ \mathrm{H} & 3.47524000 & 0.46435900 & 1.68473900 \\ \mathrm{H} & 3.18452700 & 0.39374600 & -0.78416500 \\ \mathrm{H} & 0.95066000 & -0.07812500 & -1.75627600 \\ \mathrm{C} & 1.55336100 & 0.10869100 & 3.55866200 \\ \mathrm{O} & 2.71079700 & 0.14175600 & 4.09906800 \\ \mathrm{H} & 0.69792900 & -0.21051400 & 4.17071000 \\ \mathrm{~N} & 2.72356800 & 2.55046900 & 4.34001000 \\ \mathrm{H} & 3.03666900 & 1.42440700 & 4.32891500 \\ \mathrm{~N} & 1.60201100 & 2.64244200 & 3.98481500 \\ \mathrm{H} & 1.13938400 & 1.60055900 & 3.73625200 \\ \mathrm{O} & 2.80282400 & -1.13242600 & 6.67896500 \\ \mathrm{H} & 2.77168200 & -0.70202800 & 5.81008300 \\ \mathrm{H} & 3.36517600 & -1.90602700 & 6.55913800\end{array}$

Energy: -532.4433366 a.u 
Prod 4 (alcohol)

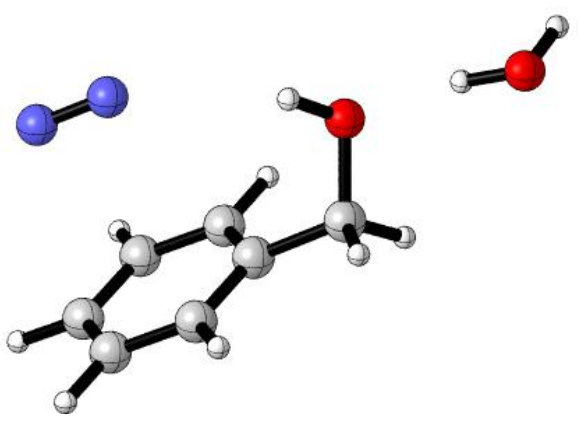

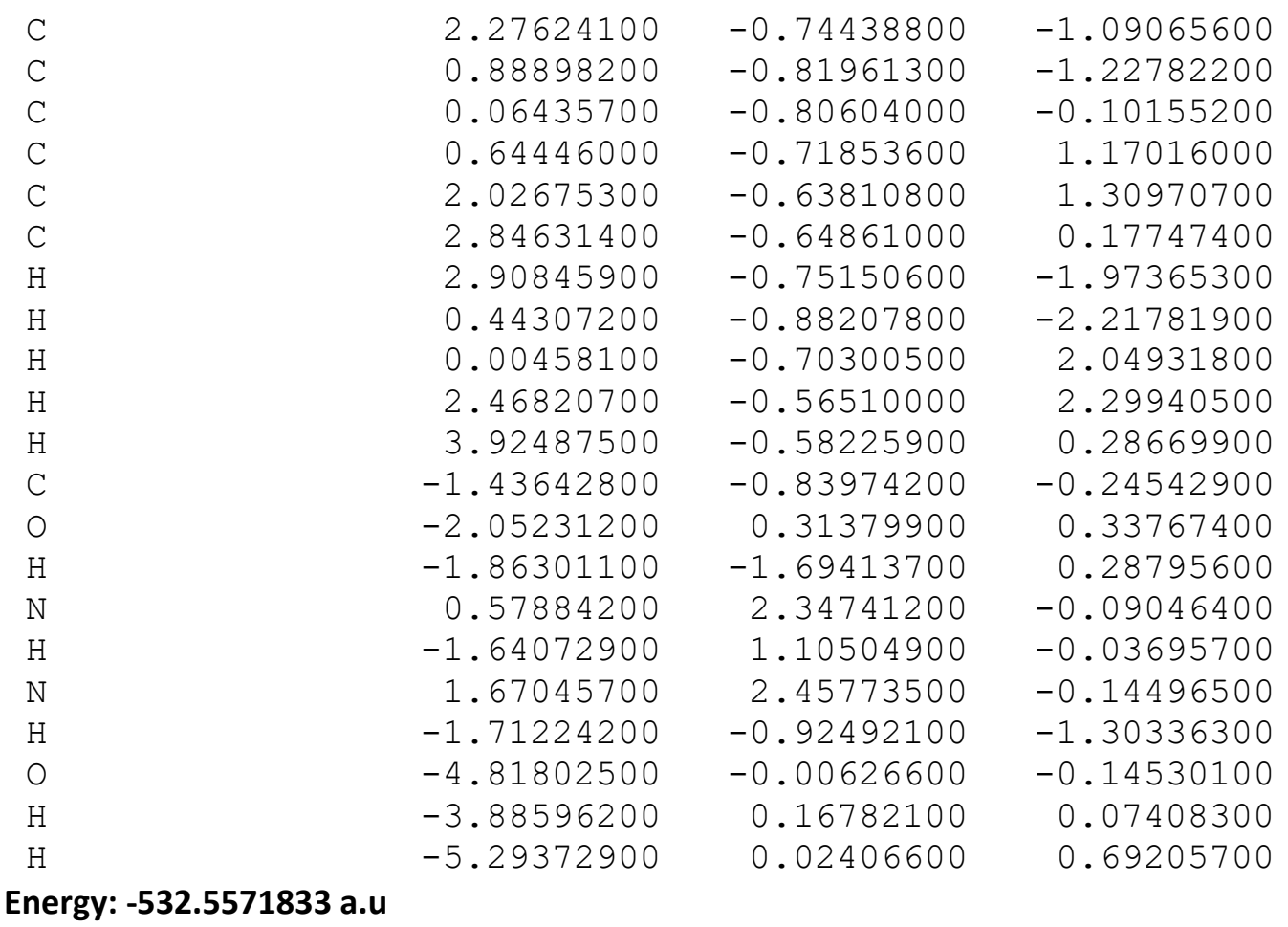

Reactant complex ib

S21 

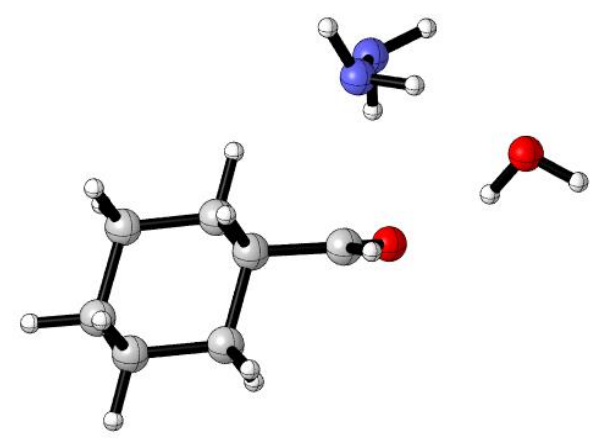

$\begin{array}{lrrr}\mathrm{C} & 0.68089400 & -0.93592300 & 0.08152700 \\ \mathrm{O} & 1.24759200 & -0.88347400 & -1.00007100 \\ \mathrm{H} & 1.10949800 & -1.55071000 & 0.89488300 \\ \mathrm{~N} & 2.51184500 & 1.73392700 & -0.14093800 \\ \mathrm{H} & 3.50783300 & 1.73497400 & -0.35329900 \\ \mathrm{H} & 2.06170400 & 1.20069800 & -0.88334400 \\ \mathrm{~N} & 2.26245000 & 1.03827200 & 1.08420500 \\ \mathrm{H} & 2.99741200 & 0.35310100 & 1.26097900 \\ \mathrm{H} & 2.27350500 & 1.72036300 & 1.83605700 \\ \mathrm{O} & 3.93504800 & -1.05746600 & -0.17722100 \\ \mathrm{H} & 4.43725200 & -1.86564500 & -0.33031500 \\ \mathrm{H} & 3.08650400 & -1.16353000 & -0.64309200 \\ \mathrm{C} & -0.64922800 & -0.30705800 & 0.36884900 \\ \mathrm{C} & -0.97568500 & 0.86534900 & -0.55392300 \\ \mathrm{C} & -1.72469100 & -1.41204500 & 0.27154600 \\ \mathrm{H} & -0.61481900 & 0.03710400 & 1.41230900 \\ \mathrm{C} & -2.36595100 & 1.42814700 & -0.24778700 \\ \mathrm{H} & -0.93766000 & 0.52115700 & -1.59574900 \\ \mathrm{H} & -0.21043200 & 1.64259600 & -0.44114500 \\ \mathrm{C} & -3.10982300 & -0.83867500 & 0.58038900 \\ \mathrm{H} & -1.71694600 & -1.82135500 & -0.74837700 \\ \mathrm{H} & -1.48709100 & -2.23542500 & 0.95561400 \\ \mathrm{C} & -3.43895700 & 0.34021200 & -0.33804900 \\ \mathrm{H} & -2.59585800 & 2.24830200 & -0.93679200 \\ \mathrm{H} & -2.36797500 & 1.85330100 & 0.76583800 \\ \mathrm{H} & -3.86630800 & -1.62486200 & 0.47879900 \\ \mathrm{H} & -3.13382400 & -0.50260200 & 1.62642700 \\ \mathrm{H} & -4.42129500 & 0.75367500 & -0.08361200 \\ \mathrm{H} & -3.50204300 & -0.01905800 & -1.37501000 \\ \mathrm{H} & & & \end{array}$

\section{Energy: -537.311666767 a.u}

\section{TS-1b}




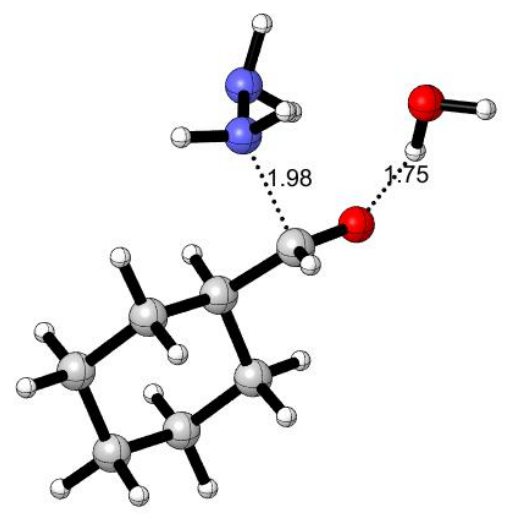

$\begin{array}{rrrr}\mathrm{C} & -0.37700100 & -0.03216200 & 0.35019800 \\ \mathrm{O} & -0.61672300 & -0.30902400 & 1.55468500 \\ \mathrm{H} & 0.63439100 & 0.32469900 & 0.07420500 \\ \mathrm{~N} & -1.01815900 & -2.65705700 & 0.13166500 \\ \mathrm{H} & -0.58742300 & -3.54679700 & 0.37271800 \\ \mathrm{H} & -1.27499500 & -2.20289500 & 1.01406300 \\ \mathrm{~N} & -0.04510800 & -1.80478200 & -0.45566000 \\ \mathrm{H} & 0.90195400 & -2.02608300 & -0.14067100 \\ \mathrm{H} & -0.10130200 & -1.86776200 & -1.46858100 \\ \mathrm{O} & 1.51384900 & -1.90101200 & 1.95766000 \\ \mathrm{H} & 2.17743600 & -1.66307100 & 2.61381100 \\ \mathrm{H} & 0.77816800 & -1.24943000 & 2.03659500 \\ \mathrm{C} & -1.48720600 & 0.50993700 & -0.53808900 \\ \mathrm{C} & -1.98534500 & 1.82009400 & 0.09735700 \\ \mathrm{C} & -1.03760700 & 0.75994200 & -1.98031600 \\ \mathrm{H} & -2.31306800 & -0.21691000 & -0.52403400 \\ \mathrm{C} & -3.09123800 & 2.46922600 & -0.73671900 \\ \mathrm{H} & -1.13653000 & 2.51655000 & 0.17990200 \\ \mathrm{H} & -2.33264500 & 1.61625900 & 1.11471900 \\ \mathrm{C} & -2.14406100 & 1.41168100 & -2.81496500 \\ \mathrm{H} & -0.15970500 & 1.42330000 & -1.96620900 \\ \mathrm{H} & -0.72257000 & -0.17310300 & -2.46009400 \\ \mathrm{C} & -2.63312100 & 2.71179600 & -2.17563000 \\ \mathrm{H} & -3.40536000 & 3.41032500 & -0.27114400 \\ \mathrm{H} & -3.96939900 & 1.80837900 & -0.74419900 \\ \mathrm{H} & -1.78199900 & 1.59767700 & -3.83246300 \\ \mathrm{H} & -2.98795700 & 0.71263900 & -2.89934600 \\ \mathrm{H} & -3.44735800 & 3.14430500 & -2.76821200 \\ \mathrm{H} & -1.81355500 & 3.44496200 & -2.17506900 \\ \mathrm{En} & & \end{array}$

Energy: -537.3070006 a.u

\section{Int-1b}




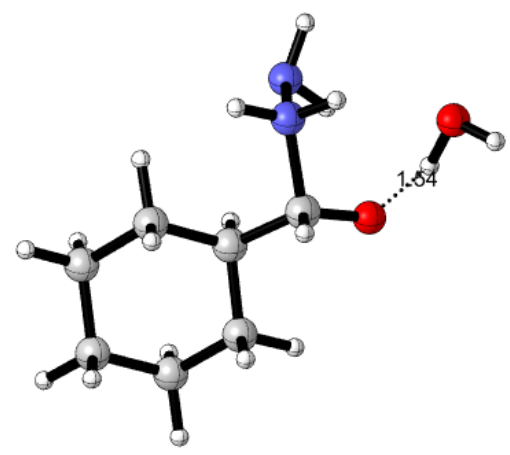

$\begin{array}{rrrr}\mathrm{C} & -0.95760200 & -0.36403700 & -0.29165100 \\ \mathrm{O} & -1.65022500 & -1.20027900 & 0.45234600 \\ \mathrm{H} & -0.94386300 & -0.57359500 & -1.38443200 \\ \mathrm{~N} & -1.93976300 & 1.50126300 & 1.02612500 \\ \mathrm{H} & -2.80611600 & 2.03514100 & 1.06316600 \\ \mathrm{H} & -2.09032600 & 0.64233800 & 1.56506700 \\ \mathrm{~N} & -1.77107200 & 1.03501000 & -0.31460000 \\ \mathrm{H} & -2.67442300 & 0.79614300 & -0.75188100 \\ \mathrm{H} & -1.29573400 & 1.74909500 & -0.86961900 \\ \mathrm{O} & -3.95594500 & -0.70498900 & -0.46967700 \\ \mathrm{H} & -4.36215700 & -1.37029000 & -1.03467500 \\ \mathrm{H} & -3.09510500 & -1.09605800 & -0.07834600 \\ \mathrm{C} & 0.46929000 & -0.07587100 & 0.19206600 \\ \mathrm{C} & 1.28091700 & -1.36782600 & 0.02145100 \\ \mathrm{C} & 1.15454700 & 1.09214700 & -0.52611800 \\ \mathrm{H} & 0.40725200 & 0.15318500 & 1.26591500 \\ \mathrm{C} & 2.73500100 & -1.19889000 & 0.46340000 \\ \mathrm{H} & 1.26091900 & -1.65678400 & -1.04149200 \\ \mathrm{H} & 0.79322100 & -2.17185100 & 0.58083500 \\ \mathrm{C} & 2.61568000 & 1.25025700 & -0.09286000 \\ \mathrm{H} & 1.11266400 & 0.92350800 & -1.61373100 \\ \mathrm{H} & 0.63016300 & 2.03441200 & -0.31996300 \\ \mathrm{C} & 3.40615400 & -0.04454900 & -0.28201200 \\ \mathrm{H} & 3.28509700 & -2.13274800 & 0.29976600 \\ \mathrm{H} & 2.76415300 & -0.99638600 & 1.54335300 \\ \mathrm{H} & 3.08245500 & 2.07022400 & -0.65026500 \\ \mathrm{H} & 2.64028700 & 1.53329300 & 0.96896400 \\ \mathrm{H} & 4.43783400 & 0.08740000 & 0.06323600 \\ \mathrm{H} & 3.45495500 & -0.28618000 & -1.35358500 \\ \mathrm{En} & & \end{array}$

Energy: -537.309743824 a.u

\section{TS-2b}




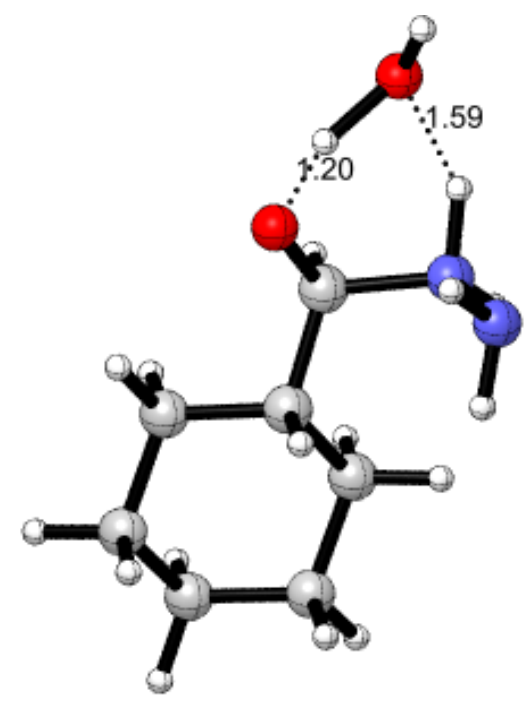

$\begin{array}{lrrr}\mathrm{C} & -0.09438300 & 0.06699800 & 0.18427700 \\ \mathrm{O} & -0.37962400 & -0.08198000 & 1.49904900 \\ \mathrm{H} & 0.98179700 & 0.22857200 & -0.02594600 \\ \mathrm{~N} & -1.66281300 & -1.81396000 & -0.47961800 \\ \mathrm{H} & -1.98045900 & -1.75072300 & 0.48873800 \\ \mathrm{H} & -2.23511300 & -1.16714900 & -1.02156800 \\ \mathrm{~N} & -0.32180000 & -1.32086400 & -0.47528600 \\ \mathrm{H} & 0.23144700 & -1.96179900 & 0.19467300 \\ \mathrm{H} & 0.05727700 & -1.37922000 & -1.42258500 \\ \mathrm{O} & 0.81252200 & -2.14055300 & 1.66000000 \\ \mathrm{H} & 0.50619400 & -2.83357500 & 2.25366800 \\ \mathrm{H} & 0.21793600 & -1.07992000 & 1.79175200 \\ \mathrm{C} & -0.91088000 & 1.15290000 & -0.50770400 \\ \mathrm{C} & -0.55997700 & 2.50530000 & 0.12712500 \\ \mathrm{C} & -0.68306700 & 1.19272200 & -2.02473100 \\ \mathrm{H} & -1.97495600 & 0.95367500 & -0.30568700 \\ \mathrm{C} & -1.31898900 & 3.65788400 & -0.53240500 \\ \mathrm{H} & 0.52216800 & 2.67426700 & 0.01579300 \\ \mathrm{H} & -0.76910700 & 2.46275000 & 1.20004100 \\ \mathrm{C} & -1.44025300 & 2.35095600 & -2.68117400 \\ \mathrm{H} & 0.39406200 & 1.30626400 & -2.22036300 \\ \mathrm{H} & -0.99385300 & 0.25105200 & -2.49800700 \\ \mathrm{C} & -1.07470600 & 3.69096000 & -2.04207300 \\ \mathrm{H} & -1.02067800 & 4.60843100 & -0.07612100 \\ \mathrm{H} & -2.39507600 & 3.53762000 & -0.34280600 \\ \mathrm{H} & -1.23116600 & 2.36893800 & -3.75653800 \\ \mathrm{H} & -2.52055400 & 2.18131000 & -2.56930900 \\ \mathrm{H} & -1.64875800 & 4.50113800 & -2.50566400 \\ \mathrm{H} & -0.01264800 & 3.90250500 & -2.23133300 \\ \mathrm{En} & & \end{array}$

Energy: -537.3048447 a.u

Int-2b 


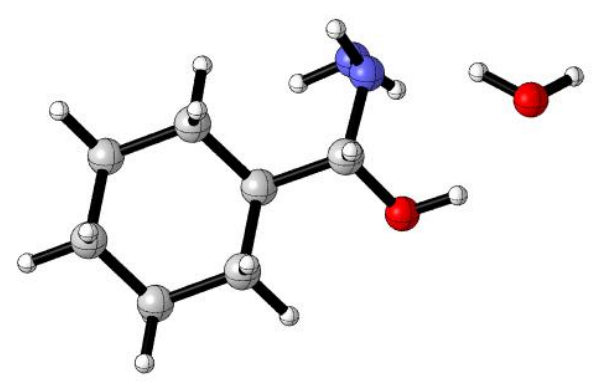

$\begin{array}{rrrr}\mathrm{C} & -0.97808200 & -0.28959200 & -0.25346100 \\ \mathrm{O} & -1.54972600 & -1.27140100 & 0.57673700 \\ \mathrm{H} & -0.97455600 & -0.61481400 & -1.30710000 \\ \mathrm{~N} & -1.86951300 & 1.65471500 & 0.96652300 \\ \mathrm{H} & -2.41886100 & 1.12552100 & 1.63881500 \\ \mathrm{H} & -0.93434500 & 1.75763700 & 1.36281400 \\ \mathrm{~N} & -1.83725700 & 0.90633200 & -0.24396800 \\ \mathrm{H} & -3.47019300 & 0.10610300 & -0.58154700 \\ \mathrm{H} & -1.57146800 & 1.53512100 & -0.99757900 \\ \mathrm{O} & -4.05284100 & -0.68670800 & -0.66443900 \\ \mathrm{H} & -4.90898100 & -0.45856800 & -0.28464800 \\ \mathrm{H} & -2.45753200 & -1.43164200 & 0.26726300 \\ \mathrm{C} & 0.46746300 & -0.07919900 & 0.19909900 \\ \mathrm{C} & 1.27429300 & -1.37129100 & 0.01323200 \\ \mathrm{C} & 1.13279100 & 1.08100000 & -0.55249800 \\ \mathrm{H} & 0.45495500 & 0.15355500 & 1.27524200 \\ \mathrm{C} & 2.73602900 & -1.19355600 & 0.42811200 \\ \mathrm{H} & 1.23341300 & -1.66061600 & -1.04845700 \\ \mathrm{H} & 0.80694200 & -2.17942600 & 0.58372100 \\ \mathrm{C} & 2.59705100 & 1.25490400 & -0.14071900 \\ \mathrm{H} & 1.07962200 & 0.87974400 & -1.63362400 \\ \mathrm{H} & 0.59225300 & 2.02050600 & -0.37874600 \\ \mathrm{C} & 3.39112000 & -0.03833300 & -0.33055600 \\ \mathrm{H} & 3.28755800 & -2.12524700 & 0.25778900 \\ \mathrm{H} & 2.78275000 & -0.98909100 & 1.50724300 \\ \mathrm{H} & 3.05104400 & 2.07076500 & -0.71424200 \\ \mathrm{H} & 2.63703700 & 1.54964200 & 0.91771300 \\ \mathrm{H} & 4.42640000 & 0.09969300 & 0.00118000 \\ \mathrm{H} & 3.42789900 & -0.28493200 & -1.40136100 \\ \mathrm{Ener} & & & \end{array}$

Energy: -537.3326576 a.u

$-0.97808200$

$-1.54972600$

$-0.97455600$

$-1.86951300$

$-2.41886100$

$-0.93434500$

$-4.90898100$

2.45753200

0.46746300

1.27429300

1.13279100

1.23341300

0.80694200

2.59705100

3. 391120

3.28755800

.78275000

3.05104400

3.42789900
$-0.28959200$

.12552100

.68670800

$-0.45856800$

$-1.43164200$

0.15355500

$-1.19355600$
$-0.25346100$

0.57673700

0.96652300

1.63881500

1.36281400

$-0.99757900$

$-0.66443900$

0.267

0.19909900

$-0.55249800$

1.27524200

0.42811200

0.58372100

$-0.14071900$

$-1.63362400$

$-0.37874600$

$-0.33055600$

.25778900

0.91771300

$-1.40136100$

\section{TS-3b}



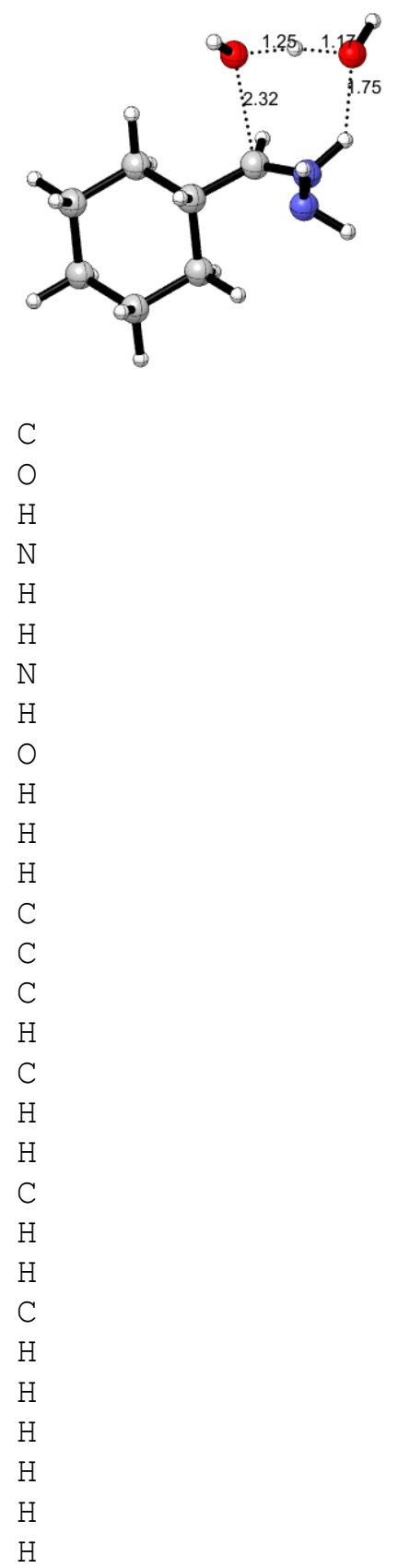

\begin{abstract}
0.01066500
0.31776600

1.08477400

$-2.01127700$

$-2.23439600$

$-2.39908900$

$-0.61082700$

1.30359900

0.51755100

0.44801900

$-0.11783100$

$-0.41544200$

$-0.59680000$

0.24783300

$-0.73597700$

$-1.60353000$

$-0.34529500$

1.26726200

0.32352700

$-1.32392000$

0.25688500

$-1.36647100$

$-0.49194100$

0.28404500

$-1.33298400$

$-1.38878400$

$-2.34979800$

$-0.94949900$

0.50611200
\end{abstract}

$-0.04015800$

$-0.12786600$

0.01931500

2. 16708100

$-0.12441800$

0.00521000

$-1.21081400$

$-0.36067400$

$-2.02180300$

$-0.93349600$

$-1.38420900$

0.56833200

$-1.16999800$

$-0.23191600$

$-2.77214100$

2.13899500

$-2.35010400$

1.77972600

$-1.21887400$

2.08333000

$-1.98412500$

0.19555800

0.25514400

2. 74701500

1.27921200

$-0.45192700$

2.43432300

0.09436400

1.37851100

$-1.98981300$

1. 31424800

$-0.01838300$

3. 78312000

$-0.31374600$

2.34757500

$-0.30976400$

2.33865500

1.18073800

2.73375900

$-2.38776300$

1.25977500

$-2.44596600$

0.56038100

$-2.35313000$

3.89133300

$-1.83298400$

4.59461100

0.06769000

3.90045300

0.15431900

2.79696400

$-3.47958300$

2.80492100

$-2.00059000$

4.84909200

$-2.10481800$

3.87070400

$-2.29363500$

\title{
Energy: -537.2773289 a.u
}

\section{Hydrazone 2b}

$\mathrm{C}$
$\mathrm{O}$
$\mathrm{H}$
$\mathrm{N}$
$\mathrm{H}$
0.59263100

2.96167200

0.71128500

1.62625600

2.54652500
$-0.89991100$

1.98002100

$-1.22673000$

$-0.50316000$

$-0.20007000$
$-0.31191300$

$-0.53307000$

$-1.34626300$

1.70015700

1.99875900 
Energy: -537.3263549 a.u

\section{Reactant complex $2 b$}

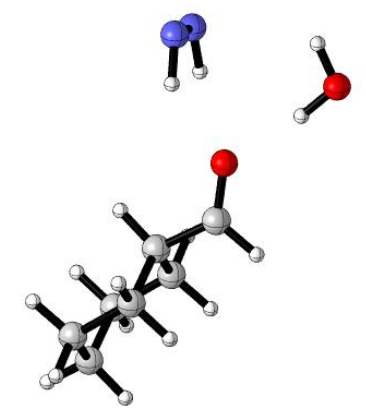

0.93247000

1.67524200

4.58925800

4.28274200

3.55227500

3.40097300

2.07522600

$-0.78072200$

$-0.99590500$

$-1.85793400$

$-0.87399500$

$-2.40232300$

$-0.85445800$

$-0.24067900$

$-3.26418800$

$-1.73534100$

$-1.71258700$

$-3.47341600$

$-2.53795800$

$-2.51070500$

$-4.01225700$

$-3.40982200$

$-4.47159900$

$-3.42326900$
0.20700400

$-0.86476300$

$-1.03120300$

$-0.54927900$

1.20896200

$-0.91168300$

1.59923200

$-0.51455300$

1.00624600

$-1.28339200$

$-0.77105800$

1.41413800

1.27162900

1.56148400

$-0.87373500$

$-1.07194700$

$-2.36165100$

0.63545200

2. 49196100

1.21589500

$-1.41966700$

$-1.16149600$

0.91615400

0.90723400
1.92394200

0.36941000

$-1.32355700$

$-0.54684300$

$-0.59838700$

$-0.30613500$

$-0.51537200$

0.15935000

0.00453700

$-0.61708100$

1.22589400

0.44908100

$-1.05311600$

0.57525200

$-0.17552600$

$-1.68947100$

$-0.48342000$

$-0.31783700$

0.30719600

1. 52463300

$-0.76086200$

0.87519400

0.03613400

$-1.38175300$
C

O

$\mathrm{H}$

$\mathrm{N}$

$\mathrm{H}$

$\mathrm{N}$

$\mathrm{H}$

O

$\mathrm{H}$

$\mathrm{H}$

C

C

C

$\mathrm{H}$
$-0.40966300$

$-1.54775700$

$-0.25667500$

$-3.48174300$

$-2.65424200$

$-3.50999000$

$-2.70022500$

$-4.15964700$

$-3.25566600$

$-4.73464800$

0.82510500

1.57518200

1.71232800

0.52599400
$-0.88674000$

$-0.44522400$

$-1.98053400$

1.63083100

1.10834200

1.65714900

1.15045200

$-1.71094600$

$-1.37009900$

$-0.93743600$

$-0.04978100$

$-0.38777300$

$-0.33535500$

1.00619500
$-0.12883200$

$-0.09049700$

$-0.19840200$

0.63341700

0.98412400

$-0.60152400$

$-1.01096900$

0.07664100

$-0.00598200$

0.03738400

$-0.06774500$

1.23684000

$-1.29407600$

$-0.05822400$ 


$\mathrm{C}$
$\mathrm{H}$
$\mathrm{H}$
$\mathrm{C}$
$\mathrm{H}$
$\mathrm{H}$
$\mathrm{C}$
$\mathrm{H}$
$\mathrm{H}$
$\mathrm{H}$
$\mathrm{H}$
$\mathrm{H}$
$\mathrm{H}$

Energy: -536.0732719 a.u
2.89931900
0.37436600
1.31804300
1.77319200
$-1.46885400$
1.26310900
0.94248600
$-0.15146400$
2.09952000
3.03624600
0.42642200
$-1.19929600$
1. 91623700
1.17691500
3.77717100
3.42447300
2. 69159300
3.65770900
2.83524800
4.70700800
4.05783900
$-1.41460100$
$-0.06522400$
0.09432400
0.09871000
1.45149200
0.18619300
1.50580700
0.66999800
$-0.96825500$
$-1.34156100$
$-2.21093000$
0.09684100
2.23881700
1.37701400
$-2.06855700$
$-1.23699900$
0.15847800
0.09012400

\section{TS-4b}

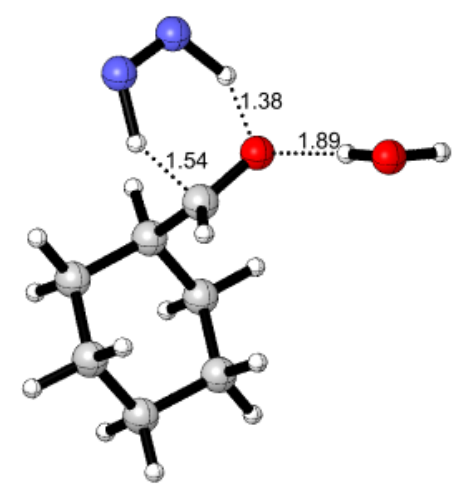

$-0.14706400$

0.17633200

0.61891200

$-1.07215700$

$-0.45196300$

$-1.30173000$

$-0.82238400$

2.96142000

2.01404200

3. 31056200

$-1.31995300$

$-0.94905700$

$-1.77942800$

$-2.13301300$

0.06408300

$-0.57309300$

$-1.87224700$

$-0.77407800$

$-1.98050500$

$-2.73382000$

$-0.44812000$

1.02028300

0.26607300

$-1.18011800$
$-0.33262000$

0.41524600

$-1.02220200$

$-0.91532800$

$-0.07481000$

$-1.75285600$

$-1.49461200$

0.22825400

0.36049000

1.09580900

0.04953300

1.29088600

$-1.10191800$

0.33355400

0.96252000

2.08003500

1.66537900

$-1.41520500$

$-2.00018500$

$-0.80866200$

$-0.16489600$

0.66192500

1.86199900

$-2.20065800$
0.06059600

1.04081500

$-0.32564800$

2. 65056000

2.16376200

1. 84823400

0.81330800

1.64031400

1.47226200

1.87296400

$-0.81790800$

$-1.65976900$

$-1.72327200$

$-0.13577900$

$-2.75952300$

$-0.99986900$

$-2.12115300$

$-2.83822900$

$-1.12563100$

$-2.17880100$

$-3.65838000$

$-2.30769600$

$-3.35132700$

$-3.48498000$ 


\section{$\mathrm{H}$ \\ $\mathrm{H}$}

$\mathrm{H}$

Energy: -536.0531784 a.u
0.15452700
$-1.81532400$
$-0.40039200$
0.17417600
$-2.40909700$
$-4.43217200$
$-4.17565300$

\section{Prod 4b}

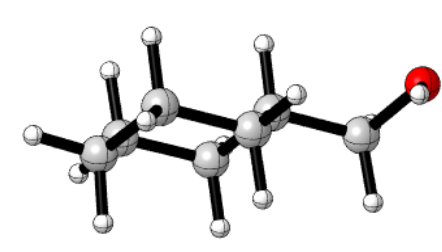

C

O

$\mathrm{H}$

N

$\mathrm{H}$

N

$\mathrm{H}$

O

$\mathrm{H}$

$\mathrm{H}$

C

C

C

$\mathrm{H}$

C

$\mathrm{H}$

$\mathrm{H}$

C

$\mathrm{H}$

$\mathrm{H}$

C

$\mathrm{H}$

$\mathrm{H}$

$\mathrm{H}$

$\mathrm{H}$

$\mathrm{H}$

$\mathrm{H}$

$$
\begin{array}{r}
1.18987900 \\
2.13403700 \\
1.55109800 \\
0.71417300 \\
1.89960100 \\
0.08319800 \\
1.20297000 \\
4.74786700 \\
3.86427900 \\
5.16015000 \\
-0.22322100 \\
-1.15110300 \\
-0.78498700 \\
-0.18100000 \\
-2.57885600 \\
-1.15988100 \\
-0.75934800 \\
-2.20705700 \\
-0.79271900 \\
-0.14192100 \\
-3.13330800 \\
-3.22796700 \\
-2.57497200 \\
-2.59230700 \\
-2.18397500 \\
-4.13783300 \\
-3.23109400
\end{array}
$$

$-1.04392600$

0.00145400

$-1.55145200$

2. 96041100

0.45057800

2.97217400

$-1.76959800$

$-1.03115500$

$-0.62737600$

$-0.62944000$

$-0.52287200$

$-1.65944500$

0.15371600

0.21983600

$-1.15729800$

$-2.43722000$

$-2.12475400$

0.67034400

$-0.58259800$

0.98206000

$-0.45866200$

$-1.99080200$

$-0.44729900$

1.13338300

1.45487000

$-0.06890700$

$-1.19290000$
0.01736300

0.26875200

$-0.88260200$

$-0.10326200$

1.09208500

$-1.00211800$

0.84386600

0.09194200

0.16020500

$-0.68064900$

$-0.20174700$

$-0.64465600$

1.05429600

$-1.01372900$

$-0.87633800$

0.13385900

$-1.55741900$

0.82431900

1.87279100

1.37917200

0.36774400

$-1.16714800$

$-1.71537300$

1.73959800

0.05363800

0.16798300

1.17994000

Energy: -536.1710399 a.u 


\section{References}

27. Zhao, Y.; Truhlar, D. G., The M06 suite of density functionals for main group thermochemistry, thermochemical kinetics, noncovalent interactions, excited states, and transition elements: two new functionals and systematic testing of four M06-class functionals and 12 other functionals. Theor. Chem. Account 2008, 120 (1), 215-241.

28. Marenich, A. V.; Cramer, C. J.; Truhlar, D. G., Universal Solvation Model Based on Solute Electron Density and on a Continuum Model of the Solvent Defined by the Bulk Dielectric Constant and Atomic Surface Tensions. J. Phys. Chem. B 2009, $113(18), 6378-6396$

29. Fukui, K., The path of chemical reactions - the IRC approach. Acc Chem Res 1981, 14, 363.

30. Legault, C. Y. CYLview, 1.0b; Universitéde Sherbrooke: Canada, 2009; http://www.cylview.org

31. Frisch, M. J., Trucks, G. W., Schlegel, H. B., Scuseria, G. E., Robb, M. A., Cheeseman, J. R., Scalmani, G., Barone, V., Petersson, G. A., Nakatsuji, H., Li, X., Caricato, M., Marenich, A. V., Bloino, J., Janesko, B. G., Gomperts, R., Mennucci, B., Hratchian, H. P., Ortiz, J. V., Izmaylov, A. F., Sonnenberg, J. L., Williams-Young, D., Ding, F., Lipparini, F., Egidi, F., Goings, J., Peng, B.; Petrone, A., Henderson, T., Ranasinghe, D., Zakrzewski, V. G., Gao, J., Rega, N., Zheng, G., Liang, W., Hada, M., Ehara, M., Toyota, K., Fukuda, R., Hasegawa, J., Ishida, M., Nakajima, T., Honda, Y., Kitao, O., Nakai, H., Vreven, T., Throssell, K., Montgomery, J. A., Jr., Peralta, J. E., Ogliaro, F., Bearpark, M., Heyd, J. J., Brothers, E., Kudin, K. N., Staroverov, V. N., Keith, T. A., Kobayashi, R., Normand, J., Raghavachari, K., Rendell, A. P., Burant, J. C., Iyengar, S. S., Tomasi, J., Cossi, M., Millam, J. M., Klene, M., Adamo, C., Cammi, R., Ochterski, J. W., Martin, R. L., Morokuma, K., Farkas, Ö., Foresman, J. B., Fox, D. J. Gaussian 16, Revision A.03, Gaussian, Inc., Wallingford CT, 2016. 


\section{GC and GC-MS}

a) Thenmozhia, S., Kadirvelu, K. Transfer Hydrogenation and Hydration of Aromatic Aldehydes and Nitriles Using Heterogeneous NiO Nanofibers as a Catalyst. New J. Chem., 2018, 42, 15572-15577; b) Zhang, G., Scott, B., Hanson, S. Mild and Homogeneous Cobalt-Catalyzed Hydrogenation of $\mathrm{C}=\mathrm{C}, \mathrm{C}=\mathrm{O}$, and $\mathrm{C}=\mathrm{N}$ Bonds. Angew, Chem. Int. Ed. 2012, 51, 12102-12106; c) Salvano, M., Cantero, J., Vázquez, A., Formica, S., Aimard, M. Searching for local biocatalysts: Bioreduction of aldehydes using plant roots of the Province of Córdoba (Argentina). J. Mol. Catal. B: Enzym. 2011, 71, 16-21; d) John M. Ovian†, Kelly, C., Pistritto, V., Leadbeater, N. Accessing N-Acyl Azoles via Oxoammonium Salt-Mediated Oxidative Amidation. Org. Lett. 2017, 19, 1286-1289; e) Lin,Y., Zhu, D-P., Du, Y-R., Zhang, R., Zhang, S-J., Xu, B-H. Tris(pyrazolyl)borate Cobalt-Catalyzed Hydrogenation of $\mathrm{C}=\mathrm{O}, \mathrm{C}=\mathrm{C}$ and $\mathrm{C}=\mathrm{N}$ Bonds: An Assistant Role of Lewis Base. Org. Lett. 2019, 21, 2693-2698; f) Xu,W., Fan,H., Wu, G., Chen, P. Comparative study on reducing aromatic aldehydes by using ammonia borane and lithium amidoborane as reducing reagents. New J. Chem., 2012, 36, 1496-1501; g) Everson, D., Shrestha, R., Weix, D. Nickel-Catalyzed Reductive Cross-Coupling of Aryl Halides with Alkyl Halides. J. Am. Chem. Soc. 2010, 132, 920-921; h) Sohail Ahmad, M., He, H., Nishina, Y. Selective Hydrogenation by Carbocatalyst: The Role of Radicals. Org. Lett. 2019, 21, 8164-8168; i) Choudhary, N., Ghosh, T., Mobin, S. Ketone Hydrogenation by Using $\mathrm{ZnO} \mathrm{Cu}(\mathrm{OH}) \mathrm{Cl} / \mathrm{MCM}-41$ with a Splash of Water: An Environmentally Benign Approach. Chem. - Asian J. 2020, 15(8), 1339-1348; j) Böldl, M., Fleischer, I. Dehydrative Coupling of Benzylic Alcohols Catalyzed by Brønsted Acid/Lewis Base. Eur. J. Org. Chem., 2019, 34, 5856-5861; k) Koul, s., Koul, J., Singh, B., Kapoor, M., Parshad, R., Manhas, K., Taneja, S., Qazi., G. Trichosporon beigelli esterase (TBE): a versatile esterase for the resolution of economically important racemates. Tetrahedron: Asymmetry, 2005, 16, 2575-2591; 1) Moore, P., Mirzayans, P., Williams, C. NMO-TPB: A Selectivity Variation on the Ley-Griffith TPAP Oxidation. Chem. Eur. J. 2014, $21,3567-3571$. 


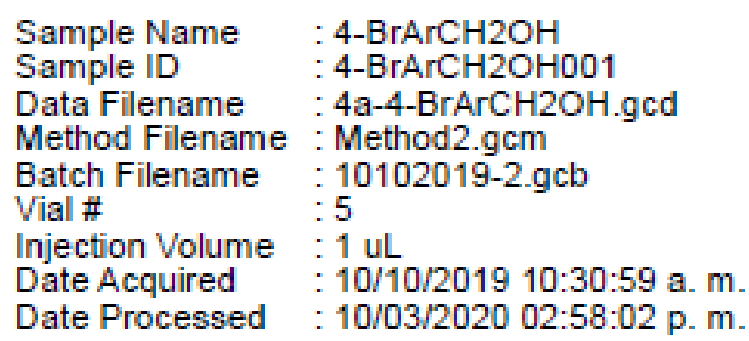

uV

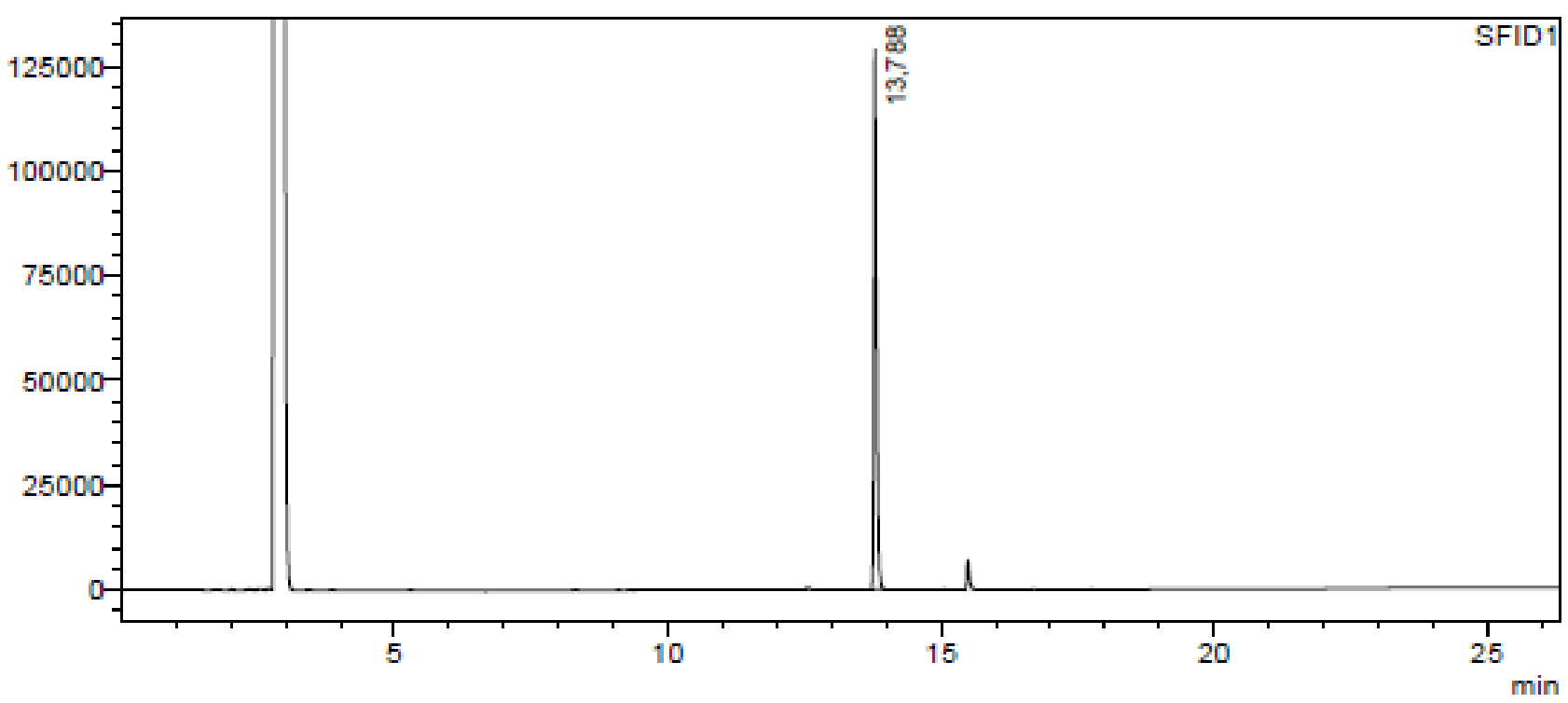

SFID1

\begin{tabular}{|r|r|r|r|r|r|r|r|}
\hline Peak\# & Ret. Time & Area & Height & Conc. & Unit & Mark & Name \\
\hline 1 & 13,788 & 444051 & 128492 & 100,000 & & $\mathrm{M}$ & \\
\hline Total & & 444051 & 128492 & & & & \\
\hline
\end{tabular}


$\begin{array}{ll}\text { Sample Name } & \text { : benzylalcohol } \\ \text { Sample ID } & \text { : benzylalcohol } \\ \text { Data Filename } & \text { : benzylalcohol.gcd } \\ \text { Method Filename } & : \text { Method } 2 . g c m \\ \text { Batch Filename } & : 13112019 . \mathrm{gcb} \\ \text { Vial \# } & : 2 \\ \text { Injection Volume } & : 1 \mathrm{uL} \\ \text { Date Acquired } & : 13 / 11 / 2019 \text { 07:23:24 a. m. } \\ \text { Date Processed } & : 13 / 11 / 2019 \text { 07:49:47 a. m. }\end{array}$
Sample Type : Unknown

Acquired by : System Administrator Processed by : System Administrator

uV

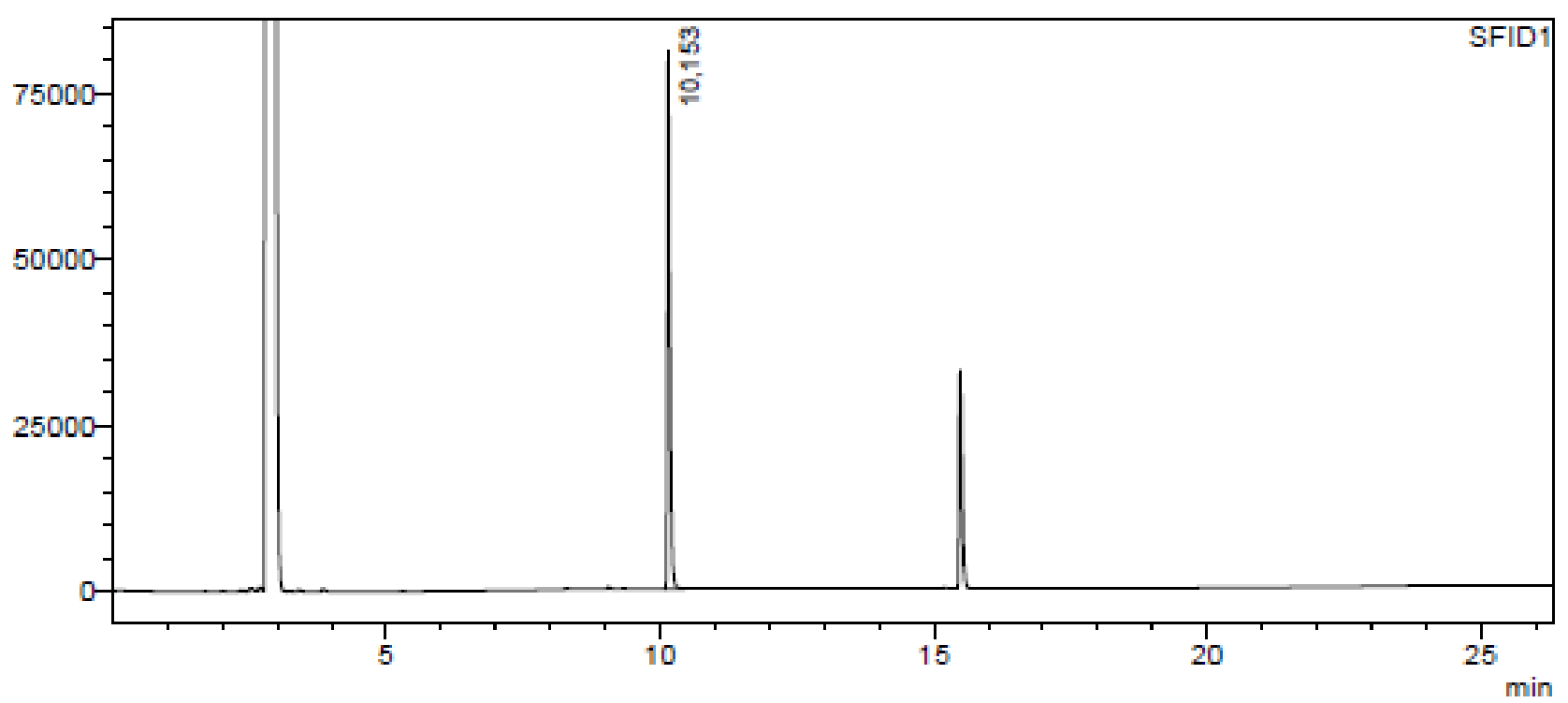

\begin{tabular}{|c|c|c|c|c|c|c|c|}
\hline Peak\# & Ret. Time & Area & Height & Conc. & Unit & Mark & Name \\
\hline 1 & 10,153 & 275492 & 81069 & 100,000 & & $\mathrm{~S}$ & \\
\hline Tota & & 275492 & 81069 & & & & \\
\hline
\end{tabular}


Sample Name

Sample ID

Data Filename

Method Filename

Batch Filename

Vial \#

Injection Volume

Date Acquired

Date Processed : $09 / 03 / 2020$
SLS-176

SLS-176

4c-SLS-176.gcd

: Method2-1min-eq.gcm

:05032020.gcb

$: 1$

$: 1$ uL

: 05/03/2020 02:40:48 p. m. : 09/03/2020 04:36:57 p. m.
Sample Type : Unknown

Acquired by : System Administrator Processed by : System Administrator

uV

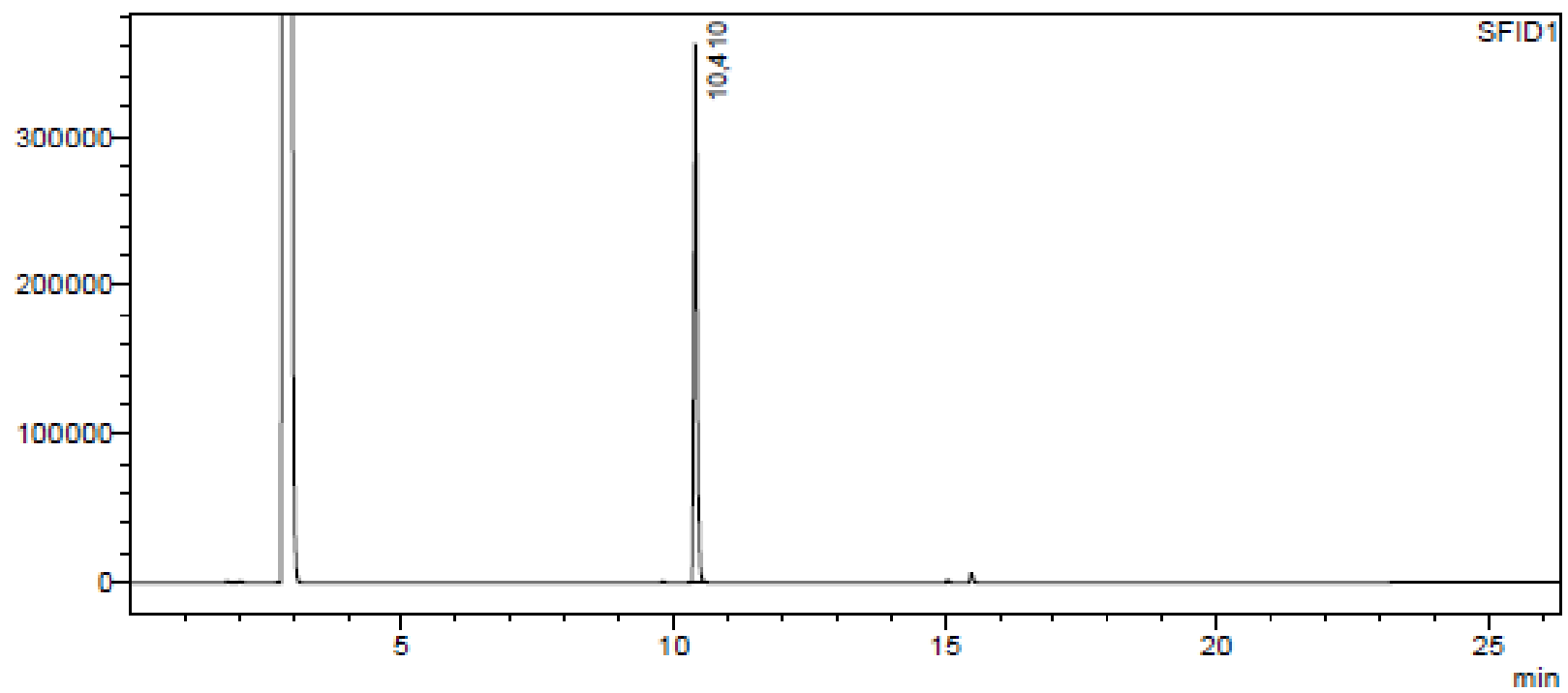

SFID1

\begin{tabular}{|r|r|r|r|r|r|r|r|}
\hline Peak\# & Ret. Time & Area & Height & Conc. & Unit & Mark & Name \\
\hline 1 & 10,410 & 1418391 & 360404 & 100,000 & & $\mathrm{M}$ & \\
\hline Tota & & 1418391 & 360404 & & & & \\
\hline
\end{tabular}


Analysis Date \& Time : $25 / 11 / 2016$ 10:42:26 a.m.

User Name

Vial\#

Admin

Sample Name

Sample ID

$: 2$

NC-Red-11

MA- 55

Sample Type

Injection Volume

: Unknown

ISTD Amount

1

Data Name

Method Name

C:IUsers IShimadzu GCDDocuments Productos-JOC-4d-NC-Red-11.gcd

C:IGCsolution'Data Project1ISUSANLétodosLMethod2.gcm

Intensity

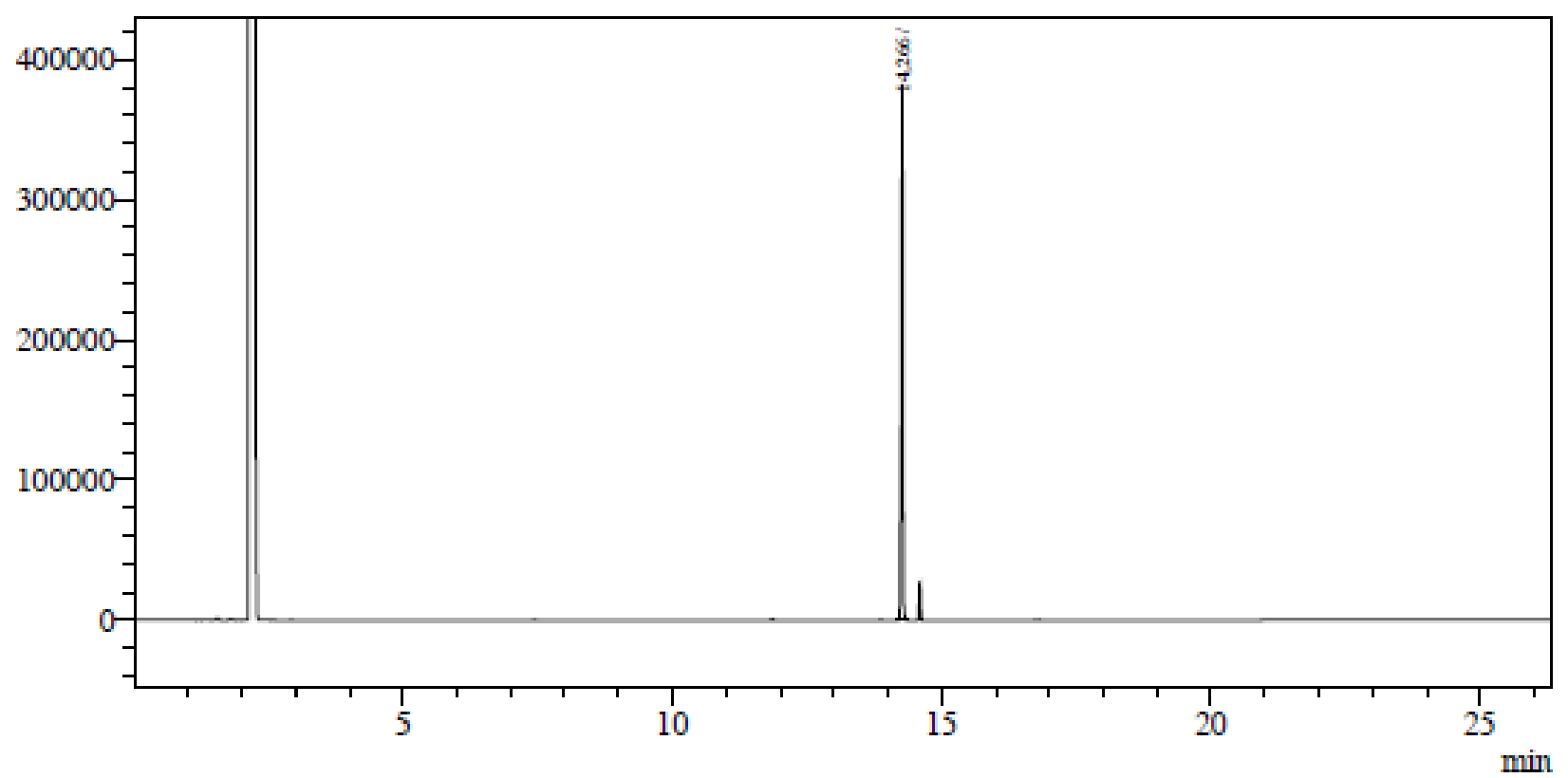

FID1

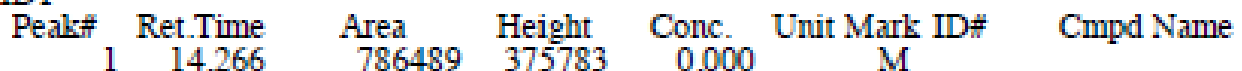

Total $\quad 786489 \quad 375783$ 
Analysis Date \& Time $\quad: 29 / 12 / 2016$ 11:00:36 a.m.

User Name

Vial\#

Sample Name

Sample ID

Admin

Sample Type

$: 6$

Injection Volume

ISTD Amount

: MA-56

MA-56

:Unknown

$: 1$

1

\section{Data Name}

C:IUsers'Shimadzu GCDDocuments'Productos-JOC/4e-MA-56.gcd

Method Name

: C:IGCsolution'Data Projectl ISUSANM Métodos/Method2.gcm

Intensity

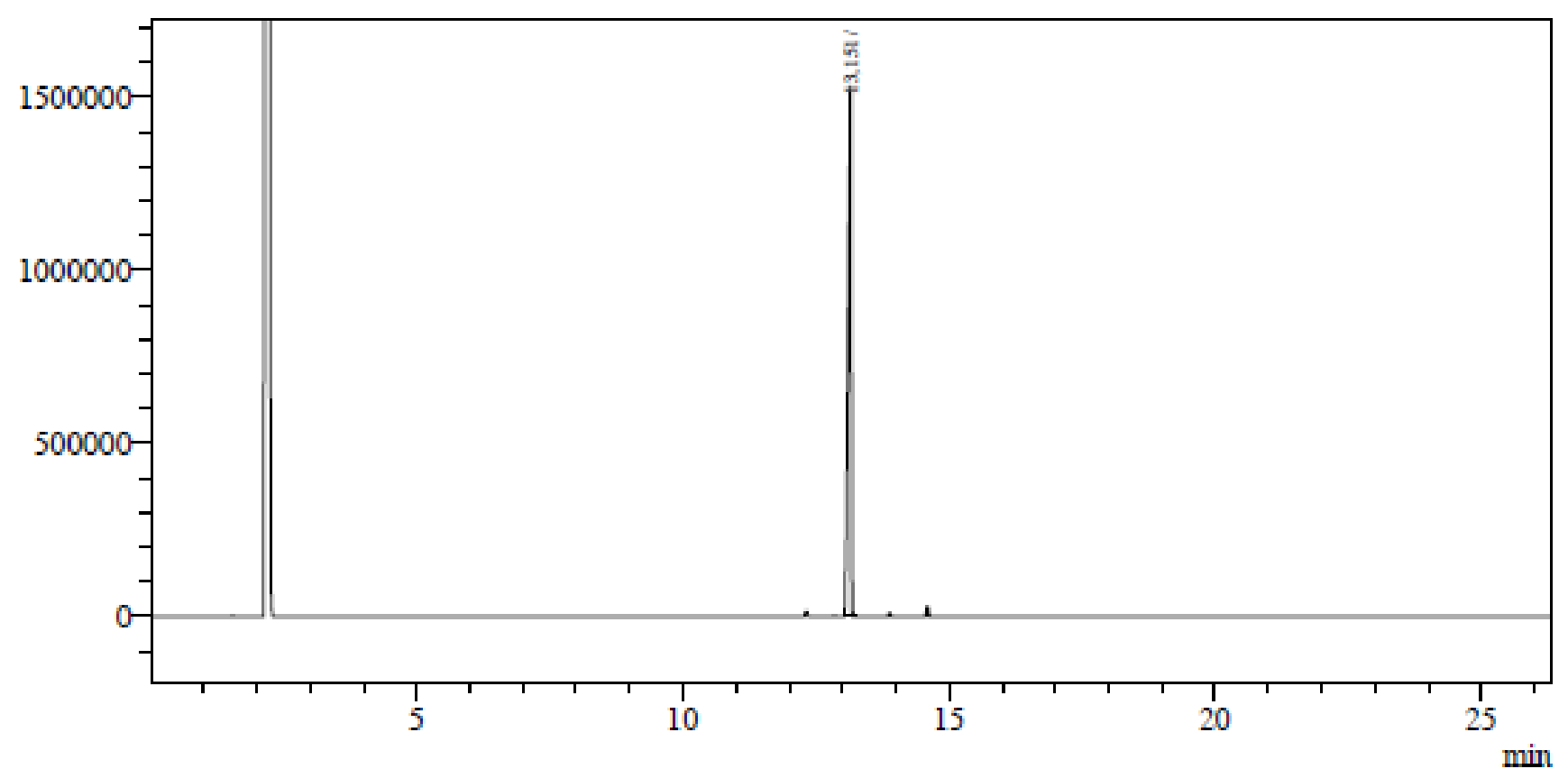

FIDl

Peals\# Ret.Time Area Height Conc. Unit Mark ID\# Cmpd Name

$\begin{array}{lrrr}1 & 13,151 & 5681972 & 1505461\end{array}$

$5681972 \quad 1505461$ 
Sample Name

Sample ID

Data Filename

Method Filename

Batch Filename

Vial \#

Injection Volume : : $1 \mathrm{uL}$

Date Acquired : 05/03/2020 01:57:12 p. m.

Date Processed $\quad: 09 / 03 / 2020$ 04:36:12 p. m.
: SLS-175

SLS-175

4f-SLS-175.gcd

Method2-1 min-eq.gcm

$05032020 . \mathrm{gcb}$
Sample Type : Unknown

Acquired by : System Administrator

Processed by : System Administrator

uV

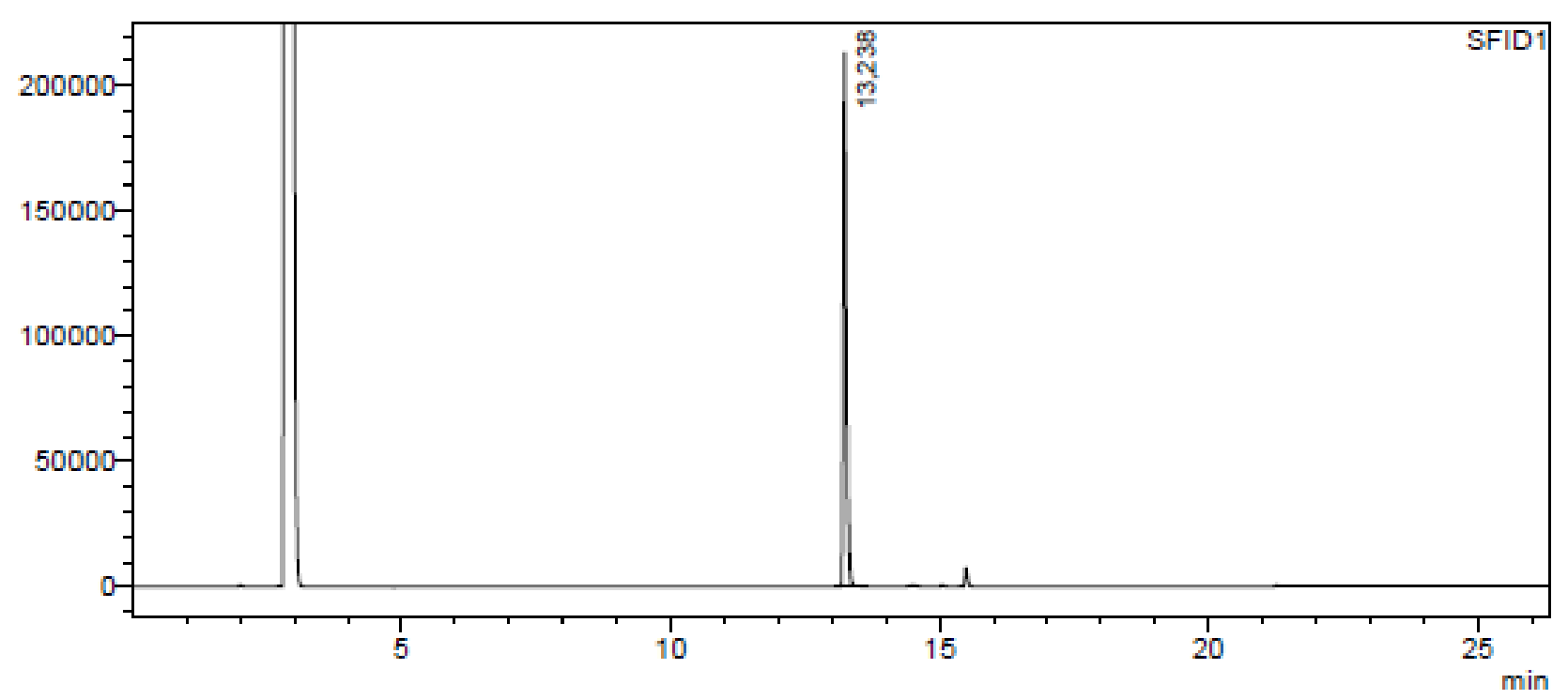

\section{SFID1}

\begin{tabular}{|r|r|r|r|r|r|r|}
\hline Peak\# & Ret. Time & \multicolumn{1}{|c|}{ Area } & \multicolumn{1}{c|}{ Height } & \multicolumn{1}{c|}{ Area\% } & \multicolumn{1}{c|}{ Conc. } & Name \\
\hline 1 & 13,238 & 783626 & 212359 & 100,000 & 100,000 & \\
\hline Total & & 783626 & 212359 & 100,000 & & \\
\hline
\end{tabular}


Sample Name : SLS-171

Sample ID : :SLS-171

Data Filename : 4g-SLS-171.gcd

Method Filename : Method2-1min-eq.gcm

Batch Filename : $05032020 . \mathrm{gcb}$

Vial \# : : 2

Injection Volume : $1 \mathrm{uL}$

Date Acquired : :05/03/2020 11:14:54 a. m.

Sample Type : Unknown

Date Processed : 09/03/2020 04:11:49 p. m.

Acquired by : System Administrator

Processed by : : System Administrator

uV

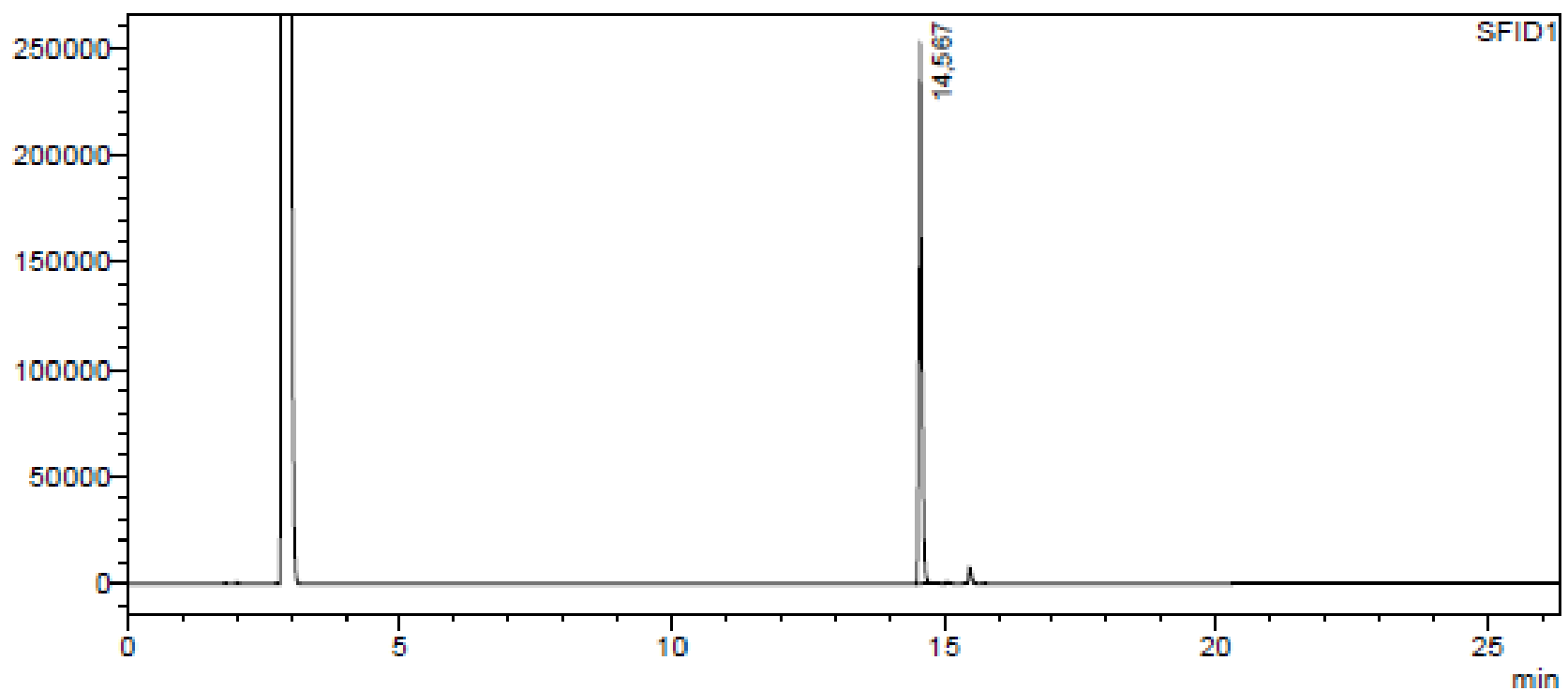

SFID1

\begin{tabular}{|r|r|r|r|r|r|r|}
\hline Peak\# & Ret. Time & \multicolumn{1}{|c|}{ Area } & Height & Area $\%$ & Conc. & Name \\
\hline 1 & 14,567 & 946042 & 250794 & 100,000 & 100,000 & \\
\hline Tota & & 946042 & 250794 & 100,000 & & \\
\hline
\end{tabular}


Sample Name : SLS-190-4hf2

Sample ID

Data Filename : 4h-SLS-190-4hf2.gcd

Method Filename : Method2-1min-eq.gcm

Batch Filename : $12032020 . \mathrm{gcb}$

Vial \# : : 5

Injection Volume : 1 uL

Date Acquired $\quad: 12 / 03 / 2020$ 01:27:34 p. m.

Date Processed : $: 12 / 03 / 2020$ 01:53:56 p. m.
Sample Type : Unknown

Acquired by : : System Administrator

Processed by : : System Administrator

uV

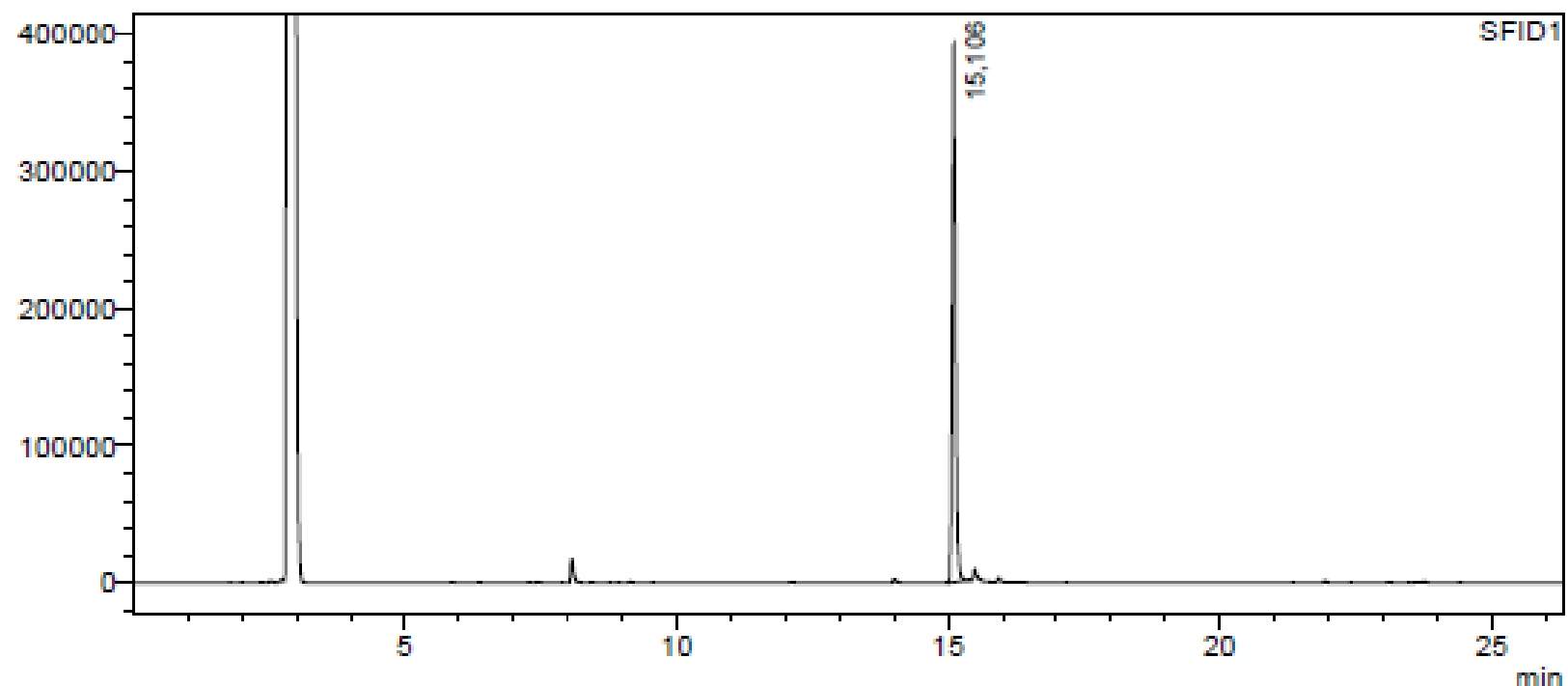

SFID1

\begin{tabular}{|r|r|r|r|r|r|r|}
\hline Peak\# & Ret. Time & \multicolumn{1}{|c|}{ Area } & Height & \multicolumn{1}{c|}{ Area\% } & Conc. & Name \\
\hline 1 & 15,106 & 1629309 & 389916 & 100,000 & 100,000 & \\
\hline Total & & 1629309 & 389916 & 100,000 & & \\
\hline
\end{tabular}


Analysis Date \& Time

User Name

Vial\#

Sample Name

Sample ID

Sample Type

Injection Volume

ISTD Amount

Data Name

Method Name

Intensity

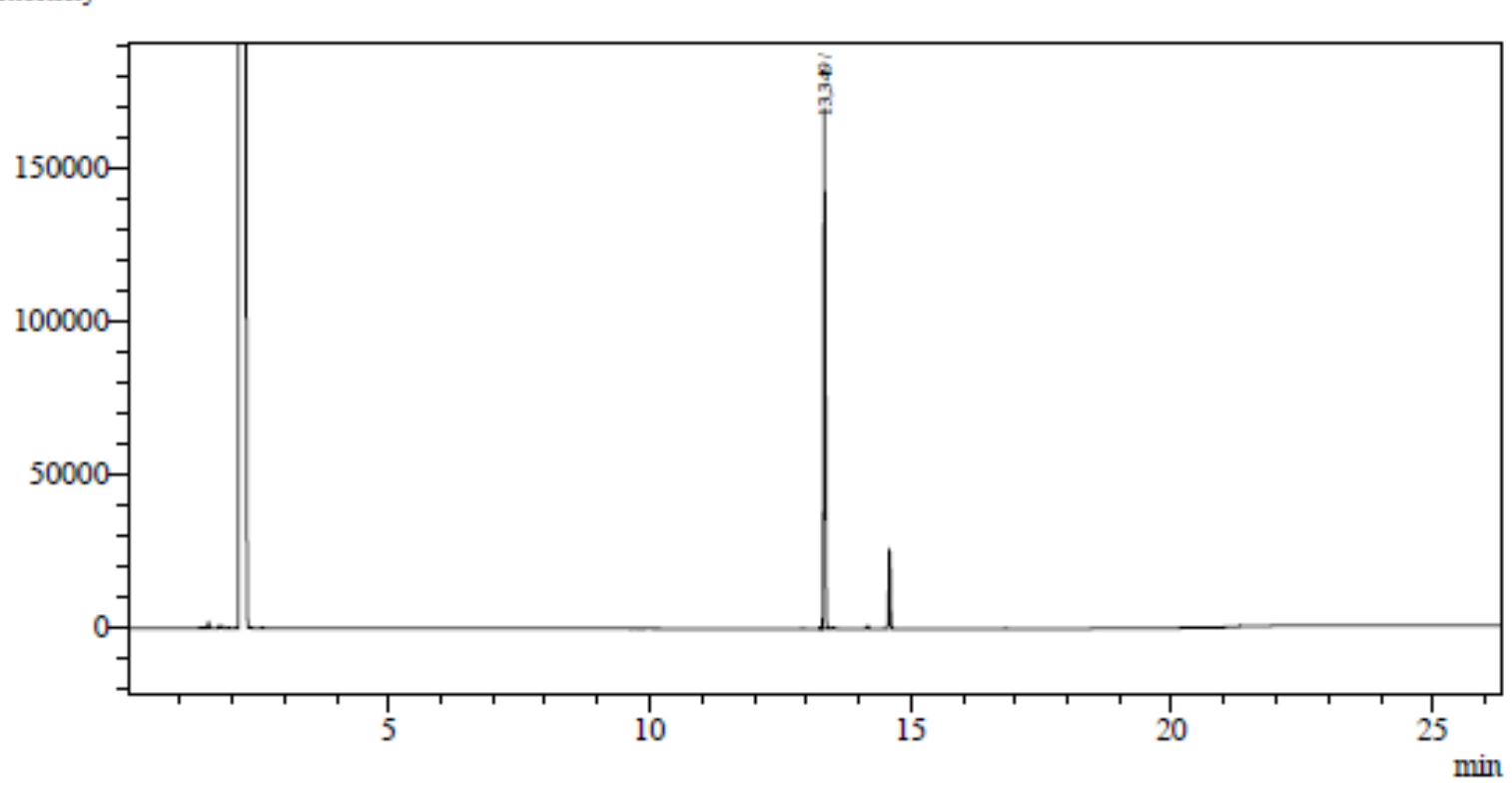

$0 / 11 / 2016$ 11:26:28 a.m.

Admin

2

MA-53-0002

MA-53-0002

Unknown

$: 1$

: C:IUsers Shimadzu GCDDocuments Productos-JOC-4i-MA-52-OH.gcd

: C:IGCsolution Data ProjectllSUSANLMétodos LMethod2.gcm

FID1

\begin{tabular}{rrrrrrr} 
Pealk\# & Ret.Time & \multicolumn{1}{c}{ Area } & Height & Conc. & Unit Mark ID\# & Cmpd Name \\
1 & 13,349 & 314837 & 168859 & 0,000 & $\mathrm{M}$ & \\
\hline Total & & 314837 & 168859 & &
\end{tabular}


Sample Name

Sample ID

Data Filename

Method Filename

Batch Filename

Vial \#

Injection Volume

Date Acquired

Date Processed
: SLS-174

SLS-174

: 4j-SLS-174.gcd

: Method2-1min-eq.gcm

: 05032020.gcb

$: 5$

: 1 uL

05/03/2020 01:14:57 p. m.

: 09/03/2020 04:22:33 p. m.
Sample Type : Unknown

Acquired by : : System Administrator

Processed by : : System Administrator

uV

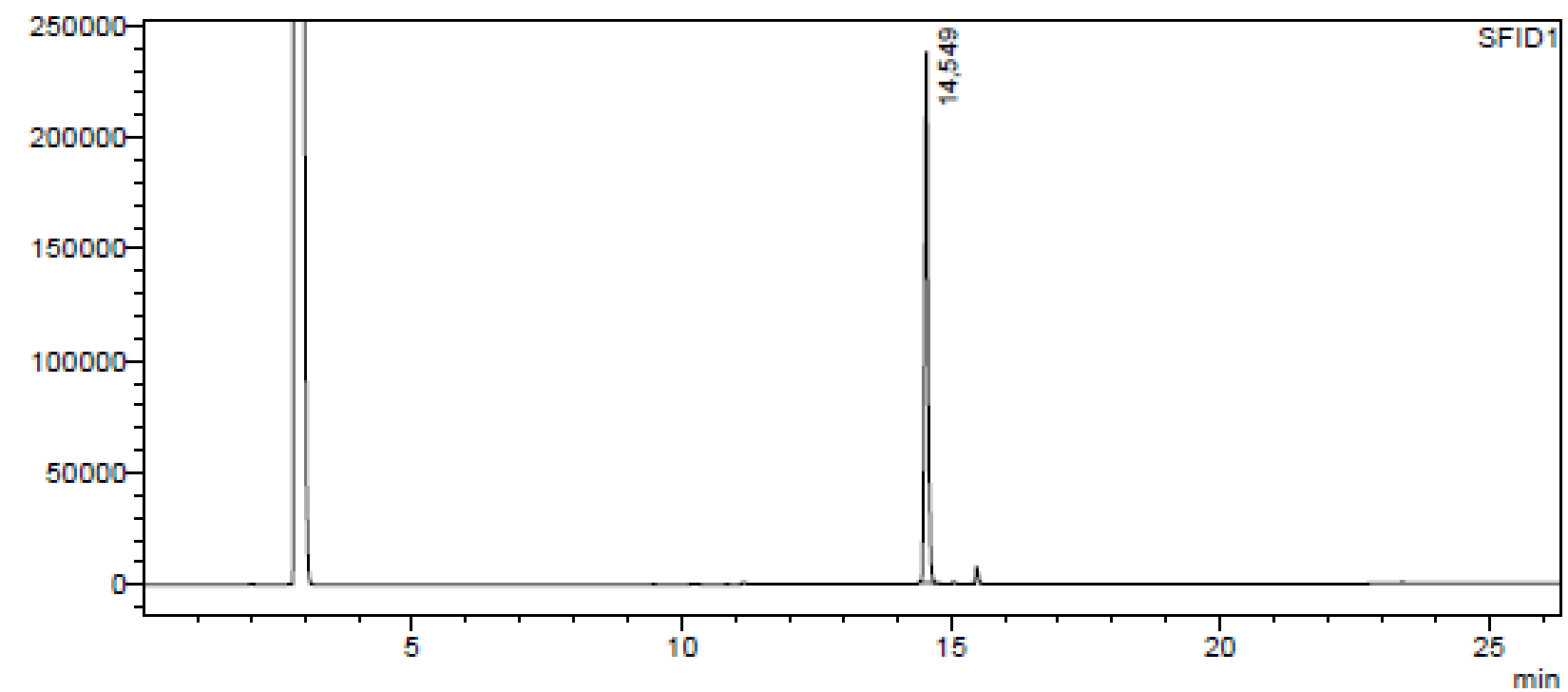

SFID1

\begin{tabular}{|r|r|r|r|r|r|r|}
\hline Peak\# & Ret. Time & Area & Height & Area\% & Conc. & Name \\
\hline 1 & 14,549 & 1009095 & 236916 & 100,000 & 100,000 & \\
\hline Total & & 1009095 & 236916 & 100,000 & & \\
\hline
\end{tabular}


Sample Name : SLS-170-1

Sample ID : :SLS-170-1

Data Filename : 4k-SLS-170-1.gcd

Method Filename : Method2-1min-eq.gcm

Batch Filename $\quad \vdots$
Vial \#

Injection Volume $: 1 \mathrm{uL}$

Date Acquired : :05/03/2020 10:37:15 a. m.

Date Processed : $10 / 03 / 2020$ 02:45:19 p. m.

Sample Type : Unknown

Acquired by : System Administrator Processed by : System Administrator

uV

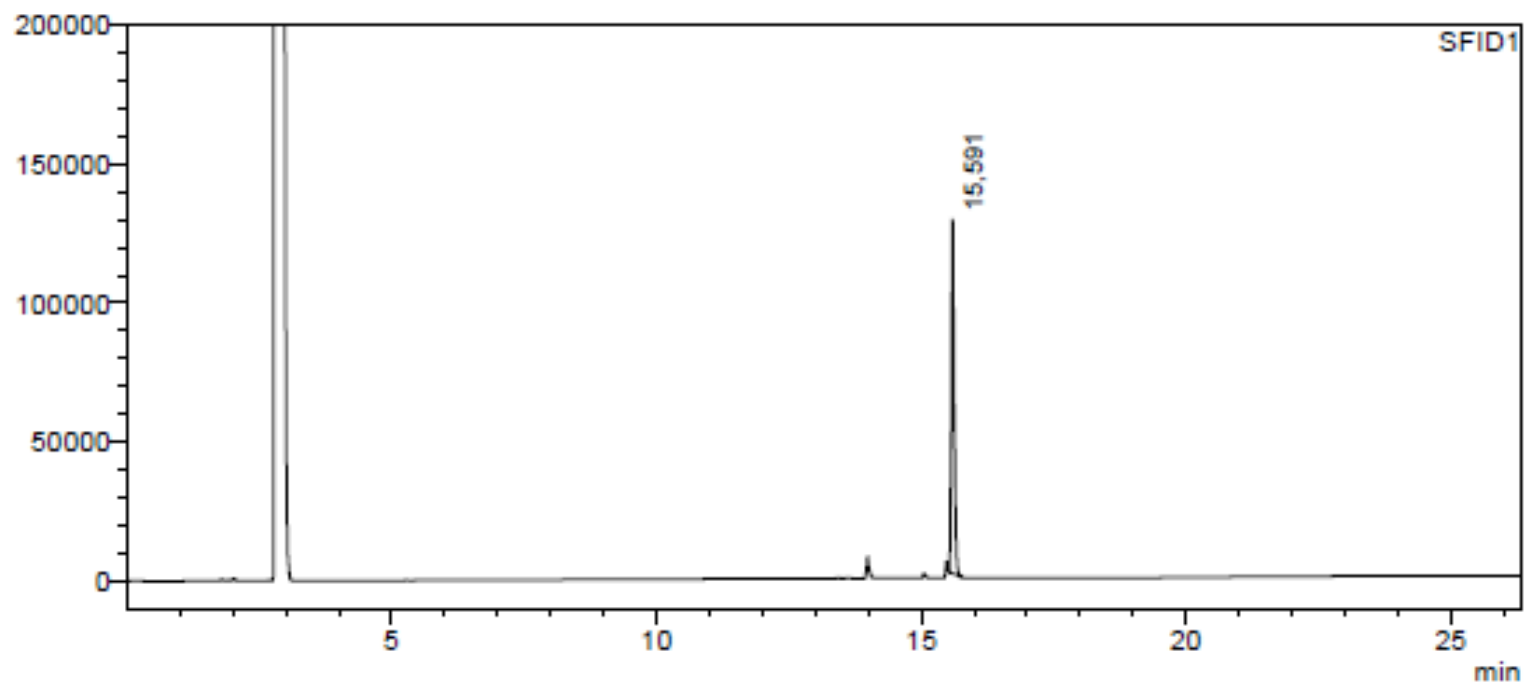

SFID1

\begin{tabular}{|r|r|r|r|r|r|r|}
\hline Peak\# & Ret. Time & Area & Height & Area\% & Conc. & Name \\
\hline 1 & 15,591 & 454929 & 127275 & 100,000 & 100,000 & \\
\hline Total & & 454929 & 127275 & 100,000 & & \\
\hline
\end{tabular}


Sample Name

Sample ID

Data Filename

Method Filename : Method2-1min-eq.gcm

Batch Filename : $06032020 . \mathrm{gcb}$

Vial \# : :2

Injection Volume : $1 \mathrm{uL}$

Date Acquired : 06/03/2020 11:24:37 a. m.

Date Processed $\quad$ : 09/03/2020 04:46:12 p. m.
Sample Type : Unknown

Acquired by : System Administrator Processed by : System Administrator

uV

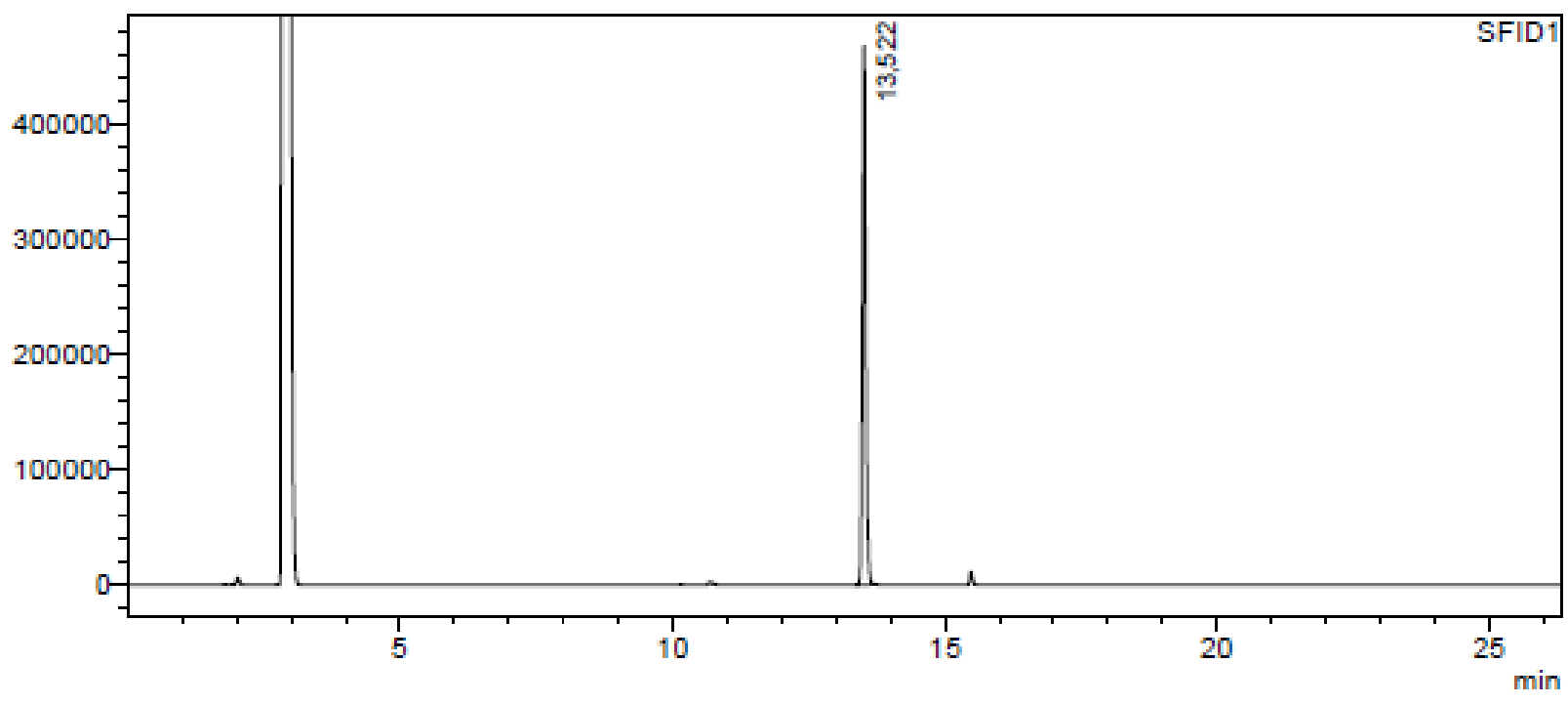

SFID1

\begin{tabular}{|r|r|r|r|r|r|r|}
\hline Peak\# & Ret. Time & \multicolumn{1}{|c|}{ Area } & \multicolumn{1}{c|}{ Height } & \multicolumn{1}{c|}{ Area\% } & \multicolumn{1}{c|}{ Conc. } & Name \\
\hline 1 & 13,522 & 1928847 & 466024 & 100,000 & 100,000 & \\
\hline Total & & 1928847 & 466024 & 100,000 & & \\
\hline
\end{tabular}


Sample Name

Sample ID

Data Filename

: Method 2-1min-eq.gcm

Batch Filename : 05032020.gcb

Vial \#

Injection Volume : 1 uL

Date Acquired : 05/03/2020 03:25:19 p. m.

Date Processed : 09/03/2020 04:41:10 p. m.

$\begin{array}{ll}\text { Sample Type } & \text { : Unknown } \\ \text { Acquired by } & \text { : System Administrator } \\ \text { Processed by } & \text { : System Administrator }\end{array}$

uV

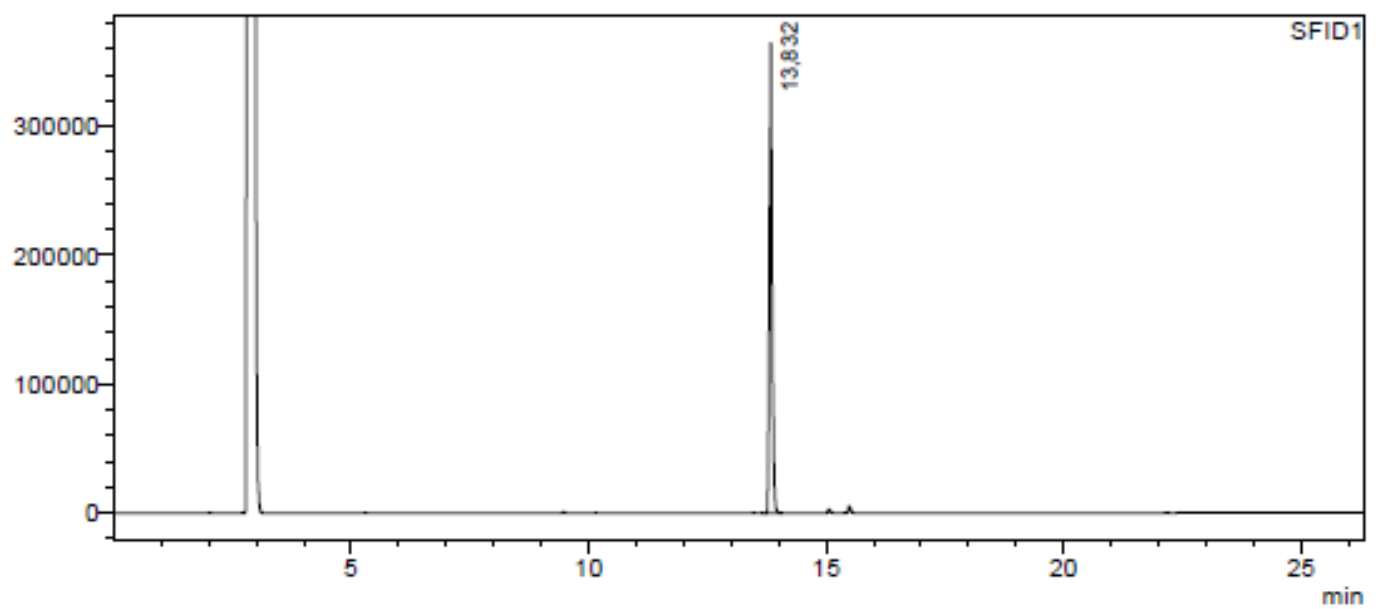

SFID1

\begin{tabular}{|r|r|r|r|r|r|r|}
\hline Peak\# & Ret. Time & \multicolumn{1}{|c|}{ Area } & \multicolumn{1}{c|}{ Height } & \multicolumn{1}{c|}{ Area\% } & \multicolumn{1}{c|}{ Conc. } & Name \\
\hline 1 & 13,832 & 1452619 & 363089 & 100,000 & 100,000 & \\
\hline Tota & & 1452619 & 363089 & 100,000 & & \\
\hline
\end{tabular}


Sample Name

Sample ID

Data Filename

Method Filename

Batch Filename

Vial \#

Injection Volum

Date Acquired

Date Processed

$: 1 \mathrm{uL}$

11/03/2020 10:20:38 a. m.

: 11/03/2020 10:50:18 a. m.
SLS-197

SLS-197

$4 n-S L S-197 . g c d$

Method 2-1min-eq.gcm
Sample Type : Unknown

Acquired by : System Administrator

Processed by : : System Administrator

uV

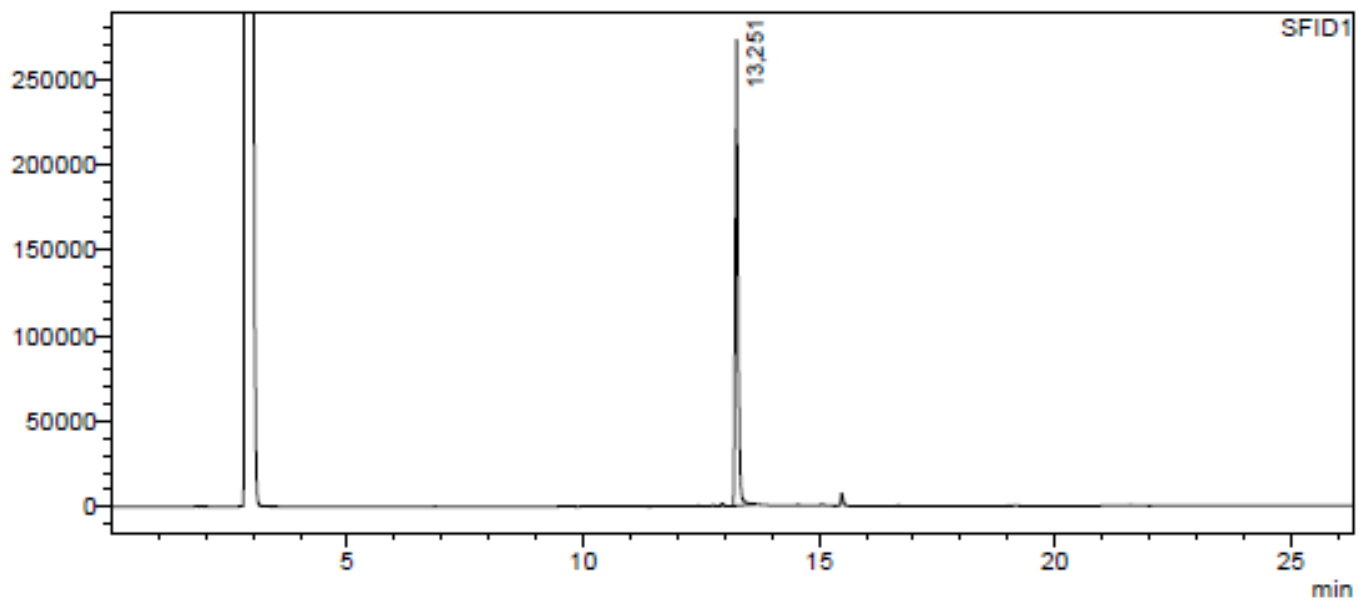

SFID1

\begin{tabular}{|l|l|l|l|l}
\hline Peak\# Ret. Time & Area & Height & Area\% & Conc.
\end{tabular}

\begin{tabular}{r|r|r|r|r|r|}
\hline 1 & 13,251 & 1068992 & 271369 & 100,000 & 100,000 \\
\hline Total & & 1068992 & 271369 & 100,000 &
\end{tabular}

Name 


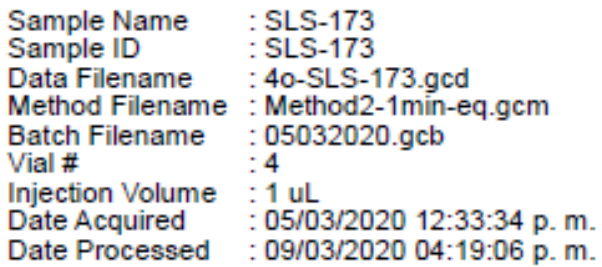

uV

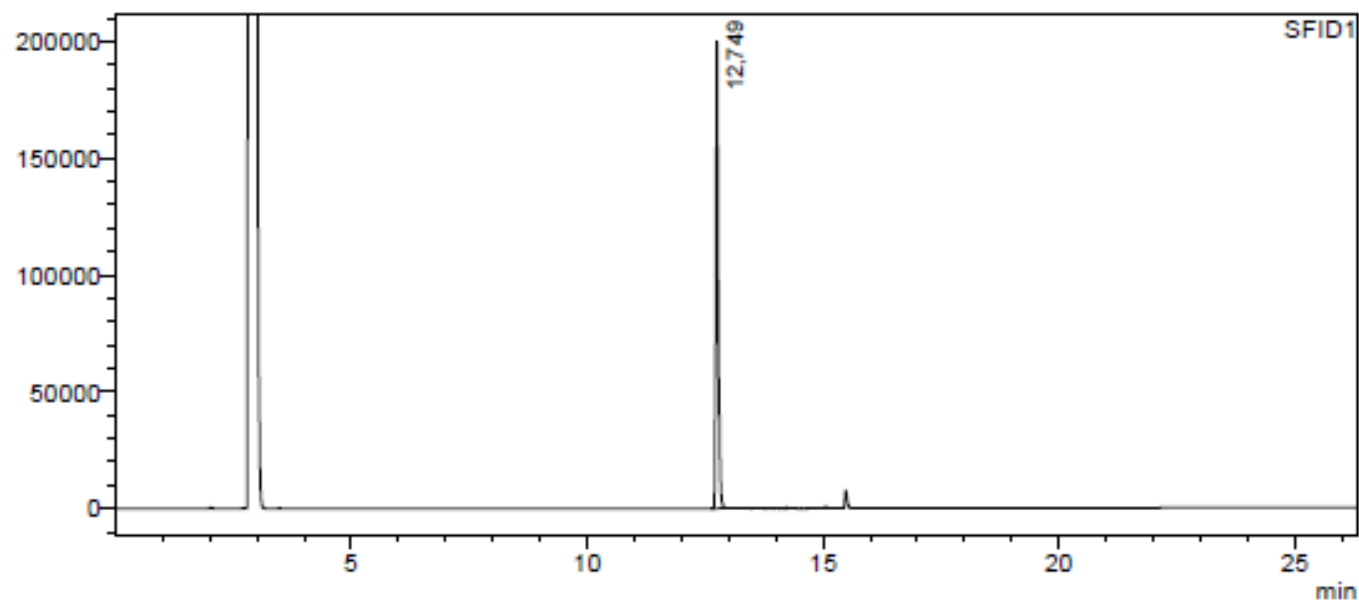

\begin{tabular}{|c|c|c|c|c|c|c|}
\hline Peak\# & Ret. Time & Area & Height & Area $\%$ & Conc. & Name \\
\hline 1 & 12,749 & 704880 & 199313 & 100,000 & 100,000 & \\
\hline Total & & 704880 & 199313 & 100,000 & & \\
\hline
\end{tabular}


Sample Name : SLS-178

Sample ID : SLS-178

Data Filename :4p-SLS-178.gcd

Method Filename : Method2-1min-eq.gcm

Batch Filename : 05032020.gcb

Vial \#

Injection Volume : $1 \mathrm{uL}$

Date Acquired : 05/03/2020 04:12:05 p. m.

Date Processed : 09/03/2020 04:43:05 p. m

Sample Type : Unknown

Acquired by : System Administrator

Processed by : : System Administrator

uV

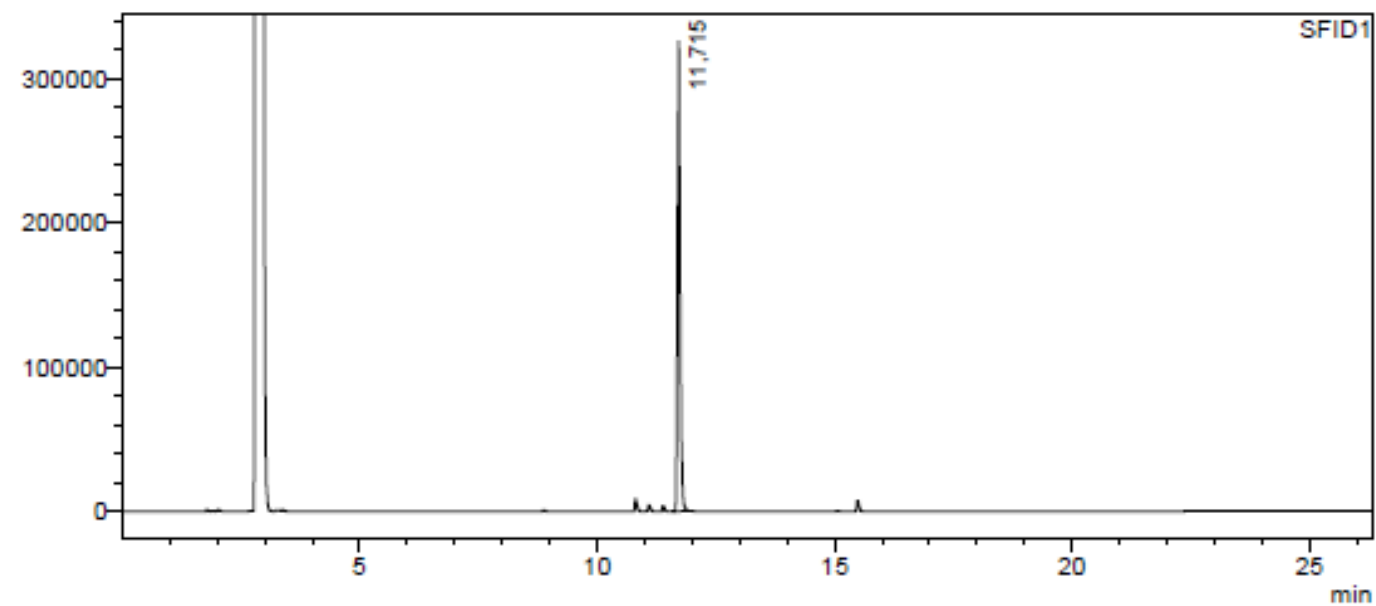

SFID

\begin{tabular}{|r|r|r|r|r|r|r|}
\hline Peak\# & Ret. Time & Area & Height & Area $\%$ & Conc. & Name \\
\hline 1 & 11,715 & 1236382 & 324213 & 100,000 & 100,000 & \\
\hline Total & & 1236382 & 324213 & 100,000 & & \\
\hline
\end{tabular}


Sample Name : SLS-172

Sample ID : SLS-172

Data Filename : $4 \mathrm{q}-\mathrm{SLS}-172 \mathrm{gcd}$

Method Filename : Method2-1min-eq.gcm

Batch Filename : 05032020.gcb

Vial \# : 3

Injection Volume : $1 \mathrm{uL}$

Date Acquired : 05/03/2020 11:53:29 a. m.

Sample Type : Unknown

Date Processed : 09/03/2020 04:13:21 p. m.

Acquired by : : System Administrator

Processed by : System Administrator

uV

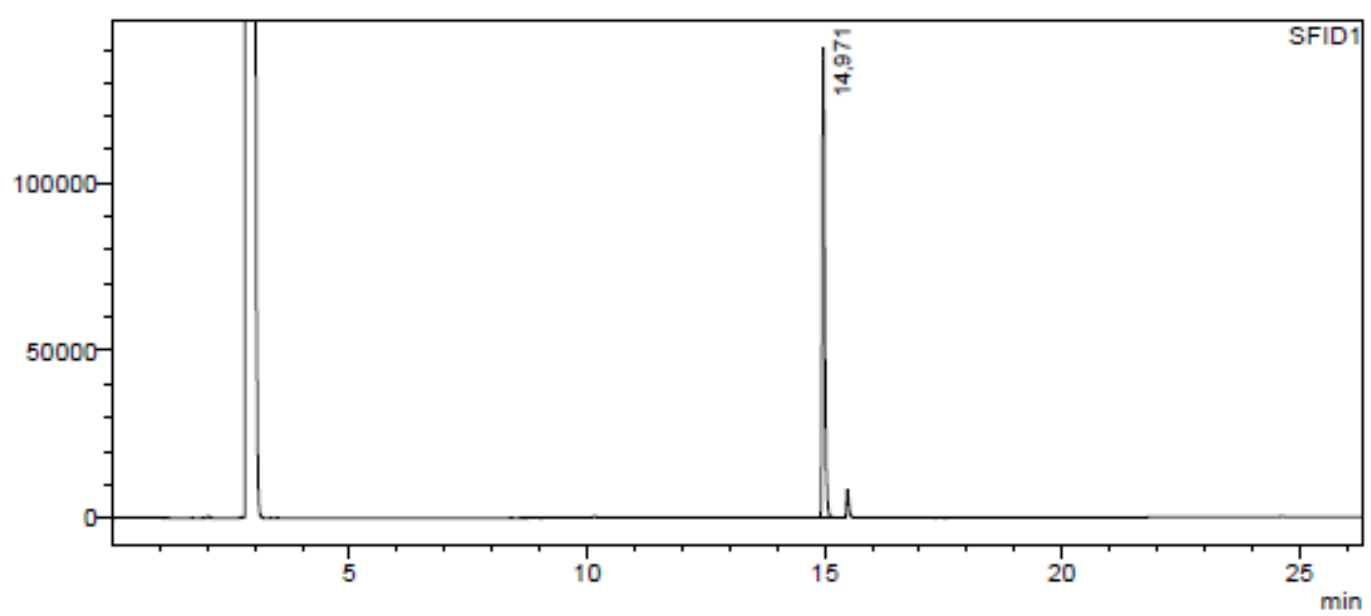

\begin{tabular}{|c|c|c|c|c|c|c|}
\hline Peak\# & Ret. Time & Area & Height & Area\% & Conc. & Name \\
\hline 1 & 14,971 & 490446 & 139399 & 100,000 & 100,000 & \\
\hline Total & & 490446 & 139399 & 100,000 & & \\
\hline
\end{tabular}


Analysis Date \& Time : $11 / 11 / 2016$ 08:11:36 a. m.

User Name : Admin

Vial\# $: 2$

$\begin{array}{ll}\text { Sample Name } & : 2 \\ \text { Sample ID } & : 2\end{array}$

Sample ID

Sample Type

Injection Volume

ISTD Amount

Unknown

: 1

Data Name : C:IUsers IShimadzu GCIDocuments Productos-JOC15a-1-phenylethanol.gcd

Method Name

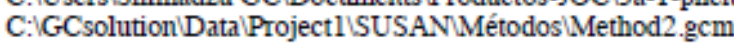

Intensity

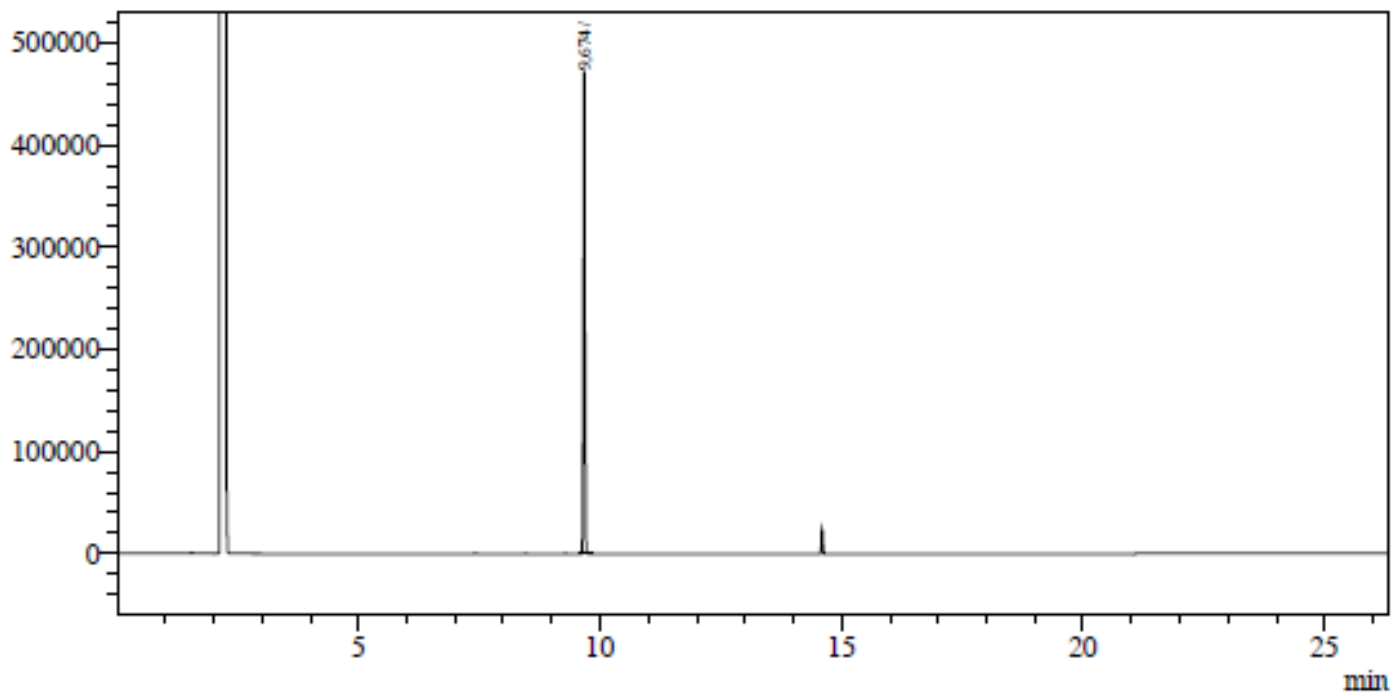

FID1

Pealk\# Ret.Time Area Height Conc. Unit Mark ID\# Cmpd Name

\begin{tabular}{crrrr}
1 & 9,674 & 998442 & 464783 & 0,000 \\
\hline Total & 998442 & 464783
\end{tabular}


Sample Name

Sample ID

Data Filename

Red-CG-01

Red-CG-01

5b-Red-CG-01.gcd

: Method2-1min-eq.gcm

Batch Filename : 03032020.gcb

Vial \# : 3

Injection Volume : 1 uL

Date Acquired : 03/03/2020 01:06:13 p. m.

Date Processed : 03/03/2020 01:32:37 p. m.
Sample Type : Unknown

Acquired by : : System Administrator

Processed by : System Administrator

uV

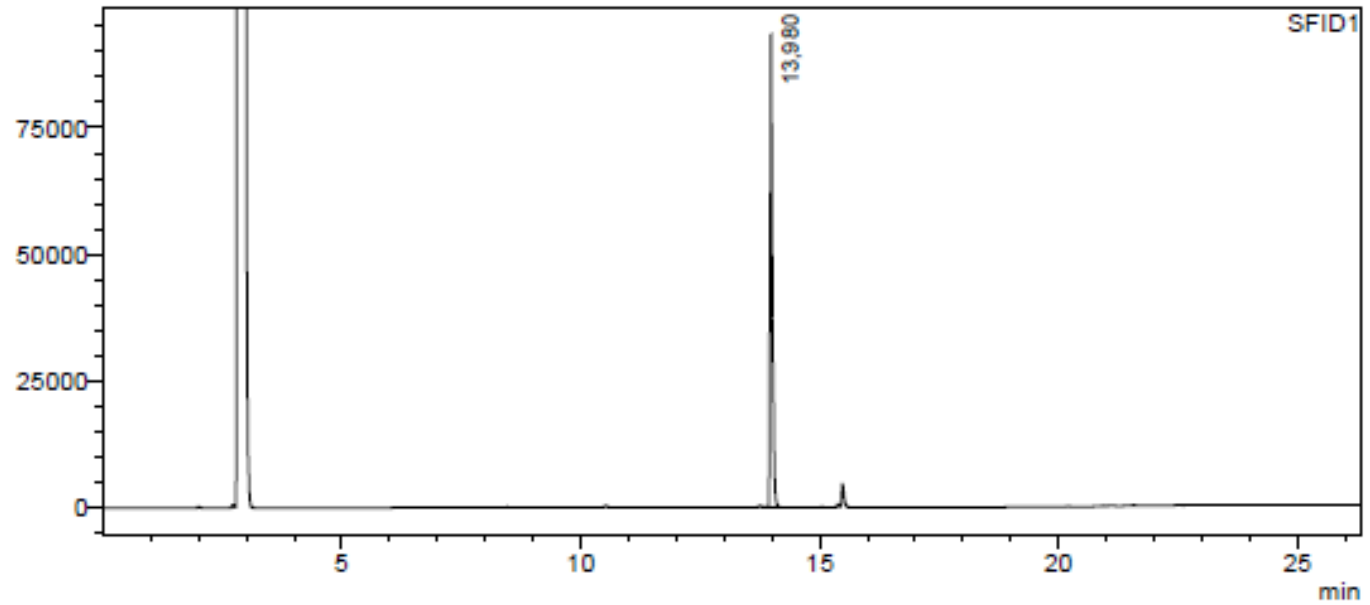

SFID1

\begin{tabular}{|r|r|r|r|r|r|r|}
\hline Peak\# & Ret. Time & Area & Height & Area\% & Conc. & Name \\
\hline 1 & 13,980 & 311780 & 92997 & 100,000 & 100,000 & \\
\hline Total & & 311780 & 92997 & 100,000 & & \\
\hline
\end{tabular}


Sample Name : :SLS-181

Sample ID : SLS-181

Data Filename : 5c-SLS-181.gcd

Method Filename : Method2-1min-eq.gcm

Batch Filename : 06032020.gcb

Vial \#

Injection Volume : 1 uL

Date Acquired $\quad: 06 / 03 / 202012: 44: 31$ p. m.

Sample Type : Unknown

Date Processed : 09/03/2020 04:51:26 p. m.

Acquired by : System Administrator

Processed by : System Administrator

uV

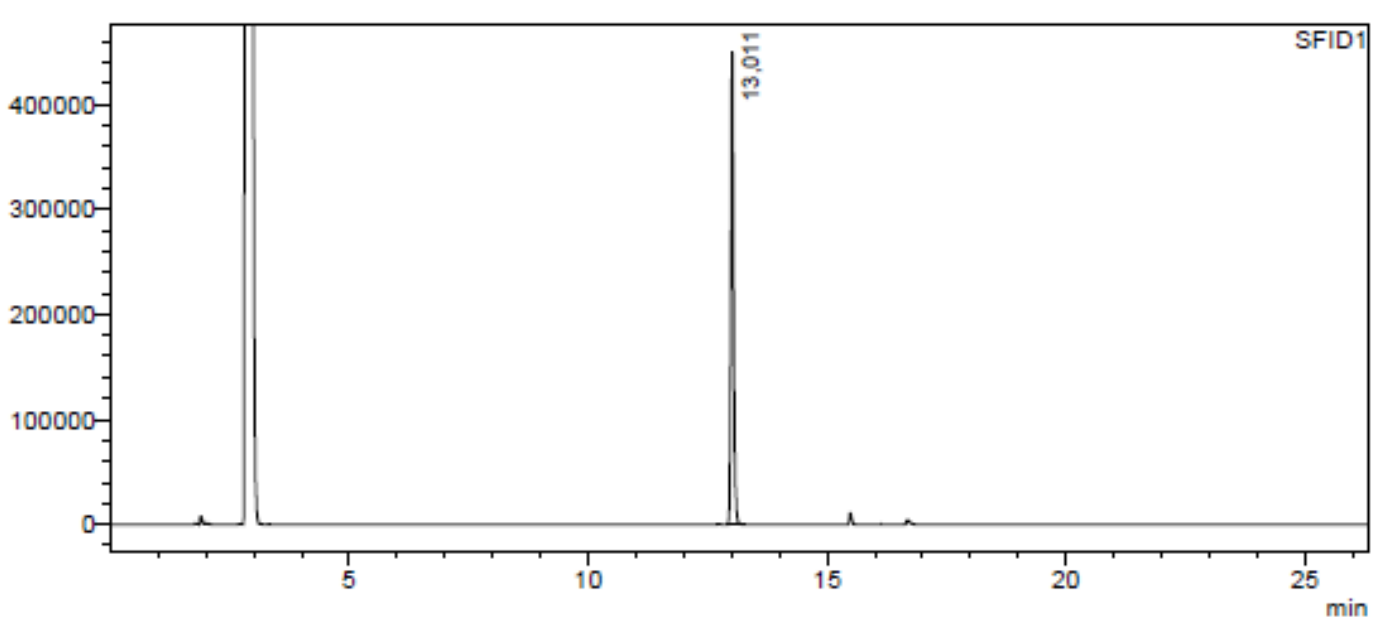

SFID1

\begin{tabular}{|r|r|r|r|r|r|r|}
\hline Peak\# & Ret. Time & \multicolumn{1}{|c|}{ Area } & Height & Area\% & \multicolumn{1}{c|}{ Conc. } & Name \\
\hline 1 & 13,011 & 1740294 & 445765 & 100,000 & 100,000 & \\
\hline Total & & 1740294 & 445765 & 100,000 & & \\
\hline
\end{tabular}


Sample Name

Sample ID

Data Filename

Method Filenam

Batch Filename

Vial \#

Injection Volume: :1

Date Acquired

Date Processed

- NC-Red-20

NC-Red-20

5d-NC-Red-20.gcd

Method2-1min-eq.gcm

$03032020 . \mathrm{gcb}$

2

03/03/2020 12:26:27 p. m.

: 03/03/2020 12:52:49 p. m.
Sample Type : Unknown

Acquired by : : System Administrator Processed by : System Administrator

uV

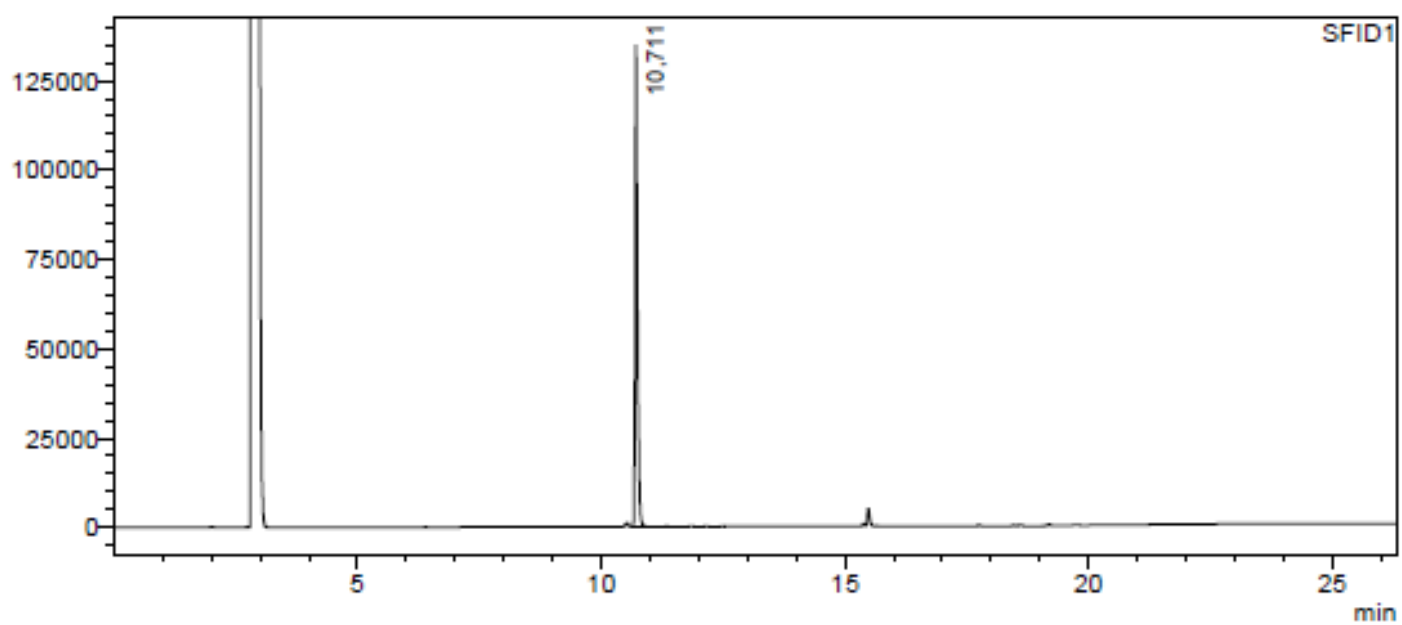

SFID1

\begin{tabular}{|r|r|r|r|r|r|r|}
\hline Peak\# & Ret. Time & \multicolumn{1}{|c|}{ Area } & \multicolumn{1}{c|}{ Height } & \multicolumn{1}{c|}{ Area\% } & Conc. & Name \\
\hline 1 & 10,711 & 449051 & 133652 & 100,000 & 100,000 & \\
\hline Tota & & 449051 & 133652 & 100,000 & & \\
\hline
\end{tabular}


Sample Name : SLS-194

Sample ID : SLS-194

Data Filename : 5e-SLS-194.gcd

Method Filename : Method2-1min-eq.gcm

Batch Filename : 10032020.gcb

Vial \# : 3

Injection Volume : $1 \mathrm{uL}$

Date Acquired $\quad: 10 / 03 / 202012: 31: 46$ p. m.

Date Processed : : 10/03/2020 12:58:09 p. m.

Sample Type : Unknown

Acquired by : System Administrator

Processed by : :System Administrator

uV

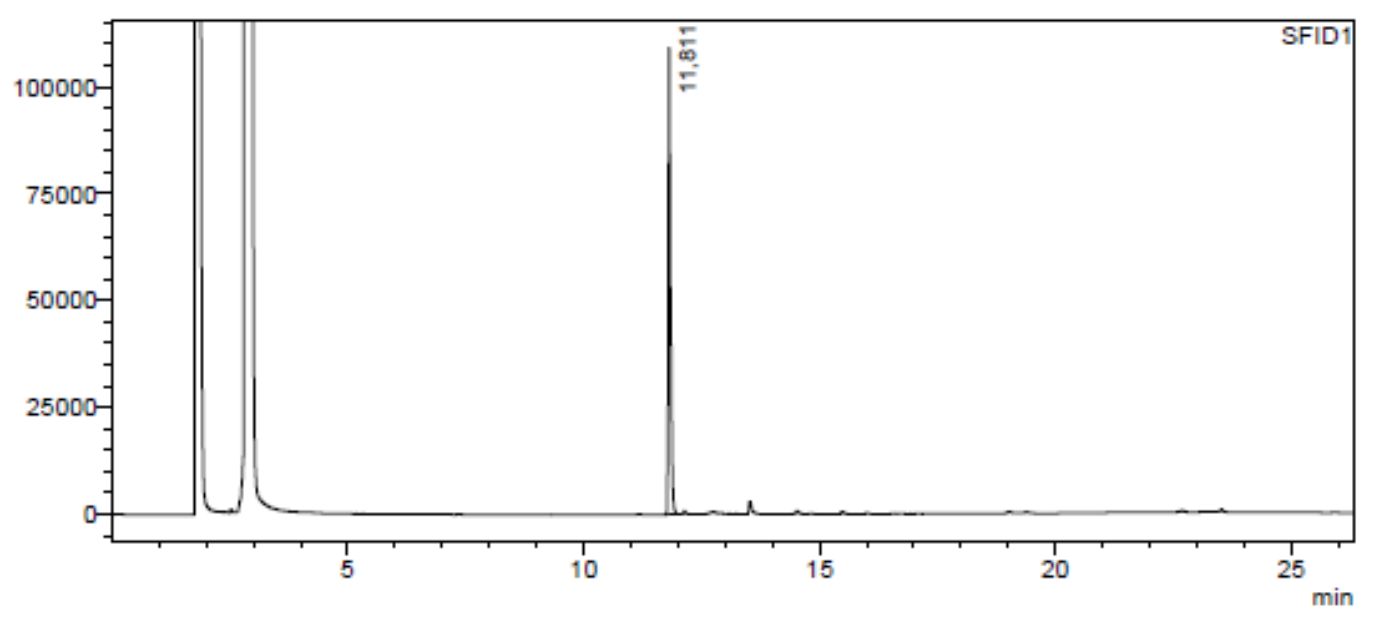

\begin{tabular}{|c|c|c|c|c|c|c|}
\hline Peak\# & Ret. Time & Area & Height & Area $\%$ & Conc. & Name \\
\hline 1 & 11,811 & 362845 & 108695 & 100,000 & 100,000 & \\
\hline Total & & 362845 & 108695 & 100,000 & & \\
\hline
\end{tabular}


Sample Name : SLS-183-2

Sample ID : : SLS-183-2

Data Filename : 5f-SLS-183-2.gcd

Method Filename : Method2-1min-eq.gcm

Batch Filename

Vial \#

$: 6$

Injection Volume : $1 \mathrm{uL}$

Date Acquired : : 11/03/2020 11:38:10 a. m.

Sample Type : Unknown

Date Processed : :11/03/2020 12:06:37 p. m.

Acquired by : System Administrator Processed by : System Administrator

uV

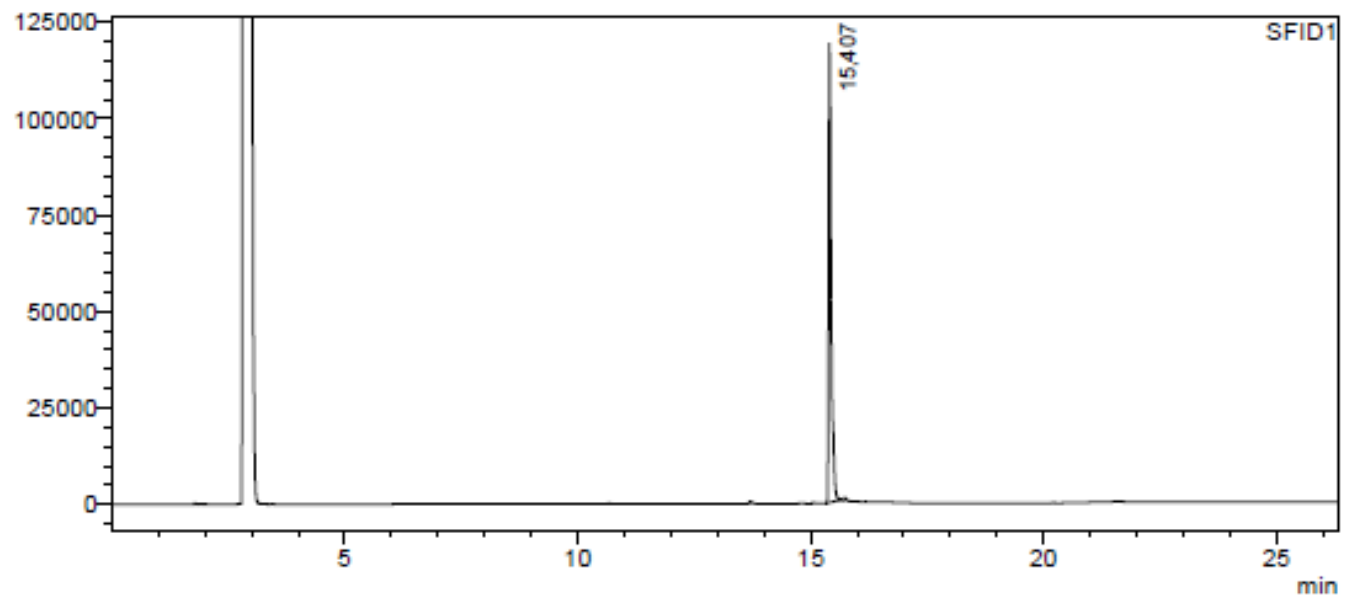

SFID1

\begin{tabular}{|r|r|r|r|r|r|r|}
\hline Peak\# & Ret. Time & \multicolumn{1}{|c|}{ Area } & Height & Area\% & Conc. & Name \\
\hline 1 & 15,407 & 448714 & 118528 & 100,000 & 100,000 & \\
\hline Total & & 448714 & 118528 & 100,000 & & \\
\hline
\end{tabular}


Sample Name : SLS-184

Sample ID : : SLS-184

Data Filename : $5 i-S L S-184 . g c d$

Method Filename : Method2-1min-eq.gcm

Batch Filename :06032020.gcb

Vial \#

Injection Volume : 1 ul

Date Acquired :06/03/2020 03:01:07 p. m.

Sample Type : Unknown

Date Processed : 09/03/2020 05:00:59 p. m.

Acquired by : System Administrator

Processed by : System Administrator

uV

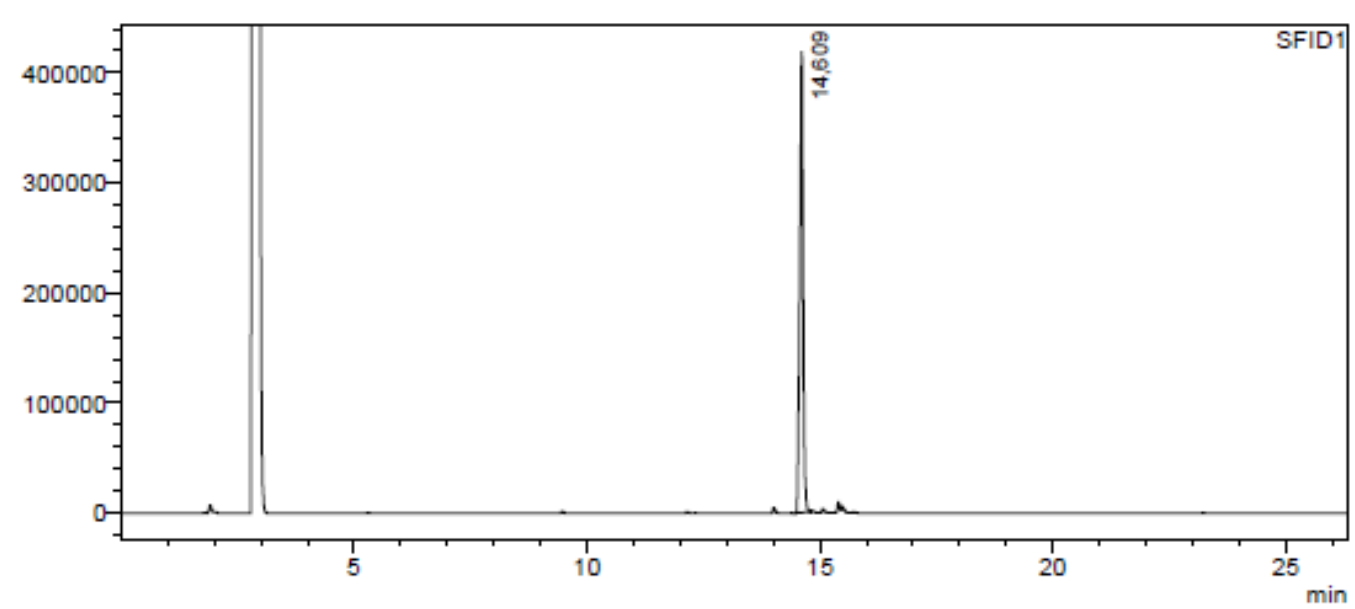

SFID

\begin{tabular}{|r|r|r|r|r|r|r|}
\hline Peak\# & Ret. Time & \multicolumn{1}{|c|}{ Area } & Height & Area $\%$ & \multicolumn{1}{c|}{ Conc. } & Name \\
\hline 1 & 14,609 & 2048484 & 417295 & 100,000 & 100,000 & \\
\hline Total & & 2048484 & 417295 & 100,000 & & \\
\hline
\end{tabular}


Sample Name : SLS-187

Sample ID : : SLS-187

Data Filename : 5 j-SLS-187.gcd

Method Filename : Method2-1min-eq.gcm

Batch Filename : $06032020 . \mathrm{gcb}$

Vial \#

5

Injection Volume : 1 uL

Date Acquired $\quad: 06 / 03 / 2020$ 05:18:42 p. m.

Sample Type : Unknown

Date Processed : 09/03/2020 05:21:41 p. m.

Acquired by : : System Administrator

Processed by : System Administrator

uV

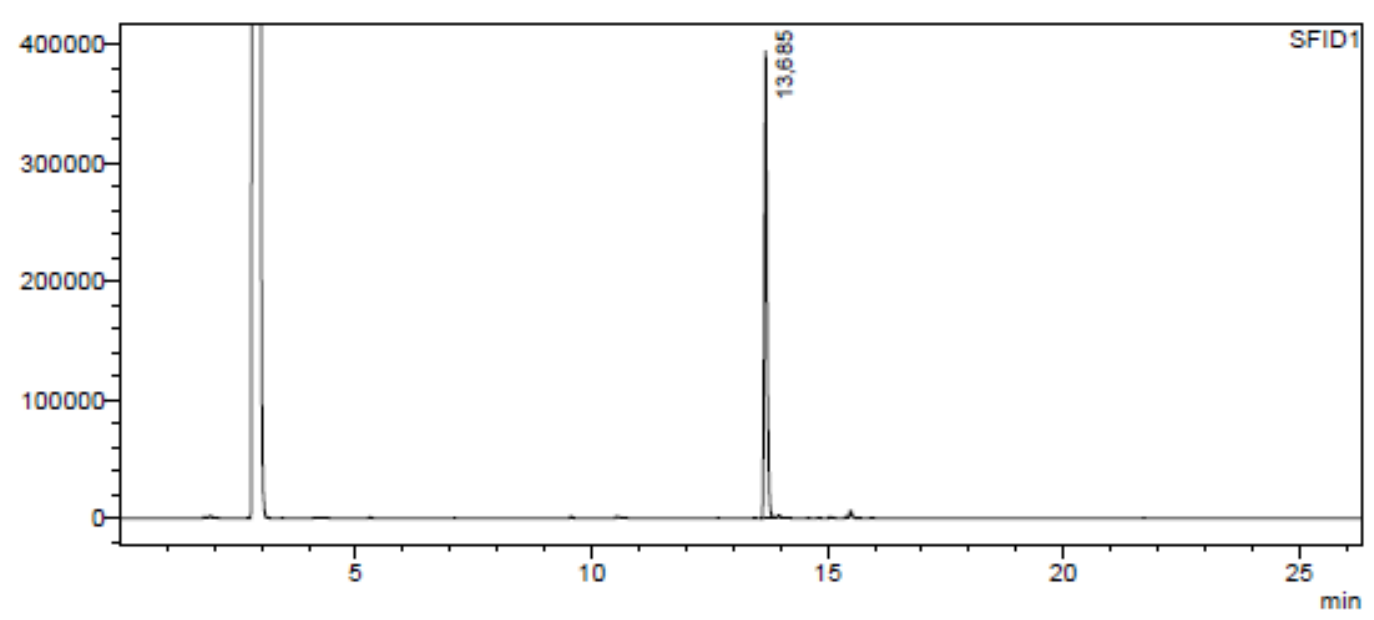

\begin{tabular}{|c|c|c|c|c|c|c|}
\hline Peak\# & Ret. Time & Area & Height & Area\% & Conc. & Name \\
\hline 1 & 13,685 & 1497371 & 393942 & 100,000 & 100,000 & \\
\hline Total & & 1497371 & 393942 & 100,000 & & \\
\hline
\end{tabular}


Sample Name : SLS-186

Sample ID : SLS-186

Data Filename : $5 \mathrm{k}-\mathrm{SLS}-186 . \mathrm{gcd}$

Method Filename : Method2-1min-eq.gcm

Batch Filename : $06032020 . \mathrm{gcb}$

Vial \#

Injection Volume : $1 \mathrm{uL}$

Date Acquired : :06/03/2020 04:33:56 p. m.

Date Processed $\quad$ : 09/03/2020 05:19:35 p. m.

Sample Type : Unknown

Acquired by : System Administrator Processed by : System Administrator

uV

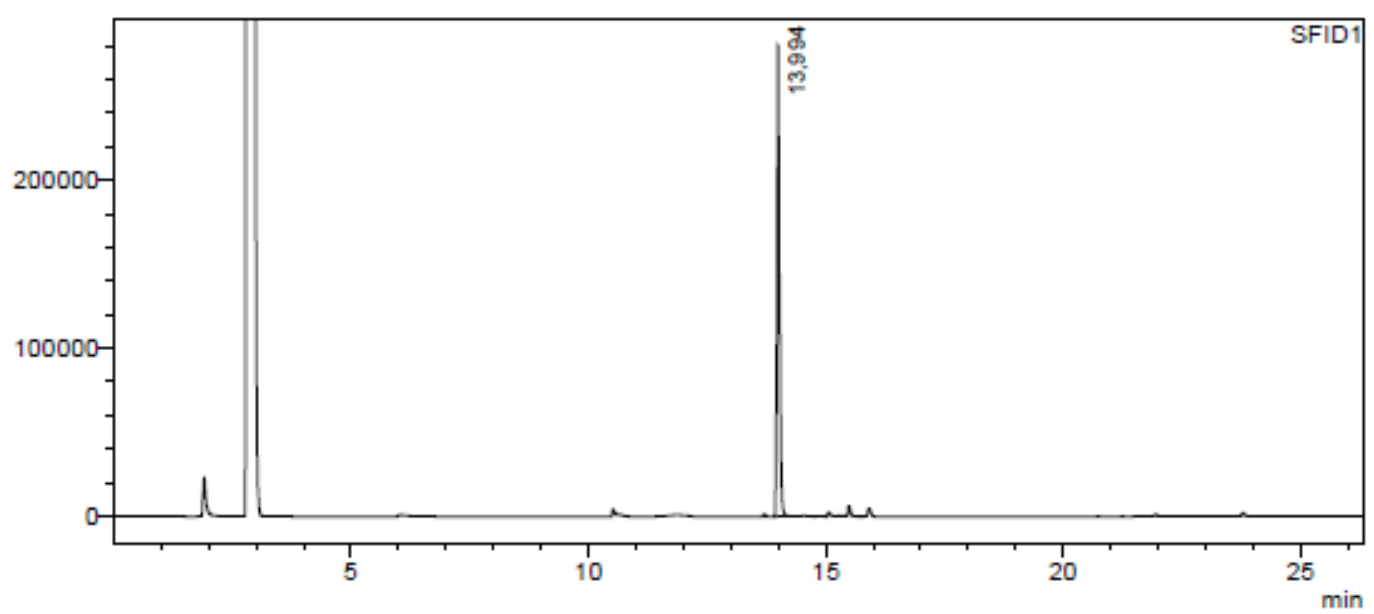

\begin{tabular}{|c|c|c|c|c|c|c|}
\hline Peak\# & Ret. Time & Area & Height & Area $\%$ & Conc. & Name \\
\hline 1 & 13,994 & 1023519 & 279690 & 100,000 & 100,000 & \\
\hline Total & & 1023519 & 279690 & 100,000 & & \\
\hline
\end{tabular}


Sample Name : NC-Red-26

Sample ID

Data Filename

NC-Red-26

-5m-NC-Red-26.gcd

Method Filename : Method2-1min-eq.gcm

Batch Filename : $03032020 . \mathrm{gcb}$

Vial \# : : 1

njection Volume : : 1 uL

Date Acquired : 03/03/2020 11:47:52 a. m.

Date Processed : :03/03/2020 12:14:13 p. m.
Sample Type : Unknown

Acquired by : : System Administrator Processed by : System Administrator

uV

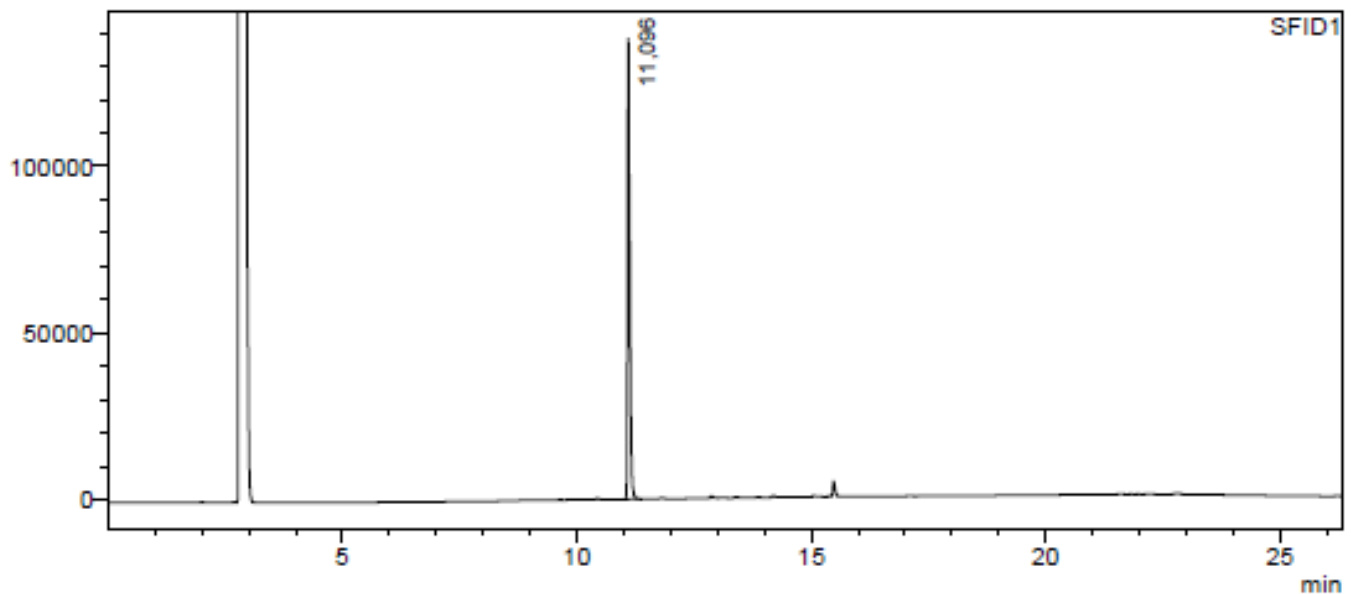

SFID1

\begin{tabular}{|r|r|r|r|r|r|r|}
\hline Peak\# & Ret. Time & \multicolumn{1}{|c|}{ Area } & Height & \multicolumn{1}{c|}{ Area $\%$} & Conc. & Name \\
\hline 1 & 11,096 & 457903 & 137871 & 100,000 & 100,000 & \\
\hline Total & & 457903 & 137871 & 100,000 & &
\end{tabular}


Sample Name : SLS-193-2

Sample ID

: SLS-193-2

Data Filename : $5 n-S L S-193-2 . g c d$

Method Filename : Method2-1min-eq.gcm

Batch Filename

Vial \#

1

Injection Volume : $1 \mathrm{uL}$

Date Acquired : :11/03/2020 12:25:45 p. m.

Sample Type : Unknown

Date Processed : : 11/03/2020 01:34:18 p. m.

Acquired by : System Administrator

Processed by : System Administrator

uV

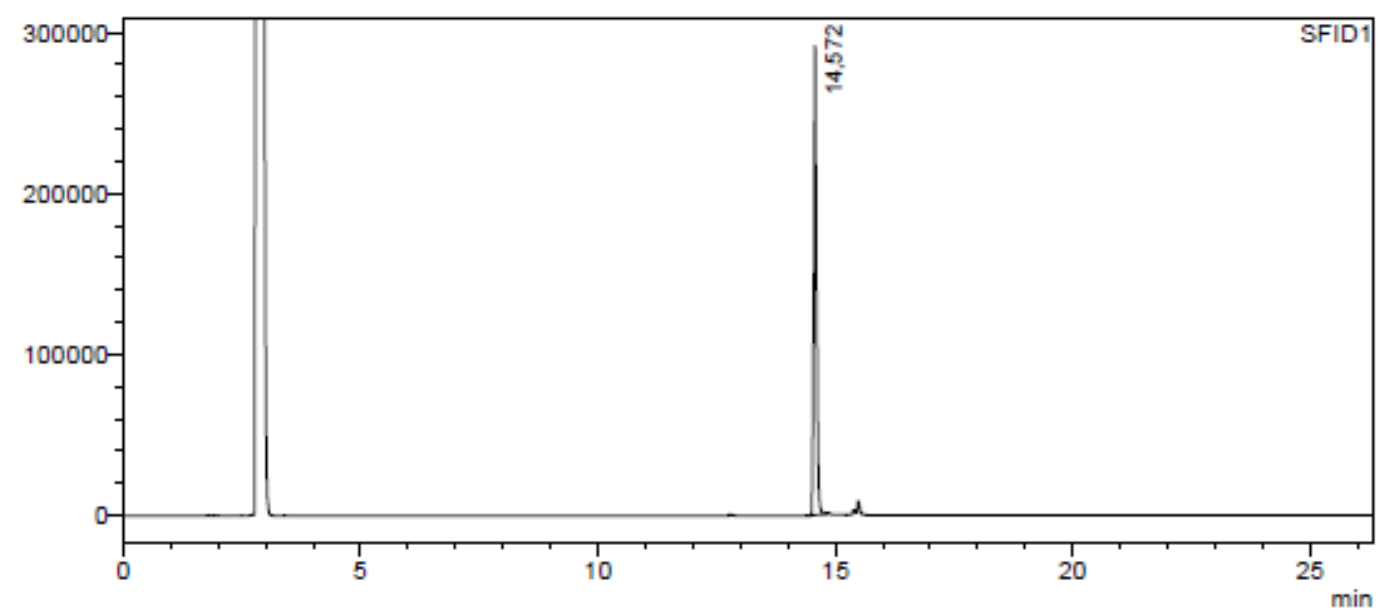

SFID1

\begin{tabular}{|r|r|r|r|r|r|r|}
\hline Peak\#\# & Ret. Time & Area & Height & Area\% & Conc. & Name \\
\hline 1 & 14,572 & 1145778 & 290898 & 100,000 & 100,000 & \\
\hline Total & & 1145778 & 290898 & 100,000 & & \\
\hline
\end{tabular}




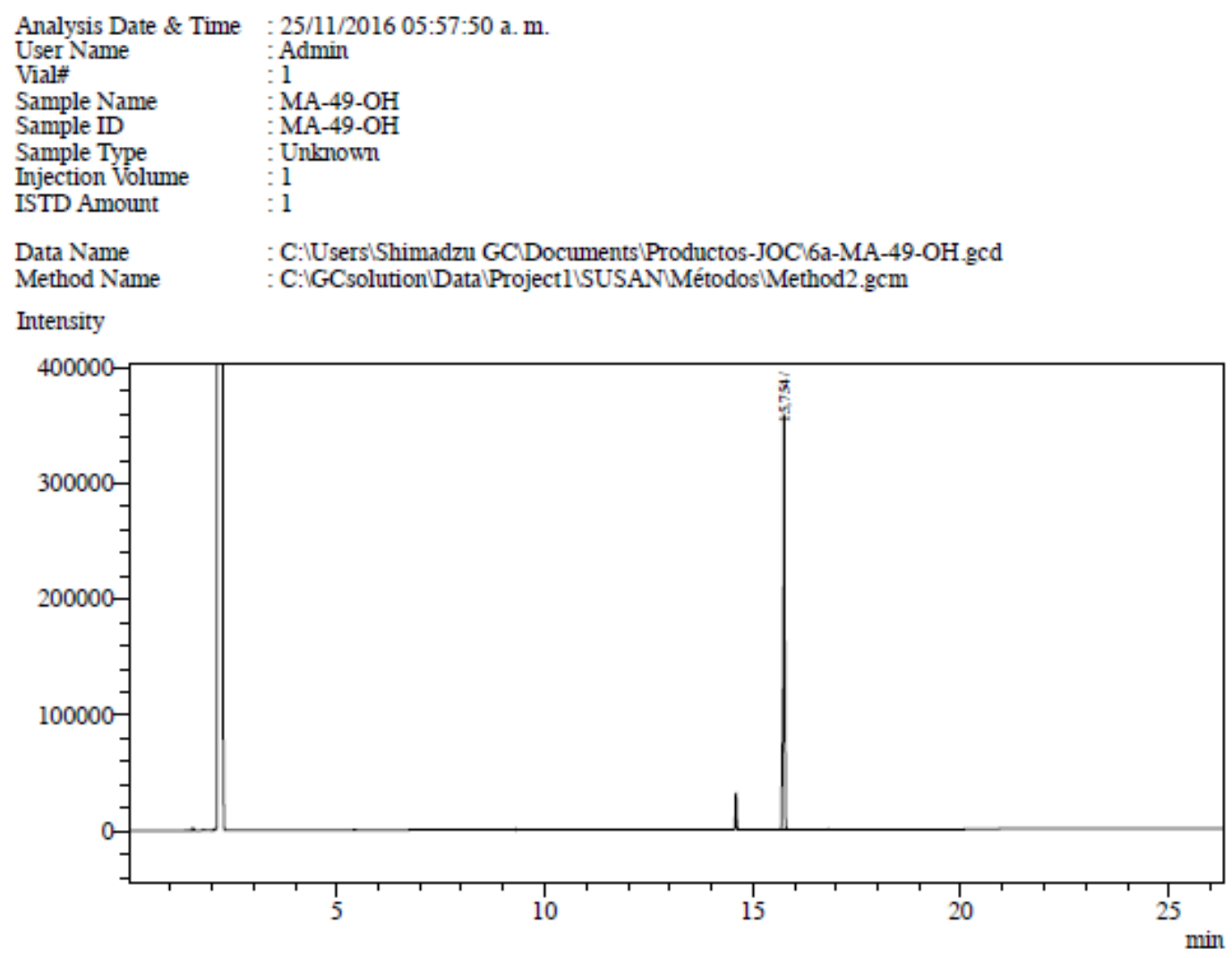

FID1

\begin{tabular}{|c|c|c|c|c|c|}
\hline $\begin{array}{l}\text { als:\# Ret.Time } \\
15,754\end{array}$ & $\begin{array}{l}\text { Area } \\
848977\end{array}$ & $\begin{array}{l}\text { Height } \\
354150\end{array}$ & $\begin{array}{l}\text { Conc. } \\
0,000\end{array}$ & Unit Mark ID\# & Cmpd Name \\
\hline
\end{tabular}

$\begin{array}{llll}\text { Total } & 848977 & 354150\end{array}$


Sample Name : : SLS-191-4

Sample ID : : SLS-191-4

Data Filename : 6b-SLS-191-4.gcd

Method Filename : Method2-1min-eq.gcm

Batch Filename :

Vial \#

4

Injection Volume : $1 \mathrm{uL}$

Date Acquired : : 11/03/2020 04:41:07 p. m.

Date Processed $\quad: 11 / 03 / 2020$ 05:07:30 p. m.

Sample Type : Unknown

Acquired by : System Administrator

Processed by : System Administrator

uV

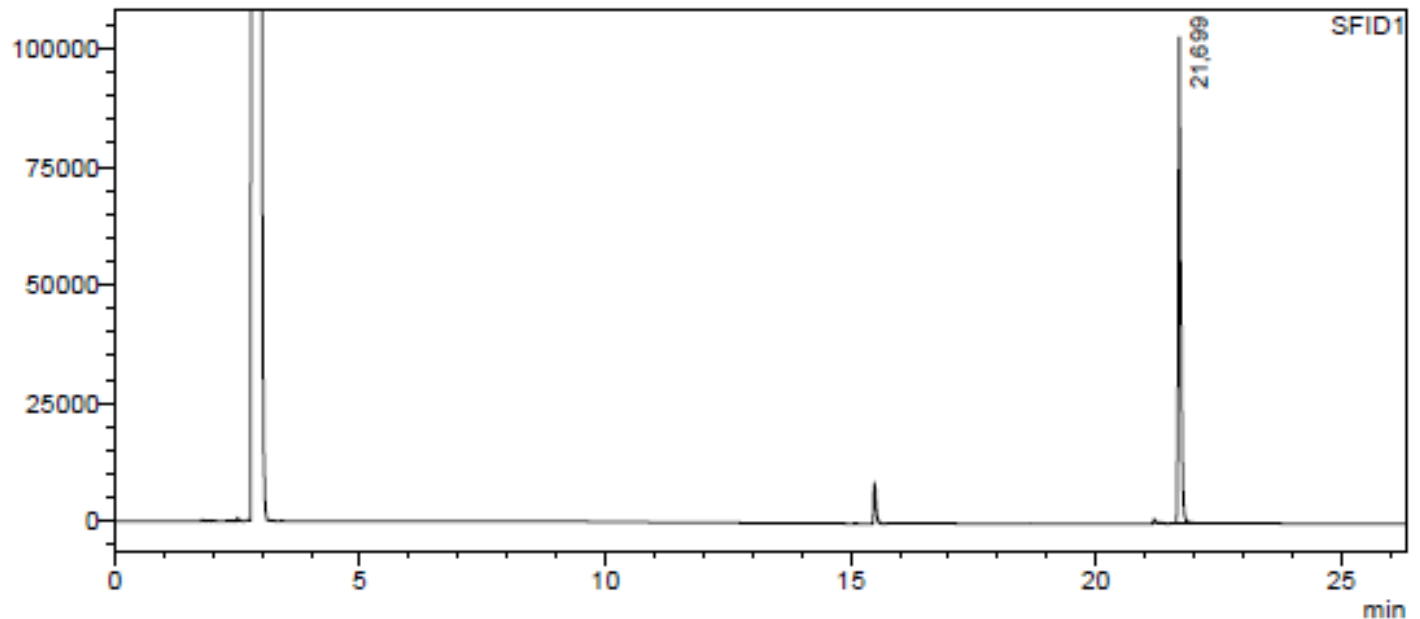

SFID1

\begin{tabular}{|r|r|r|r|r|r|r|}
\hline Peak\# & Ret. Time & \multicolumn{1}{c|}{ Area } & Height & \multicolumn{1}{c|}{ Area $\%$} & \multicolumn{1}{c|}{ Conc. } & Name \\
\hline 1 & 21,699 & 367038 & 102434 & 100,000 & 100,000 & \\
\hline Total & & 367038 & 102434 & 100,000 & & \\
\hline
\end{tabular}




\section{Sample Name : MA-85}

Sample ID

MA-85

Data Filename

6c-MA

Method Filename : Method2-1min-eq.gcm

Batch Filename : 03032020.gcb

Vial \# : 4

Injection Volume : $1 \mathrm{uL}$

Date Acquired : 03/03/2020 01:47:08 p. m

Sample Type : Unknown

Date Processed : :03/03/2020 02:13:31 p. m.

Acquired by : : System Administrator

Processed by : System Administrator

uV

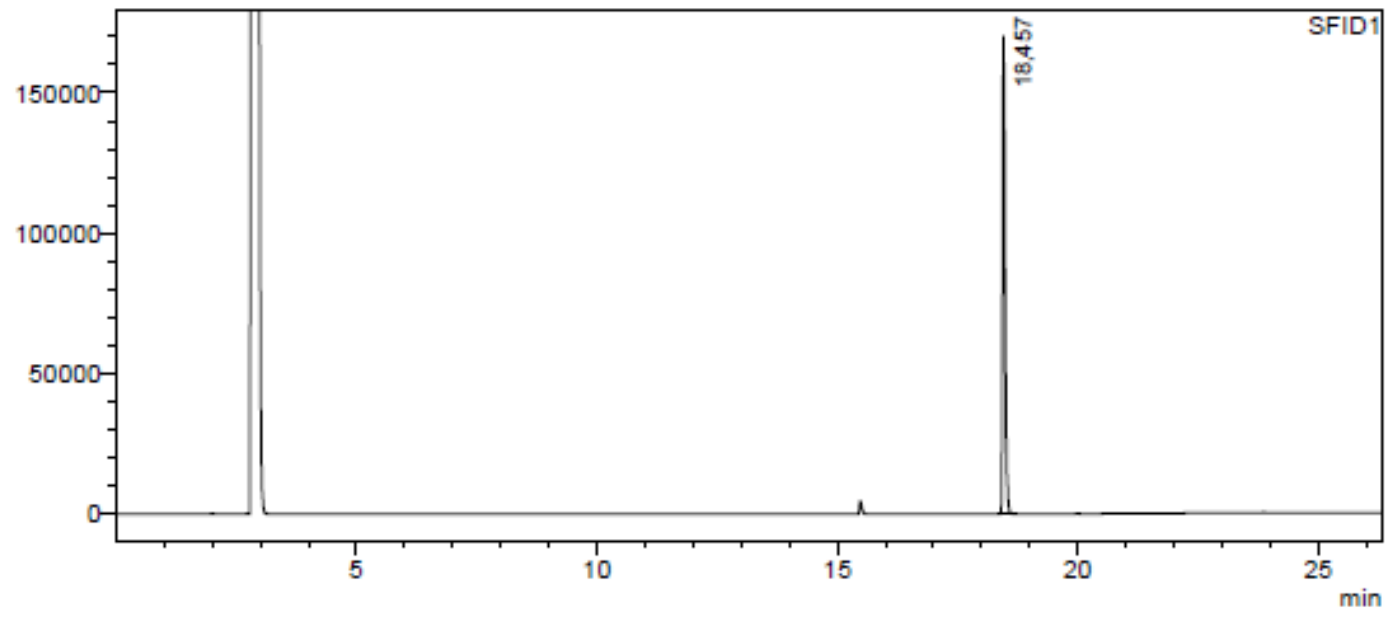

\begin{tabular}{|c|c|c|c|c|c|c|}
\hline Peak\# & Ret. Time & Area & Height & Area $\%$ & Conc. & Name \\
\hline 1 & 18,457 & 586706 & 168312 & 100,000 & 100,000 & \\
\hline Total & & 586706 & 168312 & 100,000 & & \\
\hline
\end{tabular}


Sample Name : MA-86

Sample ID : MA-86

Data Filename : 6d-MA-86.gcd

Method Filename : Method2-1min-eq.gcm

Batch Filename :03032020.gcb

Vial \#

njection Volume : $1 \mathrm{uL}$

Date Acquired : 03/03/2020 02:29:24 p. m

Date Processed $\quad: 03 / 03 / 2020$ 02:55:47 p. m.
Sample Type : Unknown

Acquired by : : System Administrator Processed by : System Administrator

uV

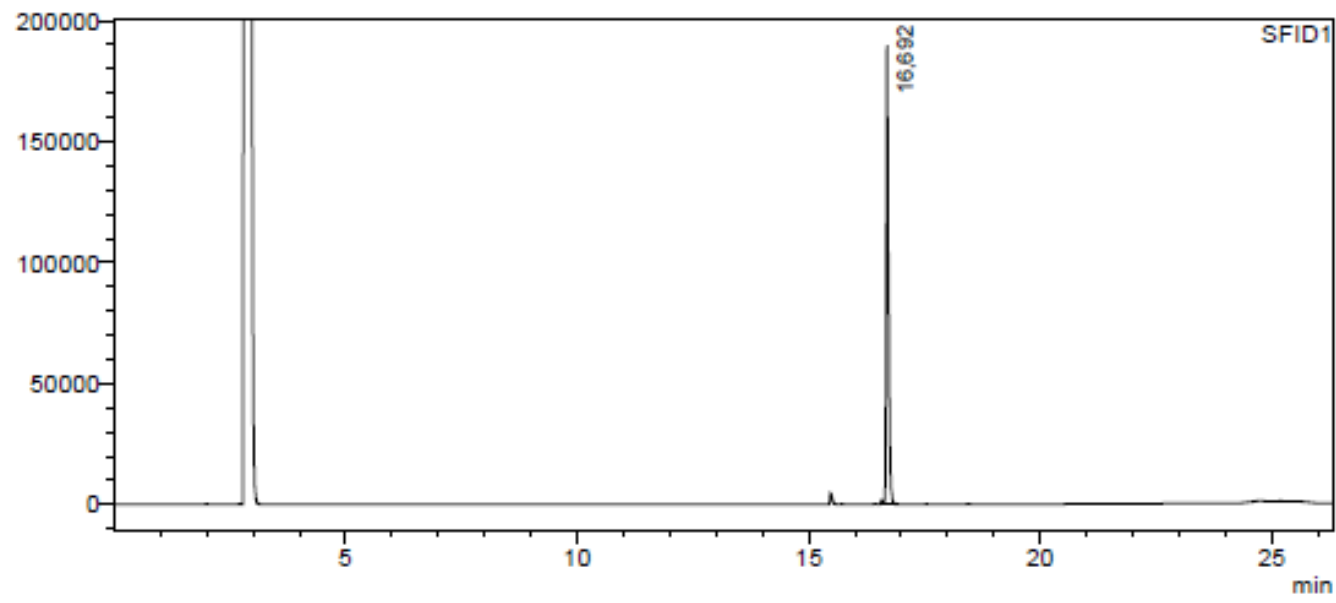

SFID1

\begin{tabular}{|r|r|r|r|r|r|r|}
\hline Peak\# & Ret. Time & Area & Height & Area $\%$ & Conc. & Name \\
\hline 1 & 16,692 & 646931 & 189026 & 100,000 & 100,000 & \\
\hline Total & & 646931 & 189026 & 100,000 & & \\
\hline
\end{tabular}


Print Date: 10 Mar 2020 08:00:14

MS Data Review Active Chromatogram and Spectrum Plots - 2020-03-10 08:00 AM

File: c: brukerwsimarcelol02012019inc red 26083xms

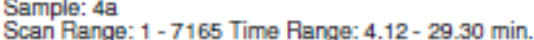

Operator: Marcelo

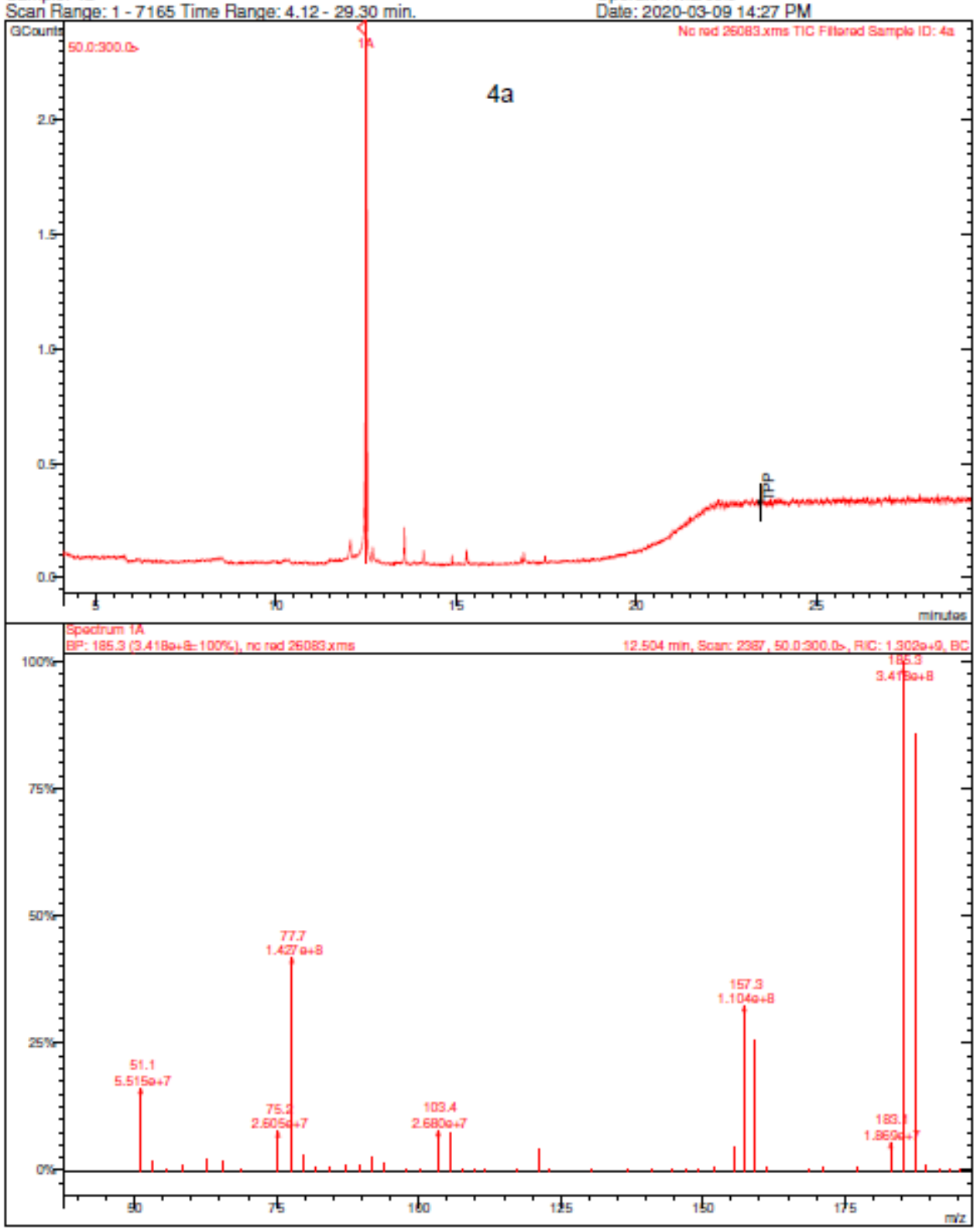


Print Date: 09 Mar 2020 10:20:28

MS Data Review Active Chromatogram and Spectrum Plots - 2020-03-09 10:20 AM

File: c:lbrukerwsimarcelol02012019inc red 26059xms Sample: $4 \mathrm{~b}$

Operator: Marcelo

Scan Range: 1 - 6371 Time Range: 4.13 - 26.51 min

Oate: $2020-03-06$ 18:48 PM

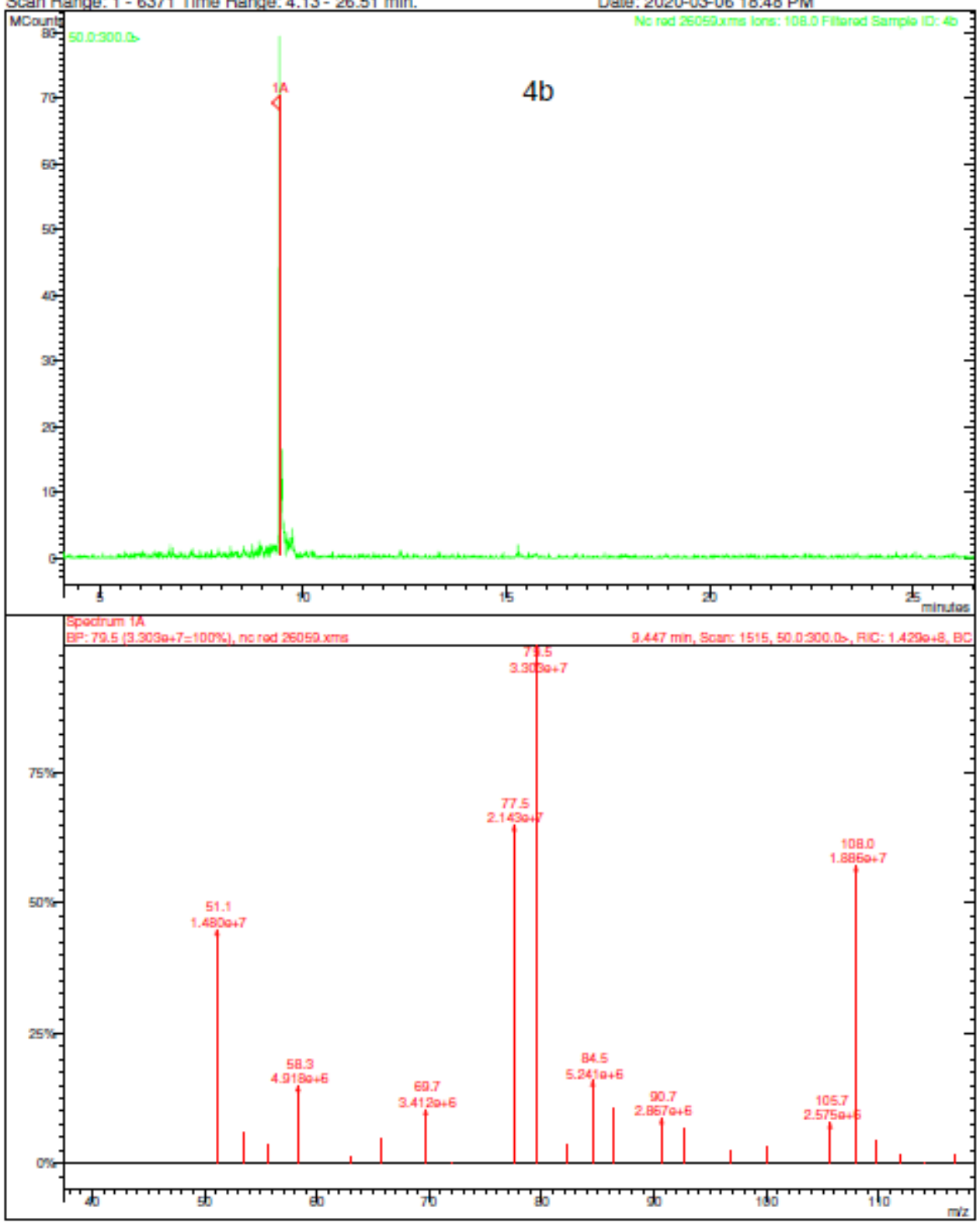


Print Date: 06 Mar 2020 10:45:18

MS Data Review Active Chromatogram and Spectrum Plots - 2020-03-06 10:45 AM

File: c:lbrukerwsimarcelolo2012019inc red 26029 xms

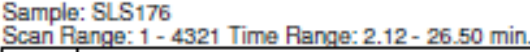

Operator: Marcelo

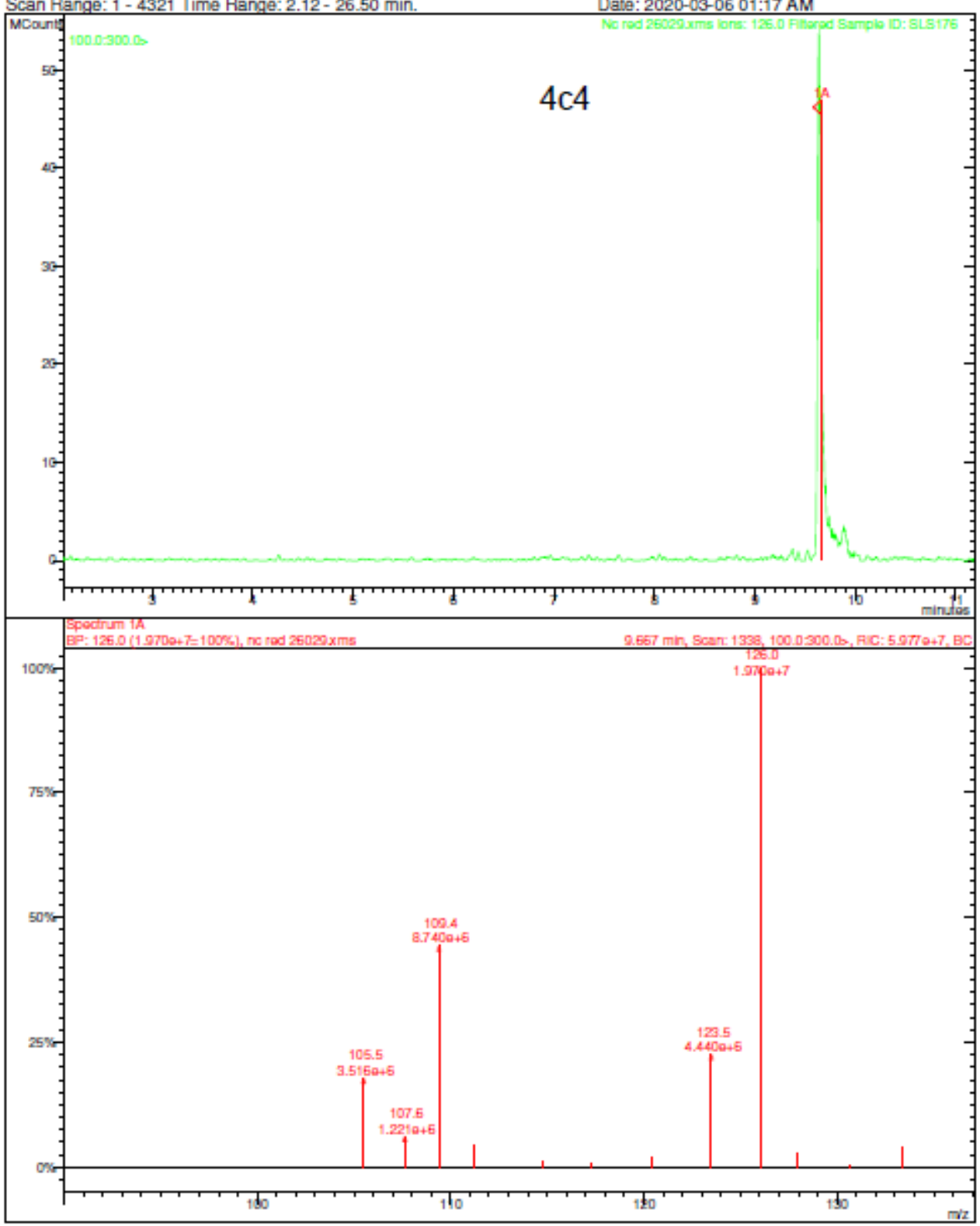


Print Date: 09 Mar 2020 14:16:44

MS Data Review Active Chromatogram and Spectrum Plots - 2020-03-09 14:16 PM

File: c:lbrukerwsimarcelol02012019inc red 26080 xms

Sample: acetone

Operator: Marcelo

Scan Range: 1 - 7171 Time Range: 4.12 - 29.31 min

Opera: 2020-03-09 12:32 PM

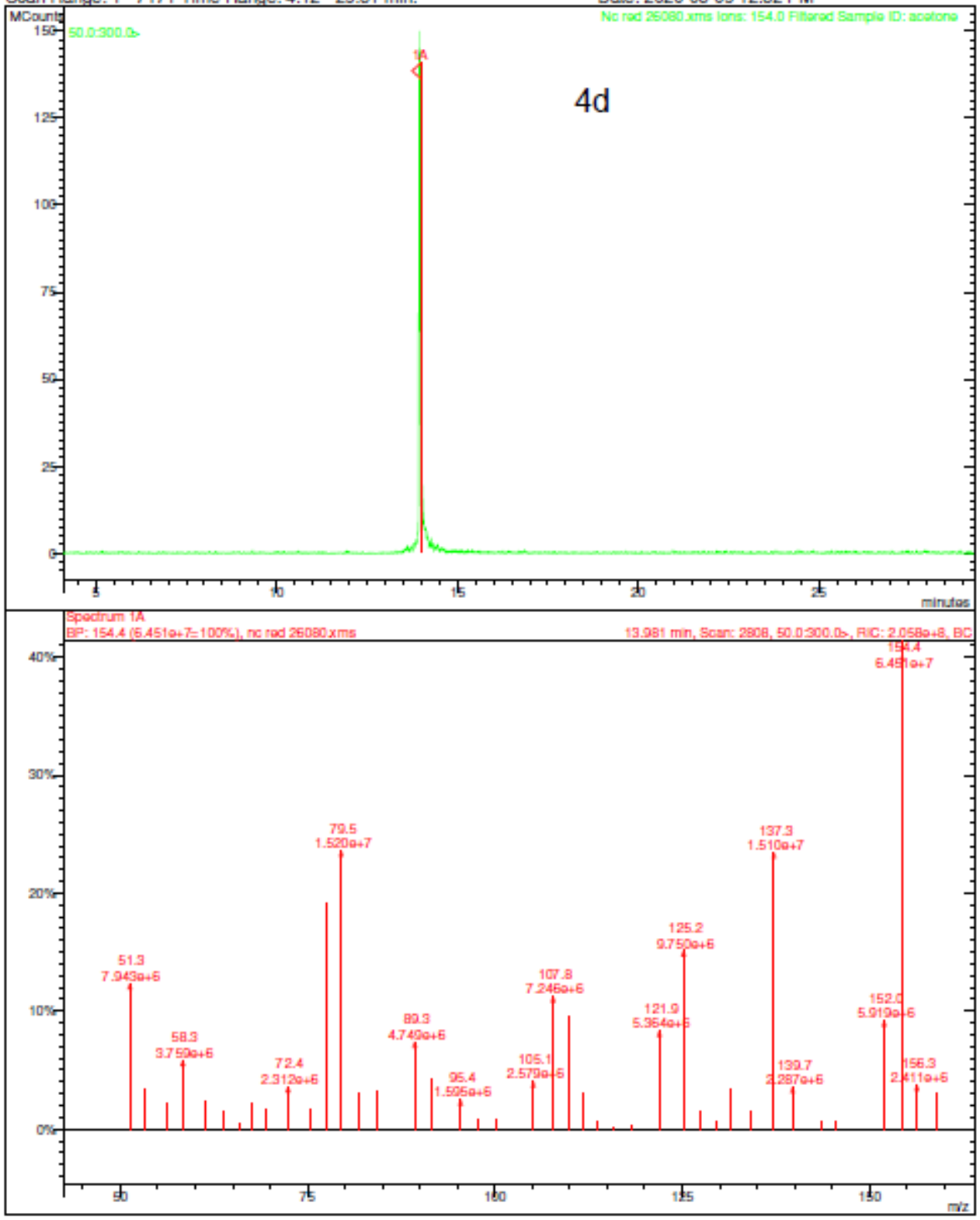


Print Date: 13 Mar 2020 11:08:31

MS Data Review Active Chromatogram and Spectrum Plots - 2020-03-13 11:08 AM

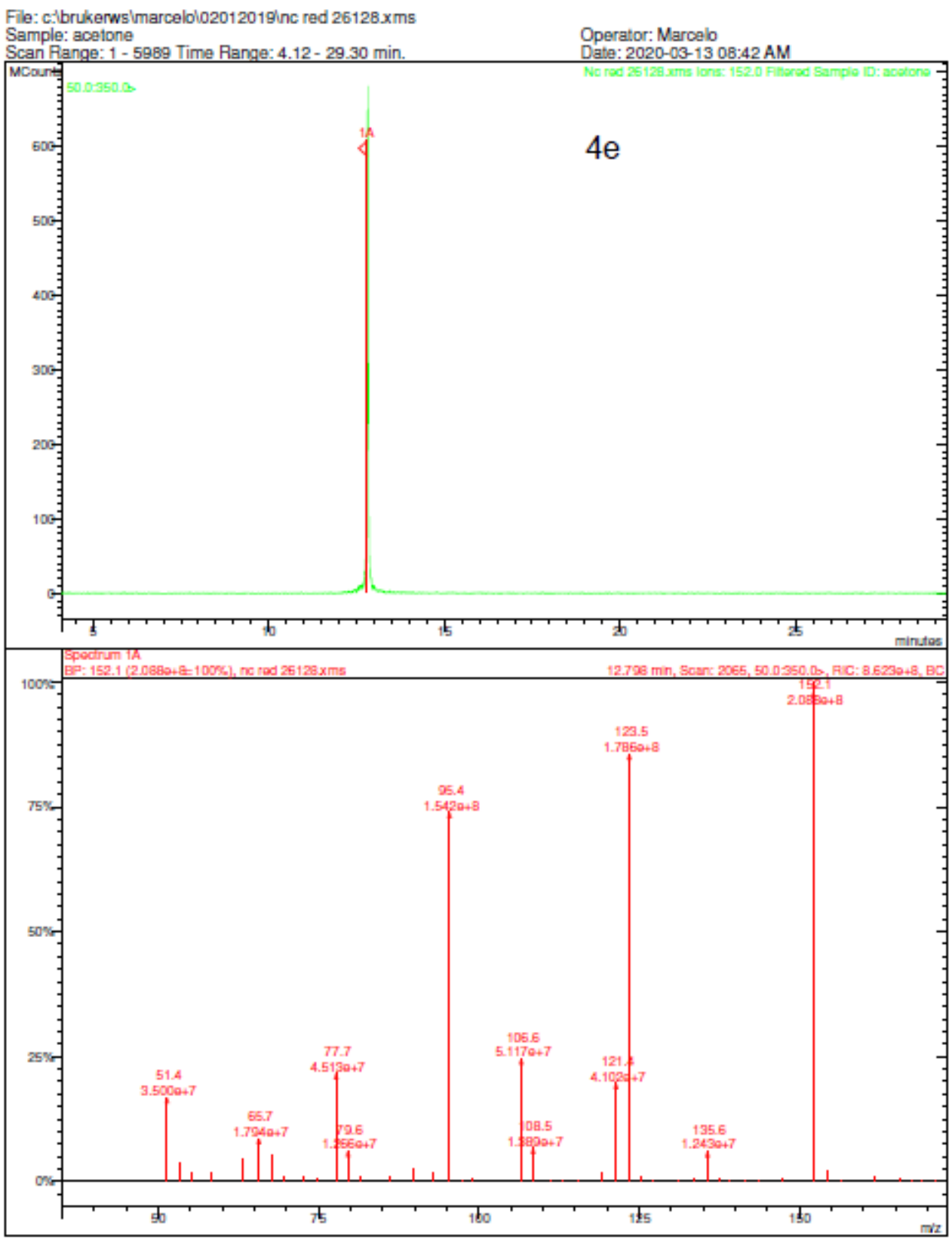


Print Date: 06 Mar 2020 11:01:24

MS Data Review Active Chromatogram and Spectrum Plots - 2020-03-06 11:01 AM

File: c.lbrukerwsimarcelolo2012019inc red 26036xms

Sample: acatone

Operator: Marcelo

Scan Range: 1 - 4321 Time Range: 2.12 - 26.50 min.

Opto: $2020-03-05$ 05:21 AM

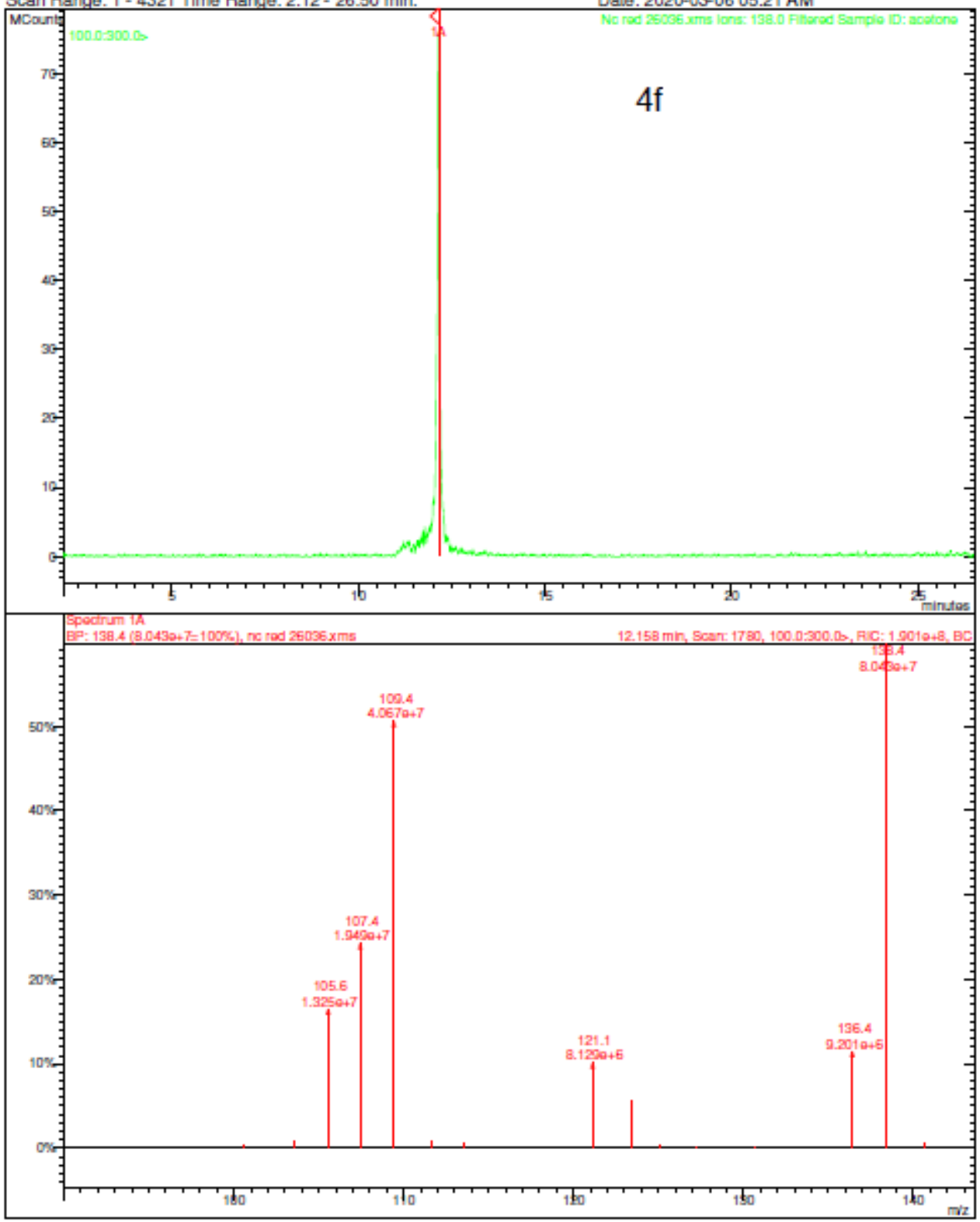


Print Date: 06 Mar 2020 08:28:44

MS Data Review Active Chromatogram and Spectrum Plots - 2020-03-06 08:28 AM

File: c:lbrukerwsimarcelolo2012019inc red 26020xms

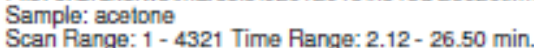

Operator: Marcelo

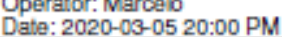

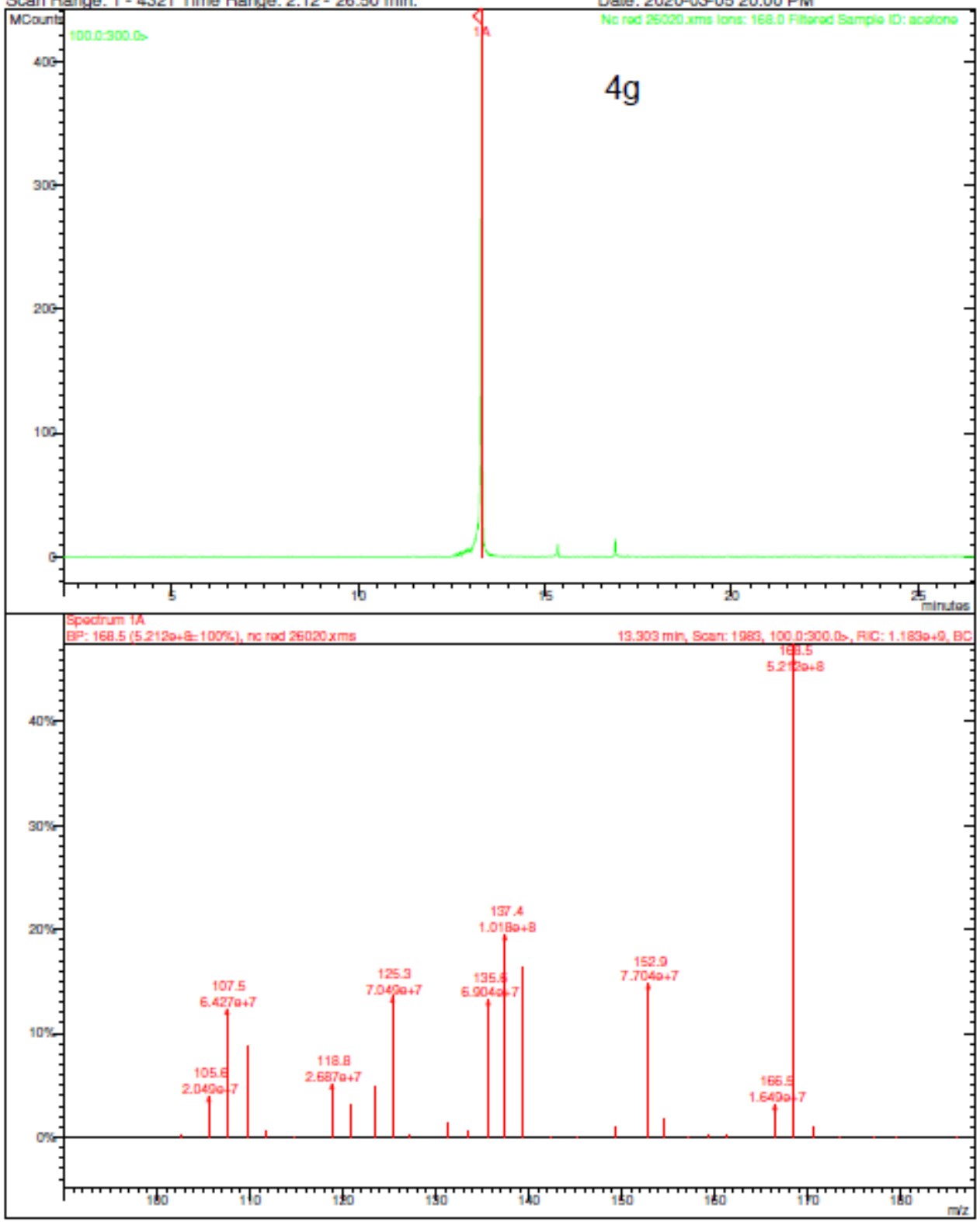


Print Date: 13 Mar 2020 07:57:33

MS Data Review Active Chromatogram and Spectrum Plots - 2020-03-13 07:57 AM

File: c:lbrukerwsimarcelolo2012019inc red 26121 xms

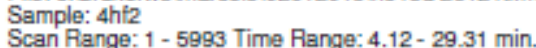

Operator: Marcelo

Date: 2020-03-12 15:08 PM

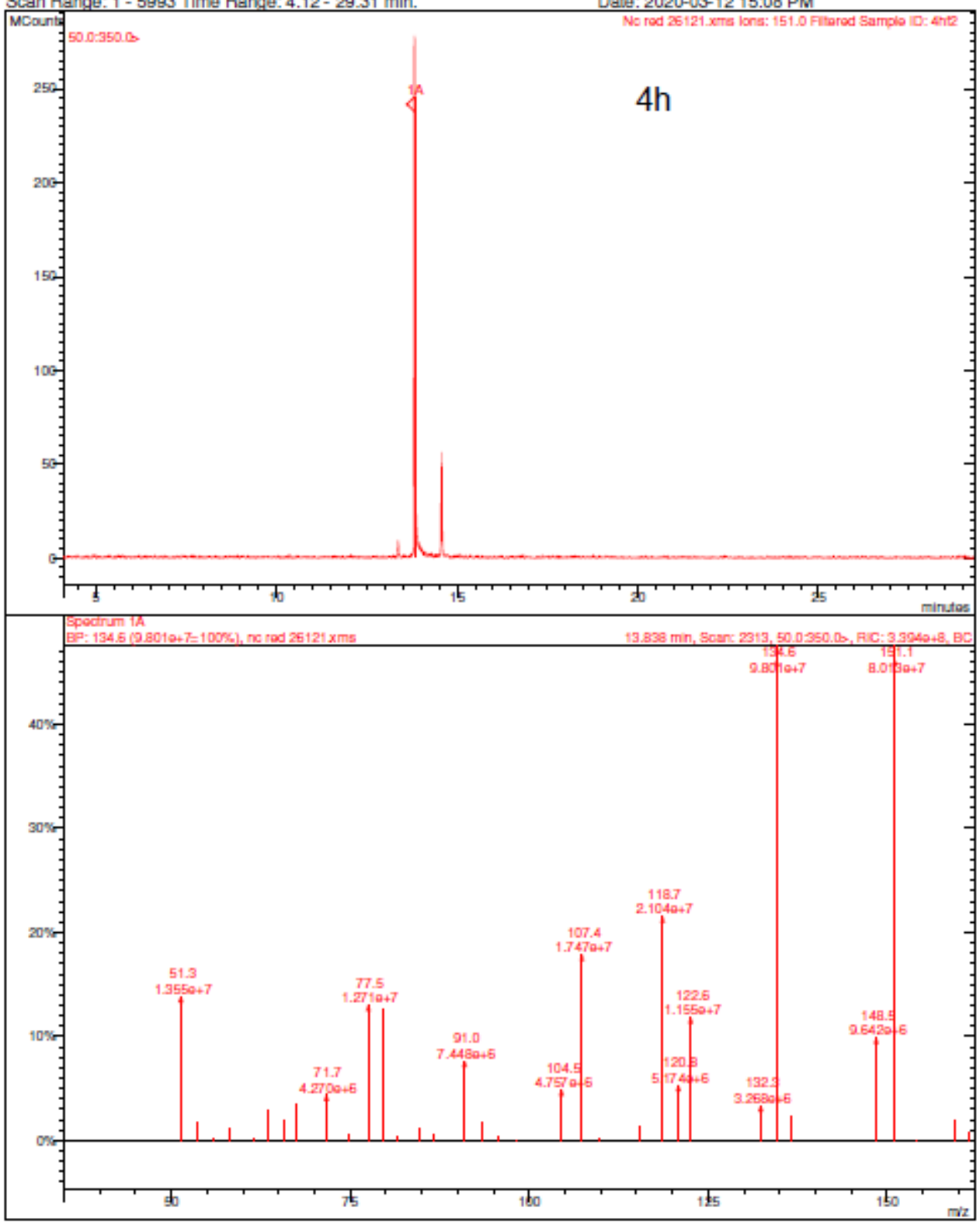


Print Date: 09 Mar 2020 10:27:59

MS Data Review Active Chromatogram and Spectrum Plots - 2020-03-09 10:27 AM File: c:lbrukerwsimarcelol02012019inc red 26069xms Sample: $4 i$

Operator: Marcelo

Scan Range: 1 - 6371 Time Pange: $4.12-25.50$ min

Data: $2020-03-07$ 00:37 AM

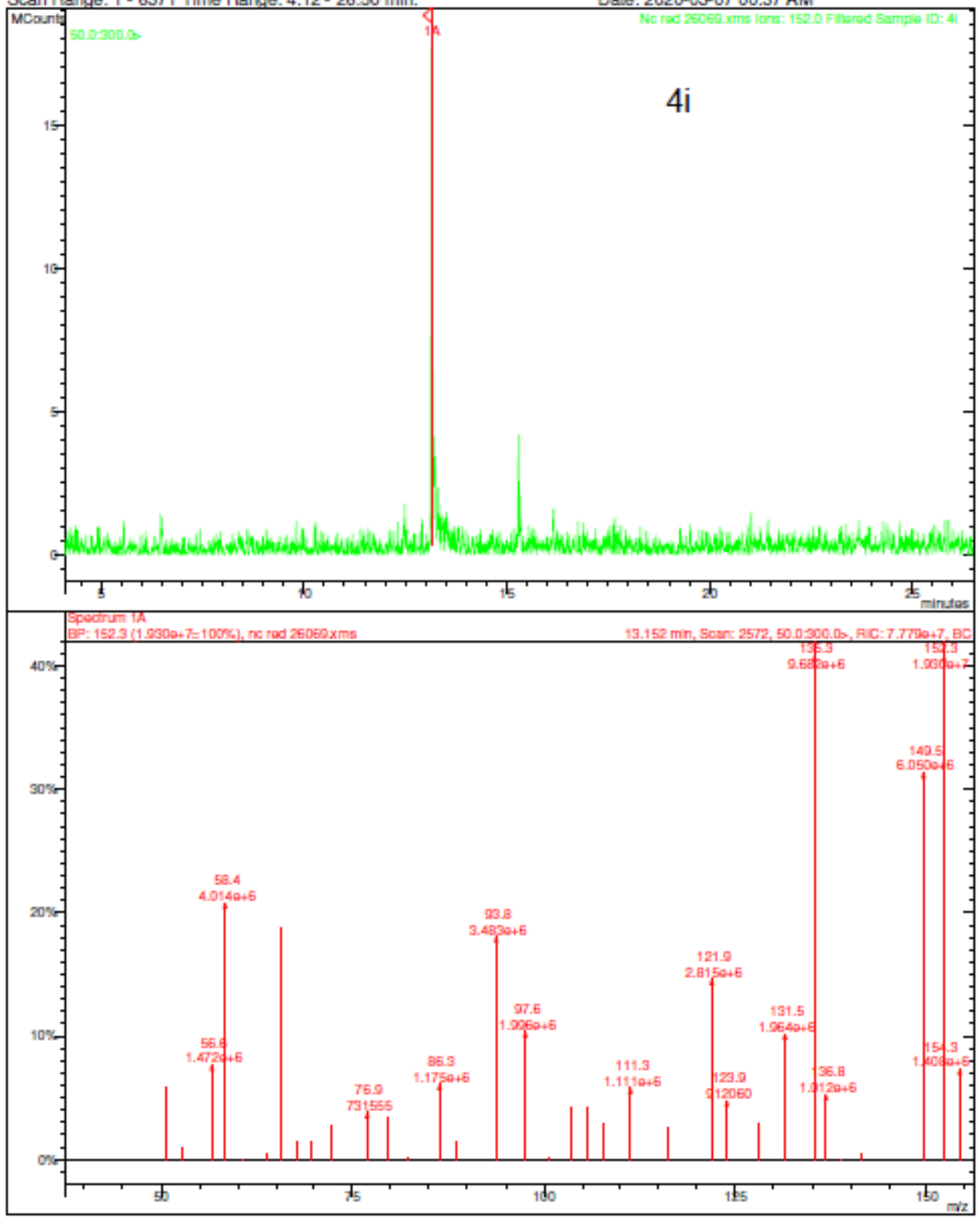


Print Date: 06 Mar 2020 11:03:22

MS Data Review Active Chromatogram and Spectrum Plots - 2020-03-06 11:03 AM

File: c:lbrukerwsimarcelolo2012019inc red 26038xms

Sample: acetone

Operator: Marcelo

Scan Range: 1 - 4321 Time Range: 2.12 - $26.50 \mathrm{~min}$.

Opera: 2020-03-06 06:30 AM

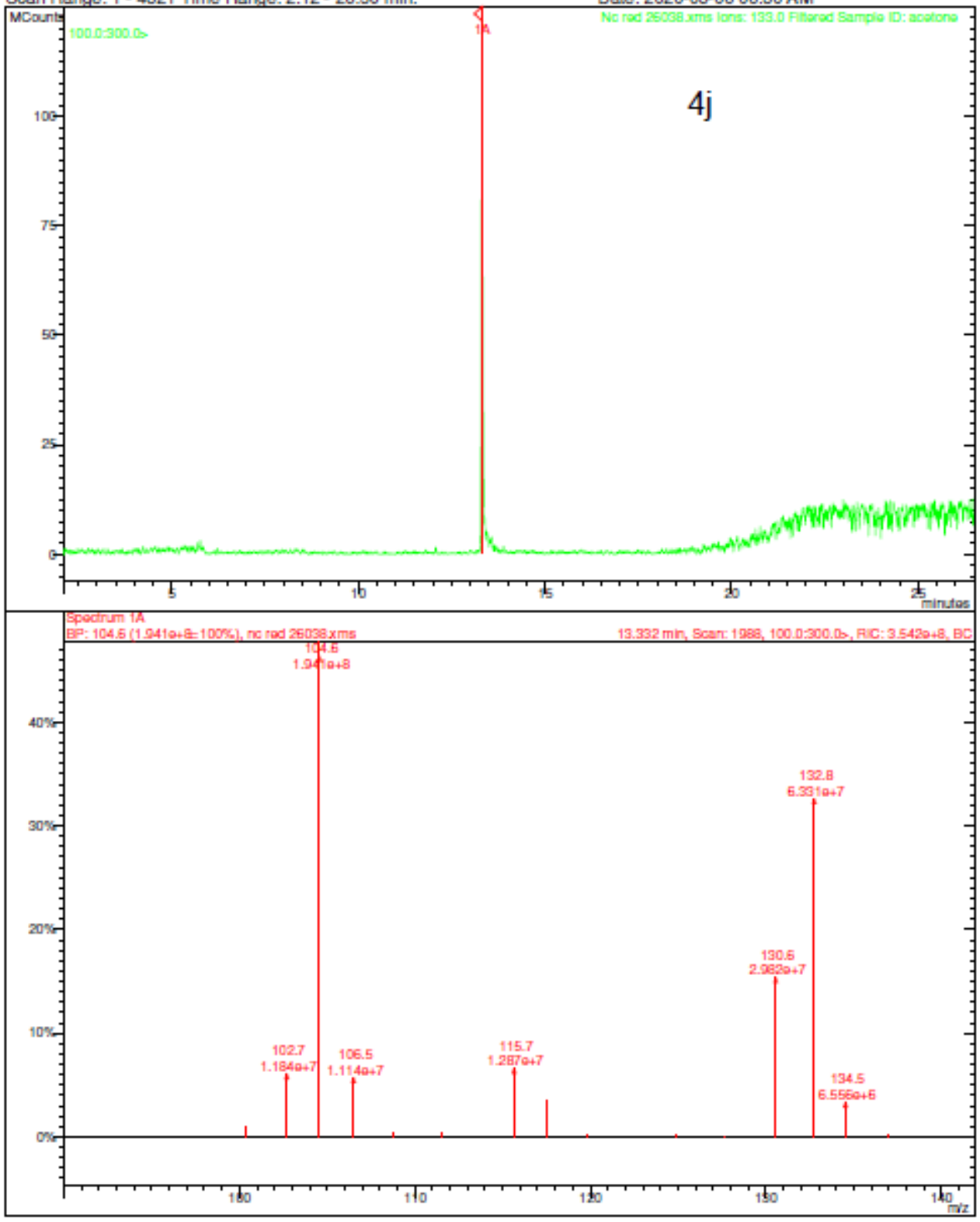


Print Date: 10 Mar 2020 07:57:06

MS Data Review Active Chromatogram and Spectrum Plots - 2020-03-10 07:57 AM

File: c:lbrukerwsimarcelolo2012019inc red 26089xms

Sample: acetone

Operator: Marcelo

Scan Range: 1 -7171 Time Range: 4.12 - 29.31 min. D Date: 2020-03-09 18:21 PM

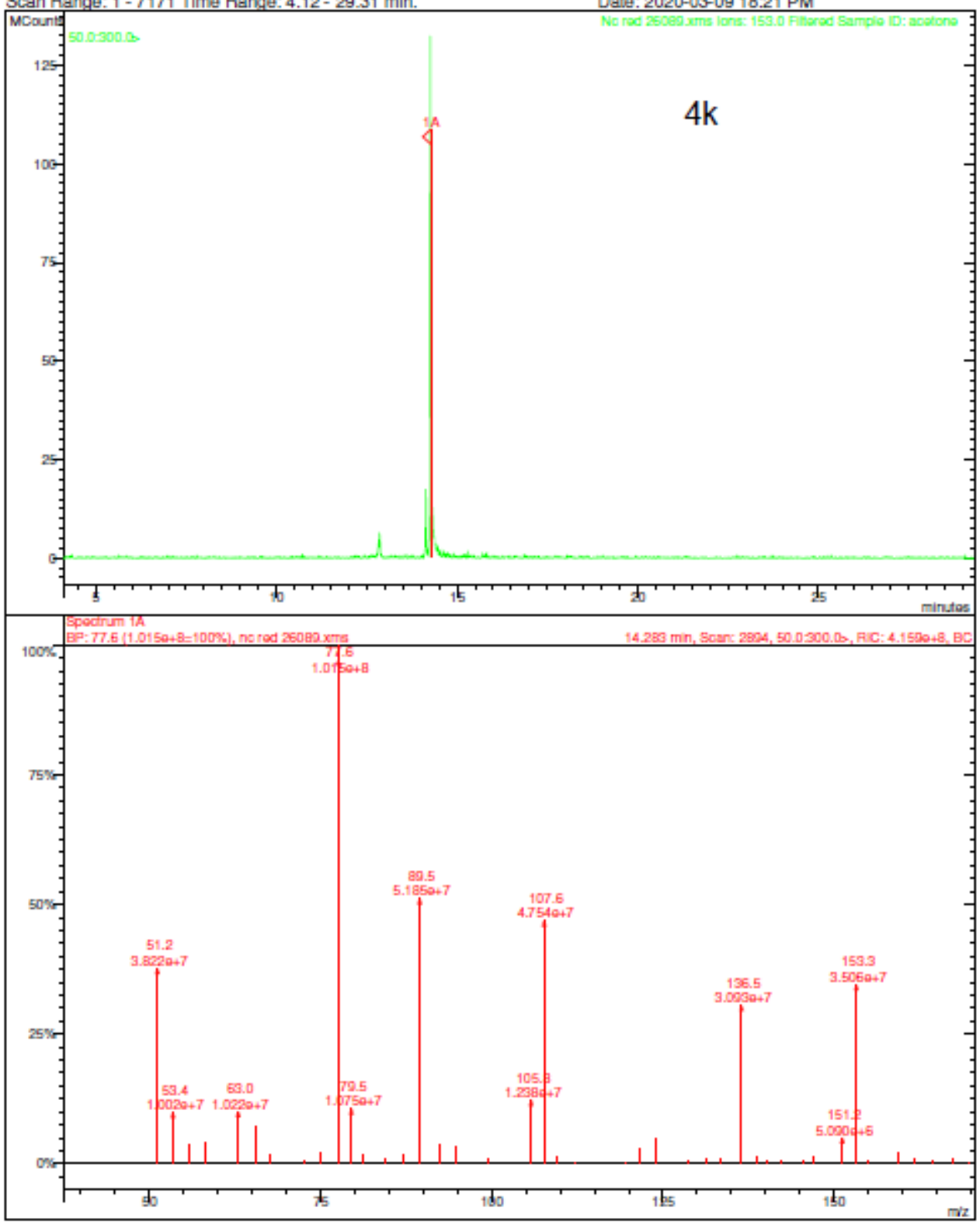


Print Date: 06 Mar 2020 15:07:51

MS Data Review Active Chromatogram and Spectrum Plots - 2020-03-06 15:07 PM File: c: brukerwsimarcelol02012019inc red 26050xms Sample: acatone

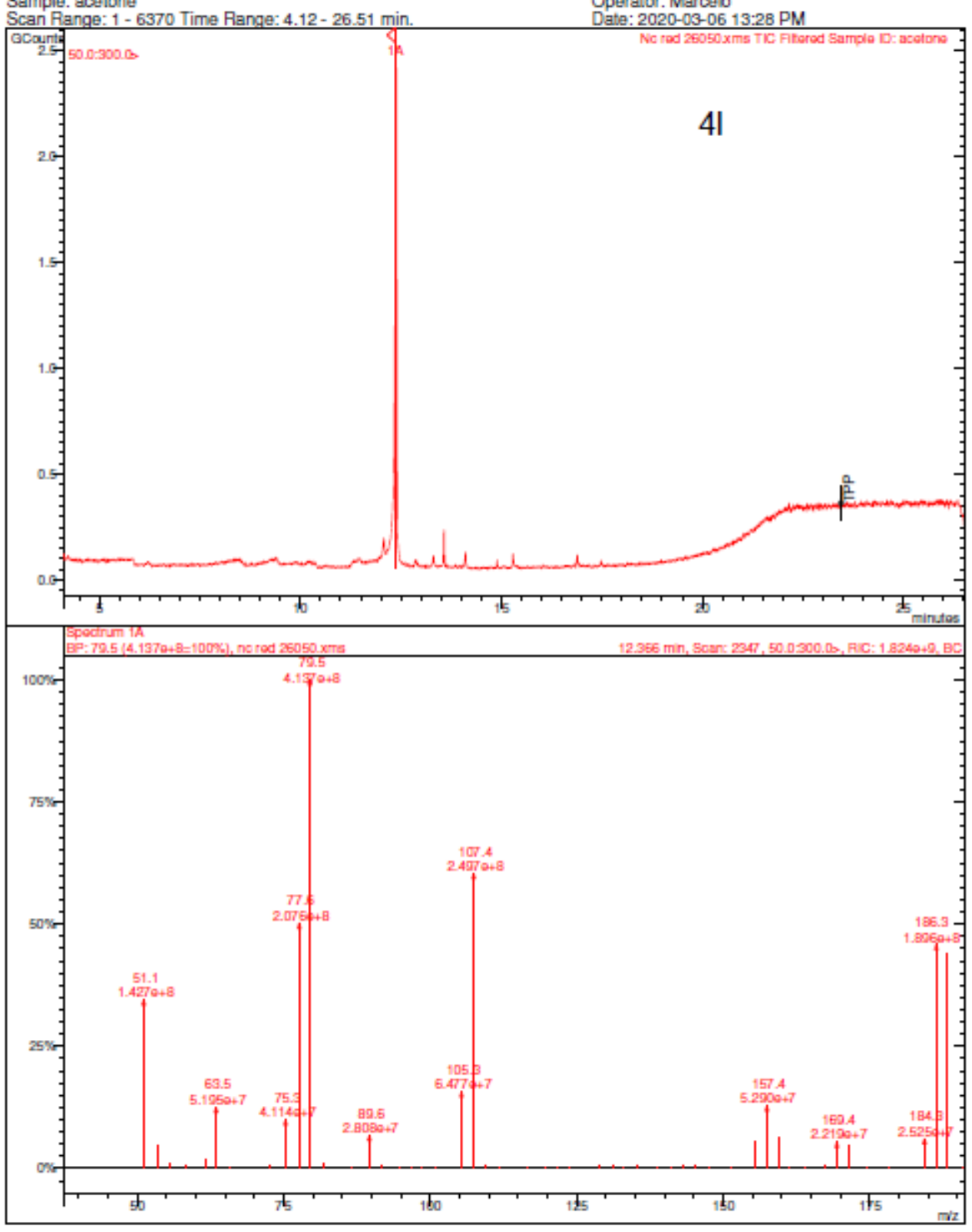

Operator: Marcelo

2020-03-06 13:28 PM 
Print Date: 06 Mar 2020 10:49:48

MS Data Review Active Chromatogram and Spectrum Plots - 2020-03-06 10:49 AM

File: c:.brukerwsimarcelol02012019inc red 26032xms

Sample: acetone

Operator: Marcelo

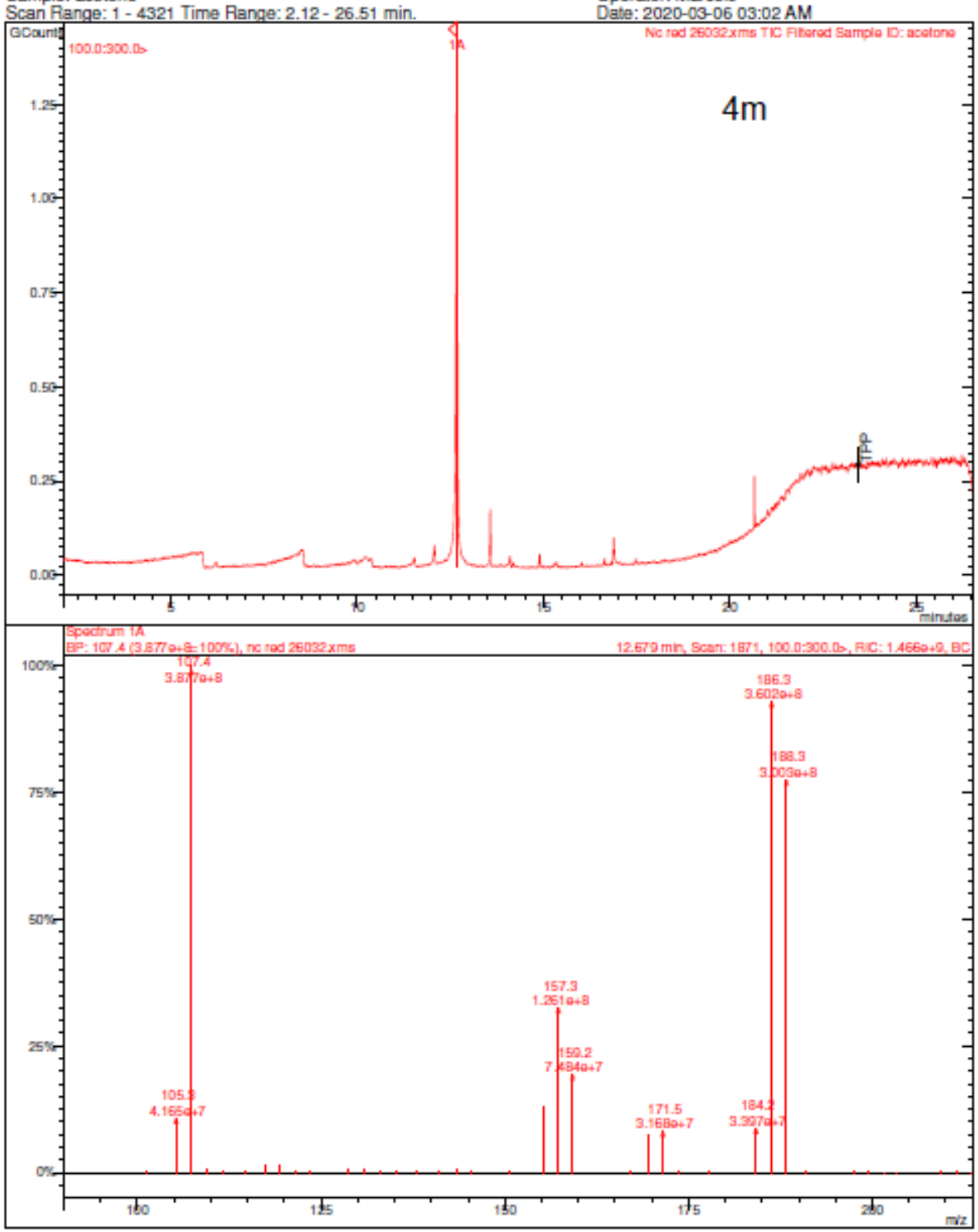


Print Date: 11 Mar 2020 07:59:49

MS Data Review Active Chromatogram and Spectrum Plots - 2020-03-11 07:59 AM

File: c:lbrukerwsimarcelolo2012019inc red 26102.xms $\begin{array}{ll}\text { Sample: sls192-1 } & \text { Operator: Marcalo } \\ \text { Scan Range: } 1 \text { - 7166 Time Range: } 4.12 \text { - } 29.30 \mathrm{~min} . & \text { Date: 2020-03-10 }\end{array}$

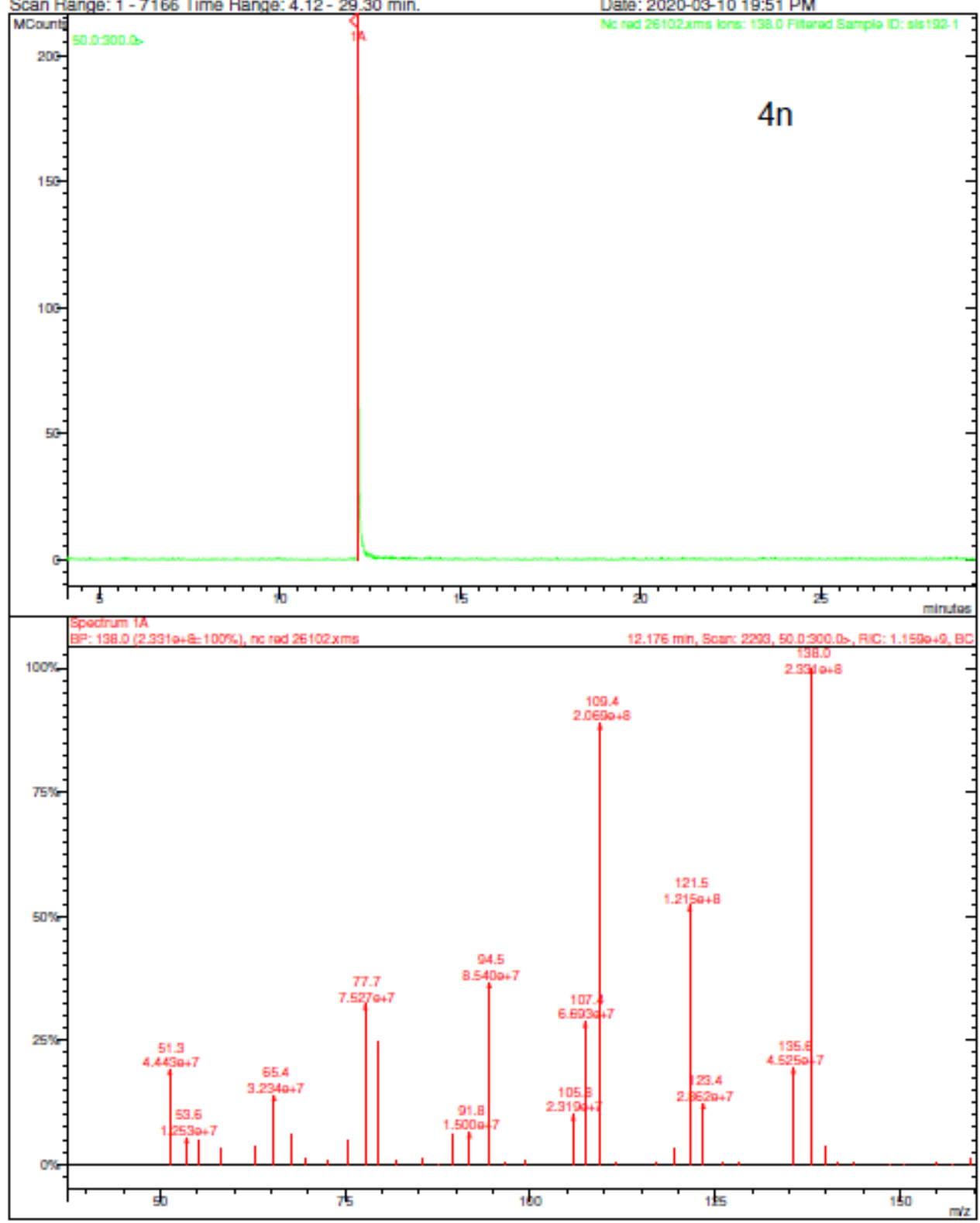


Print Date: 06 Mar 2020 11:05:00

MS Data Review Active Chromatogram and Spectrum Plots - 2020-03-06 11:04 AM

File: c:lbrukerwsimarcelol02012019inc red 26040 xms

Sample: acetone

Scan Range: 1 - 4321 Time Range: 2.13 - 26.51 min.

perator: Marcelb

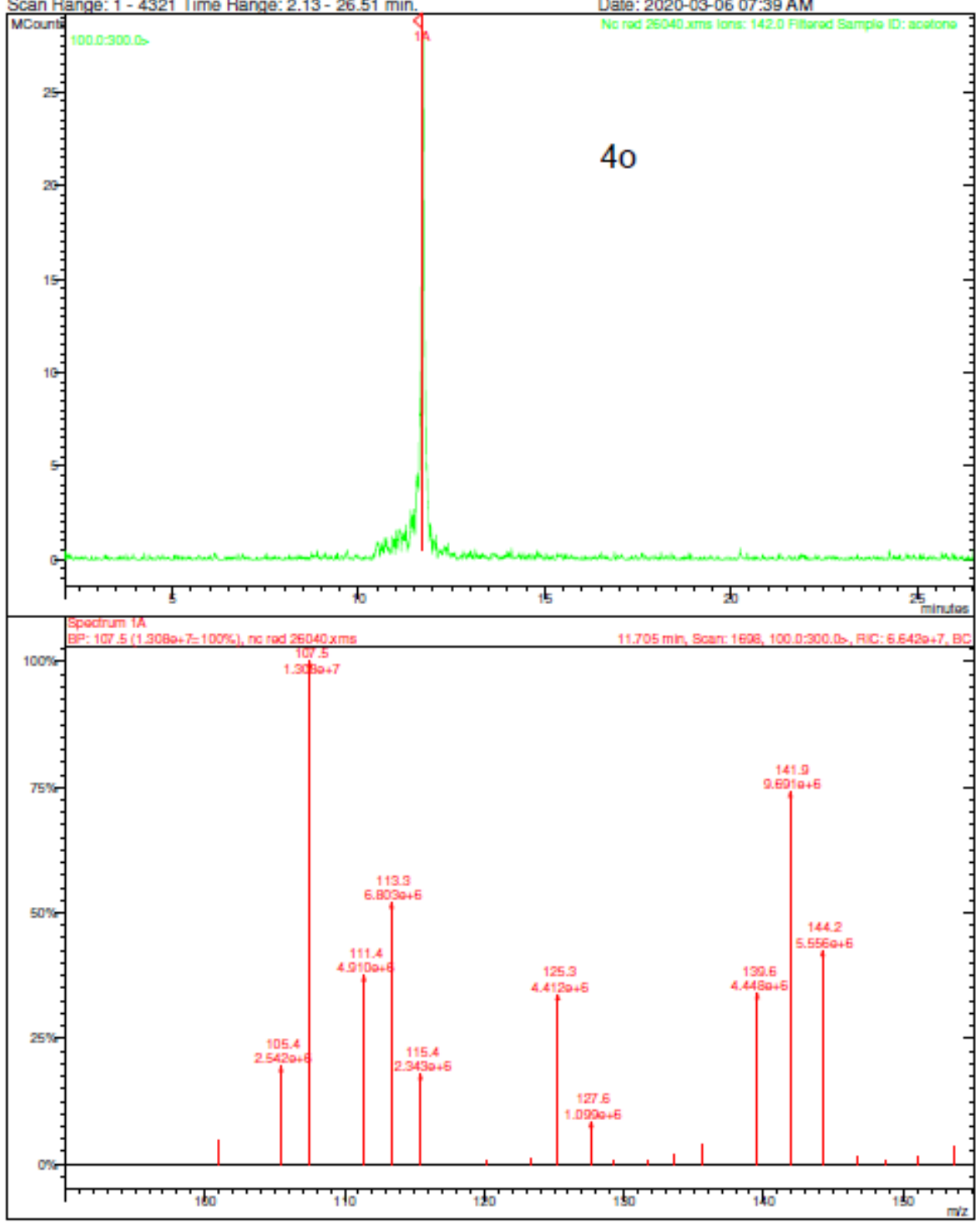


MS Data Review Active Chromatogram and Spectrum Plots - 2020-03-06 11:07 AM File: c.'brukerws imarcelolo2012019inc red 26041 xms Sample: sls178
Scan Range: 1 - 4321 Time Range: 2.12 - 26.51 min.

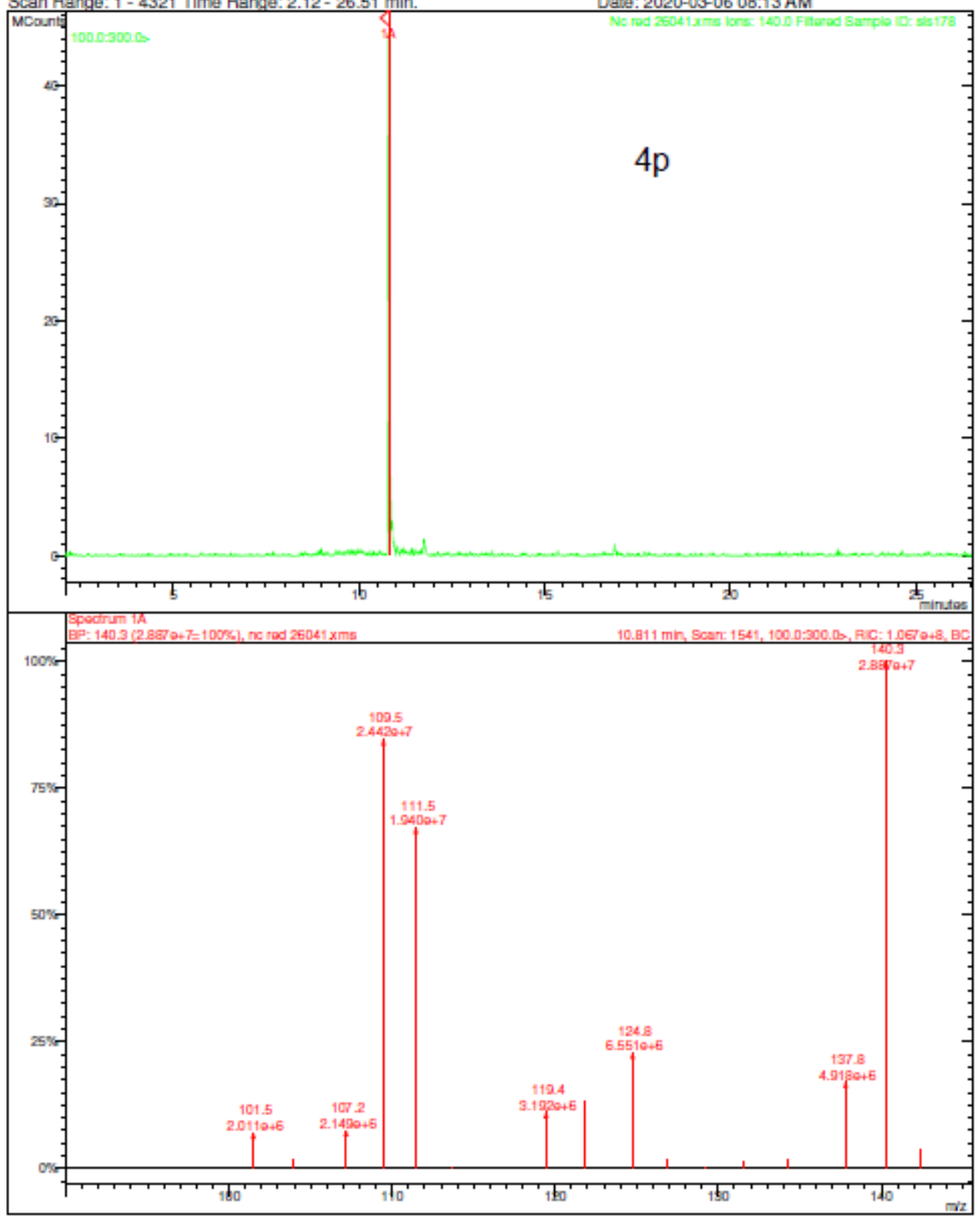


Print Date: 06 Mar 2020 10:59:53

MS Data Review Active Chromatogram and Spectrum Plots - 2020-03-06 10:59 AM

File: c:lbrukerwsimarcelolo2012019inc red 26034xms

Sample: acetone

Operator: Marcelo

Scan Range: 1 - 4321 Time Range: 2.12 - 26.50 min. Date: 2020-03-06 04:11 AM

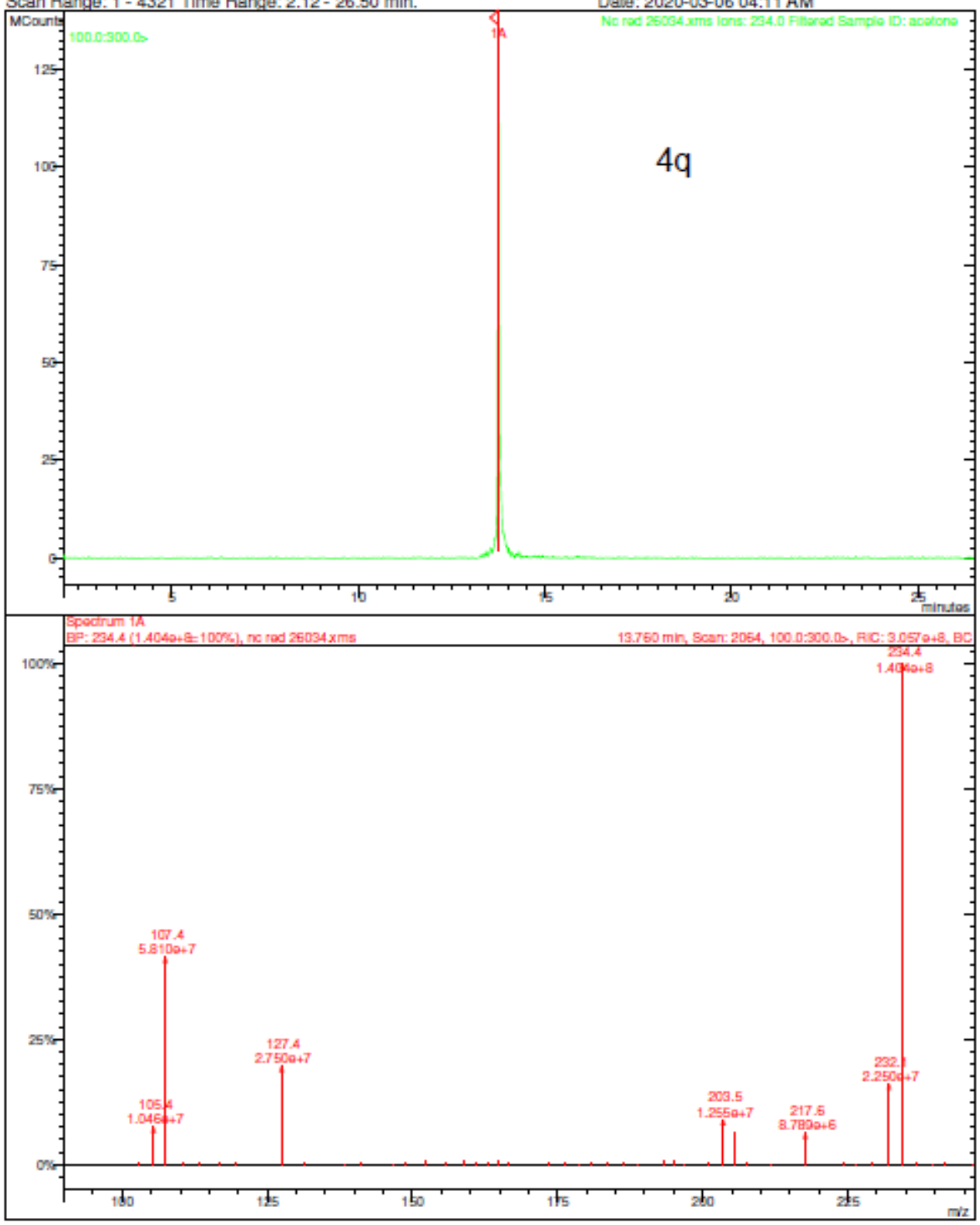


Print Date: 09 Mar 2020 10:17:40

MS Data Review Active Chromatogram and Spectrum Plots - 2020-03-09 10:17 AM

File: c:lbrukerwsimarcelolo2012019inc red $26057 \times \mathrm{ms}$

Sample: $5 a$

Operator: Marcelo

Scan Range: 1 - 6371 Time Range: 4.12 - 26.50 min

Date: 2020-03-06 17:37 PM

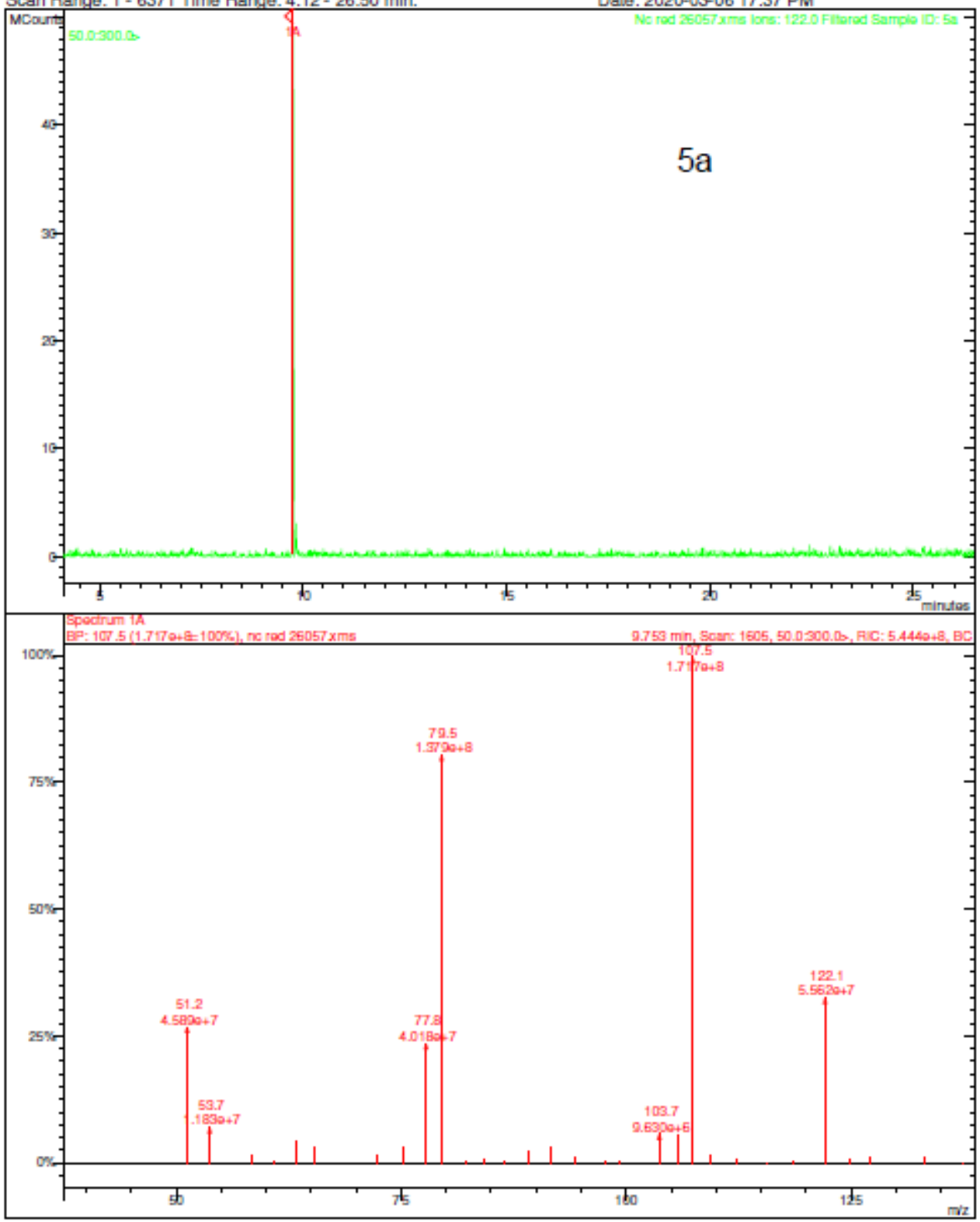


Print Date: 06 Mar 2020 10:03:26

MS Data Review Active Chromatogram and Spectrum Plots - 2020-03-06 10:03 AM

File: c:lbrukerwsimarcelolo2012019inc red 26028xms

Sample: acetone

Operator: Marcelo

Scan Range: 1 - 4321 Time Range: 2.12 - 26.50 min

Opto: $2020-03-0600: 42$ AM
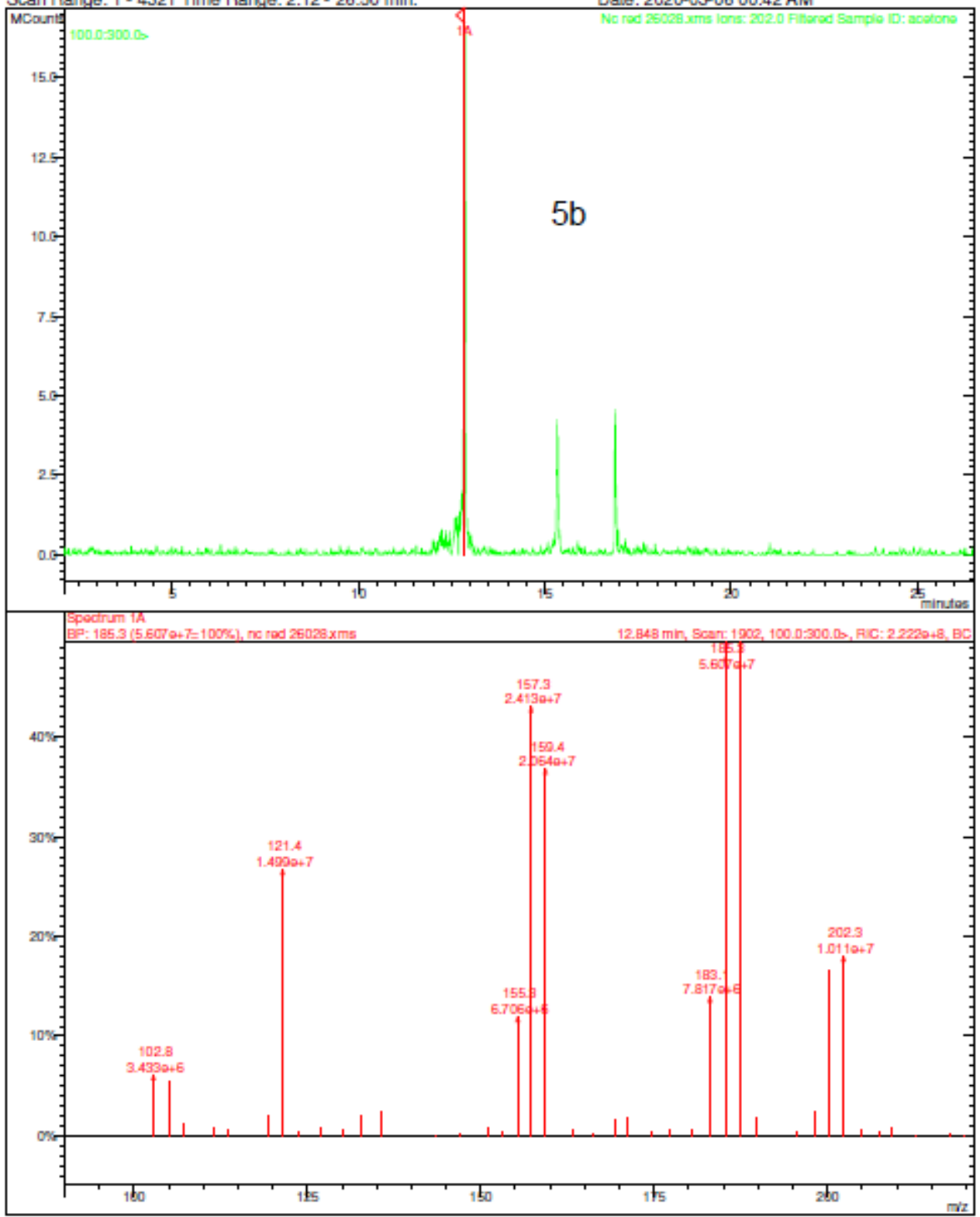
MS Data Review Active Chromatogram and Spectrum Plots - 2020-03-09 10:21 AM

File: c:lbrukerwsimarcelolo2012019inc red 26061 xms Sample: sls 181

Operator: Marcalo

Scan Range: 1 - 6371 Time Range: 4.12 - 26.50 min

Operator: Marcolo $10.58 \mathrm{PM}$

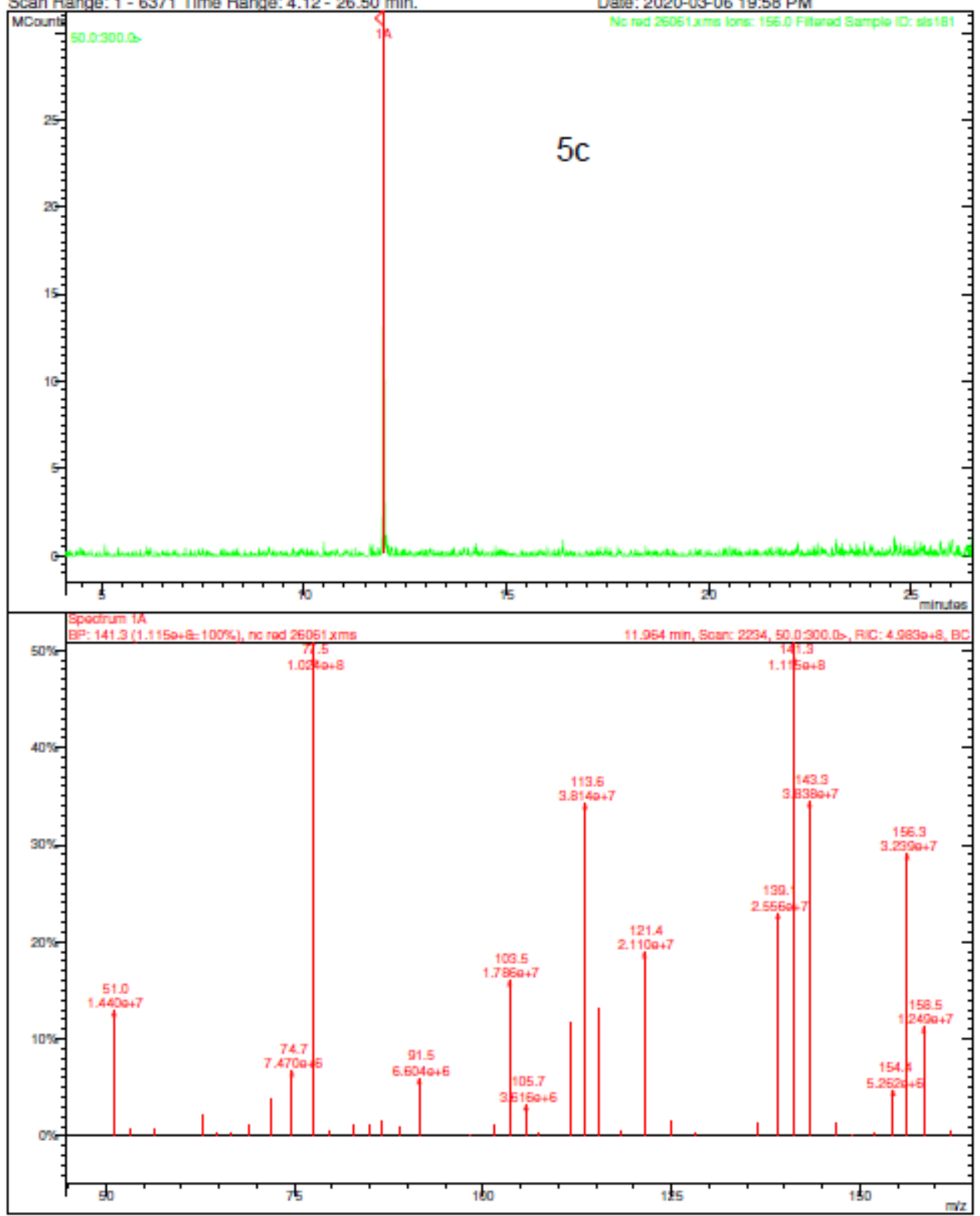


Print Date: 06 Mar 2020 15:09:57

MS Data Review Active Chromatogram and Spectrum Plots - 2020-03-06 15:09 PM

File: c:lbrukerwsimarcelol02012019inc red $26051 \mathrm{xms}$

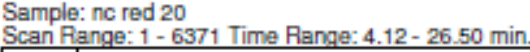

(ont

Operator: Marcab

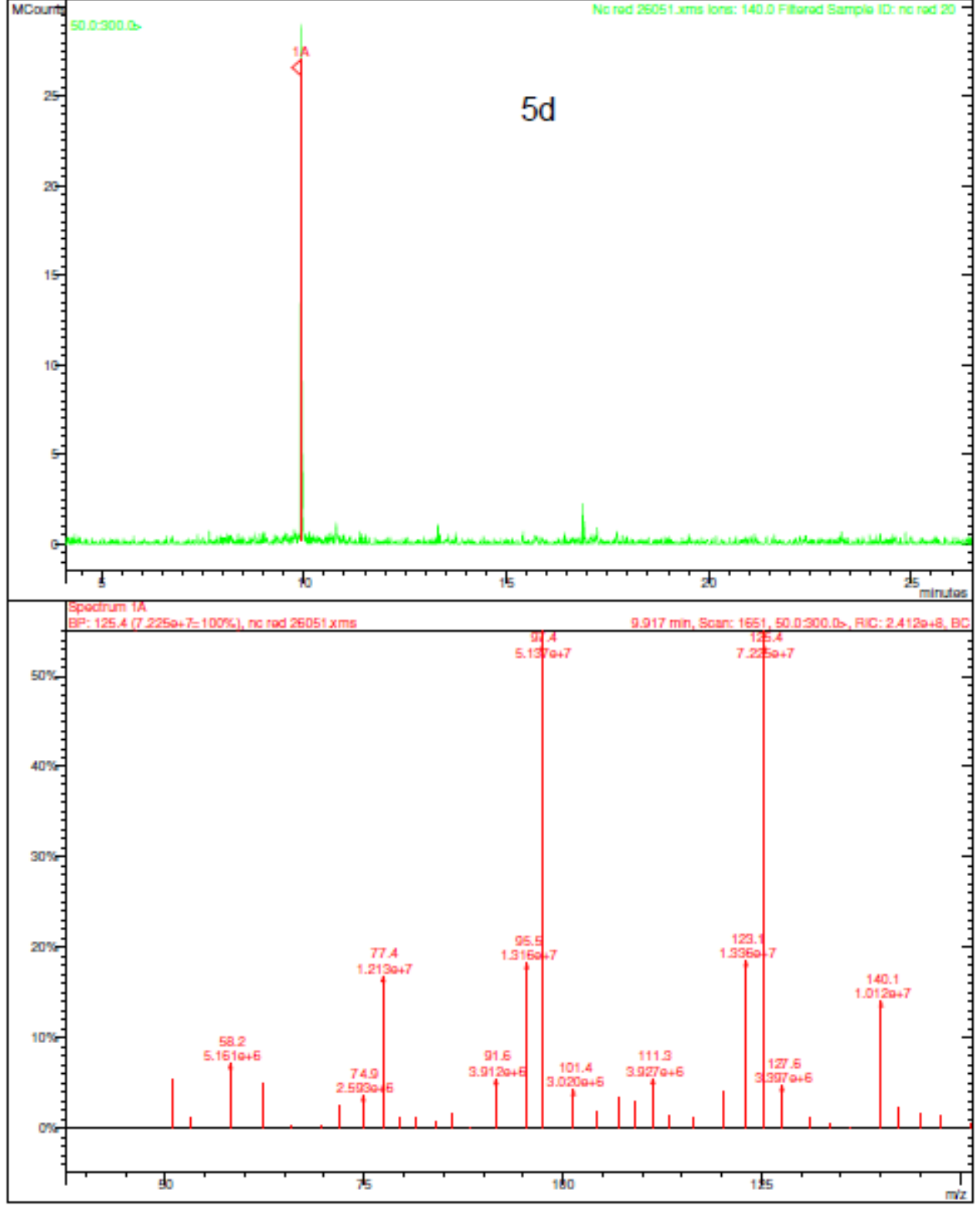


Print Date: 10 Mar 2020 15:35:27

MS Data Review Active Chromatogram and Spectrum Plots - 2020-03-10 15:35 PM File: c:lbrukerwsimarcelol02012019inc red 26093xms Sample: sls194 Scan Range: 1 - 7166 Time Range: 4.12 - 29.30 min Scan Ran
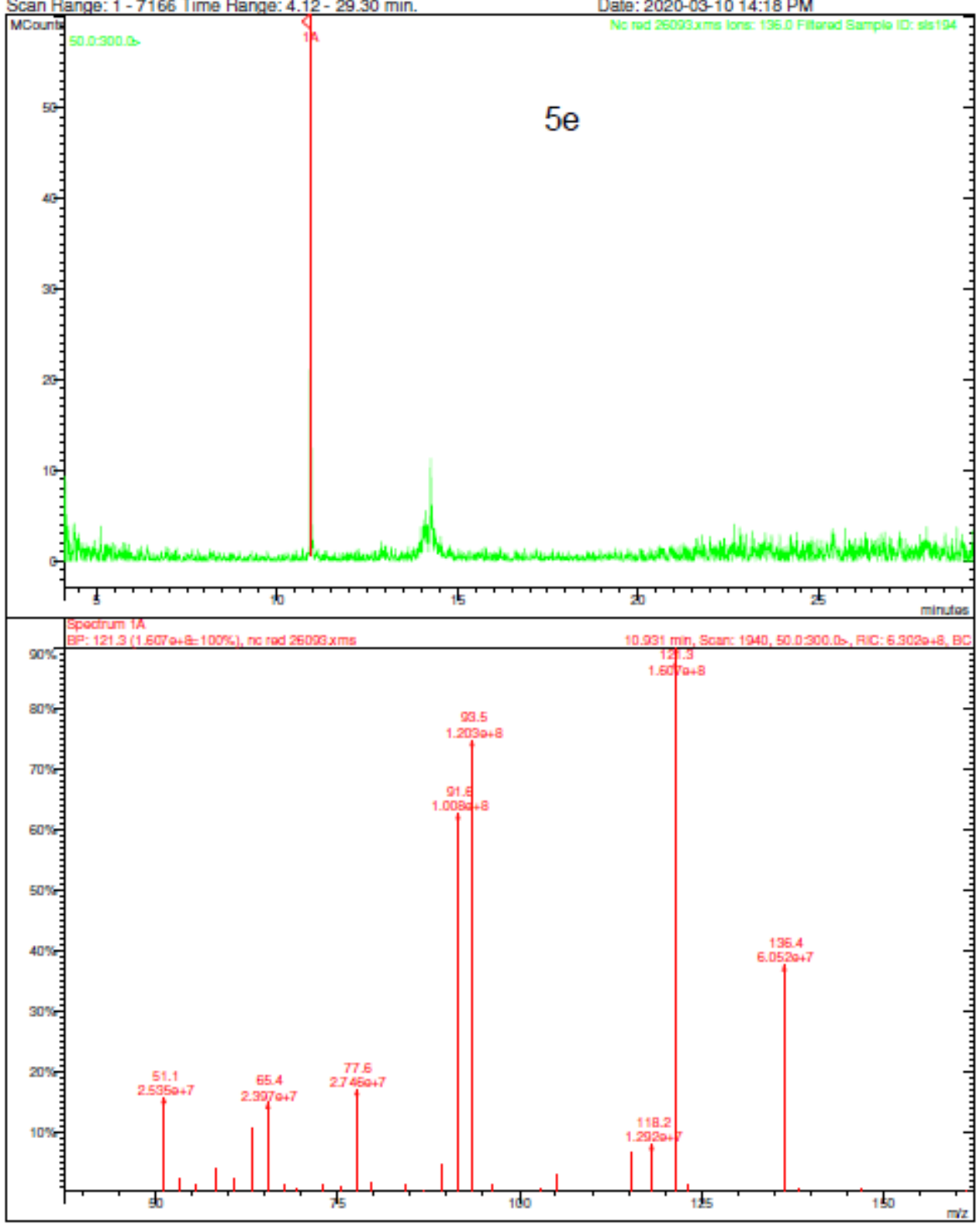
Print Date: 10 Mar 2020 07:58:48

MS Data Review Active Chromatogram and Spectrum Plots - 2020-03-10 07:58 AM

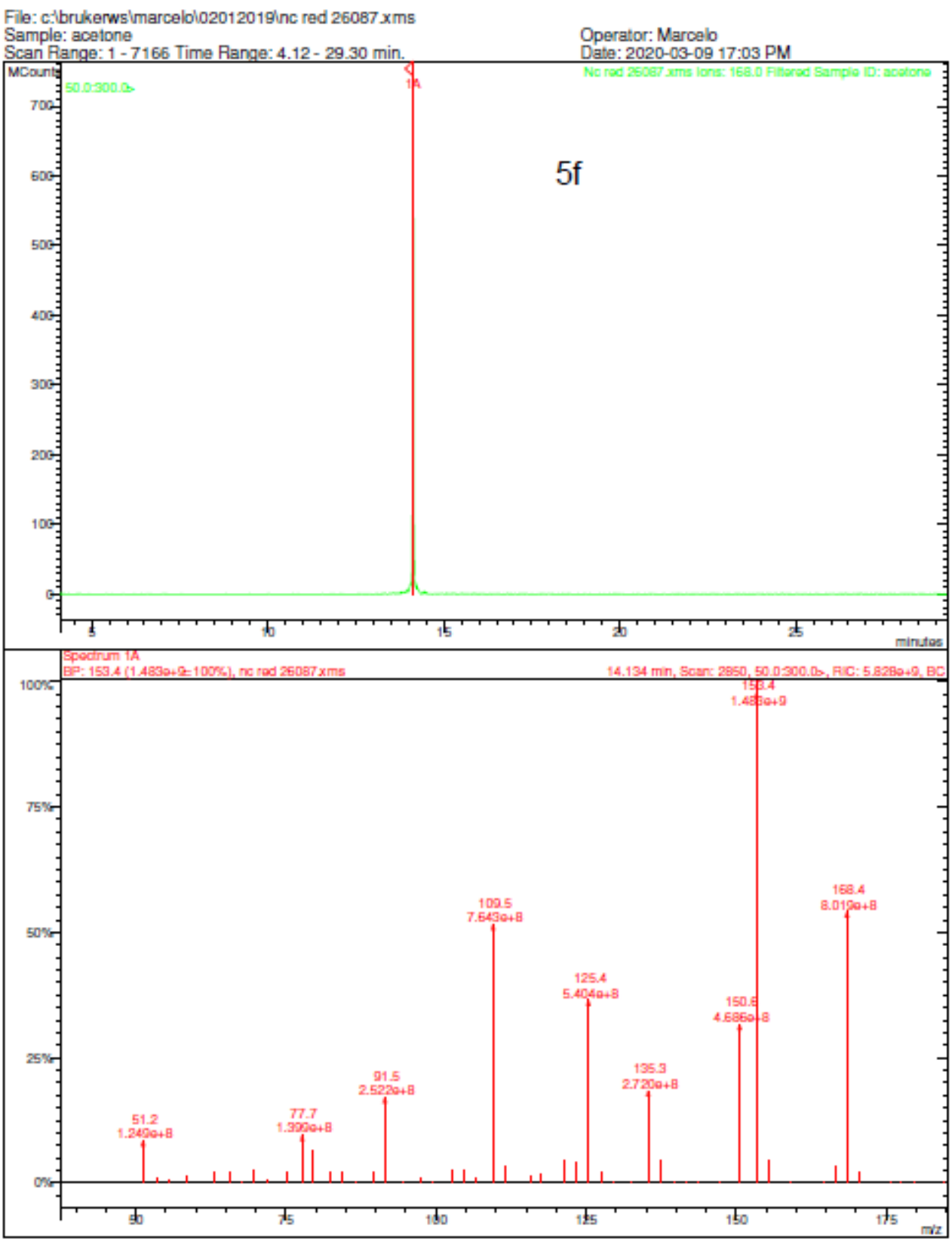


PIIn Laie: Uy mar ZUzU 10.32.11

MS Data Review Active Chromatogram and Spectrum Plots - 2020-03-09 10:32 AM

File: c.'brukerwsimarcelolo2012019inc red $26074 \times \mathrm{ms}$ Sample: acetone Operator: Marcelo

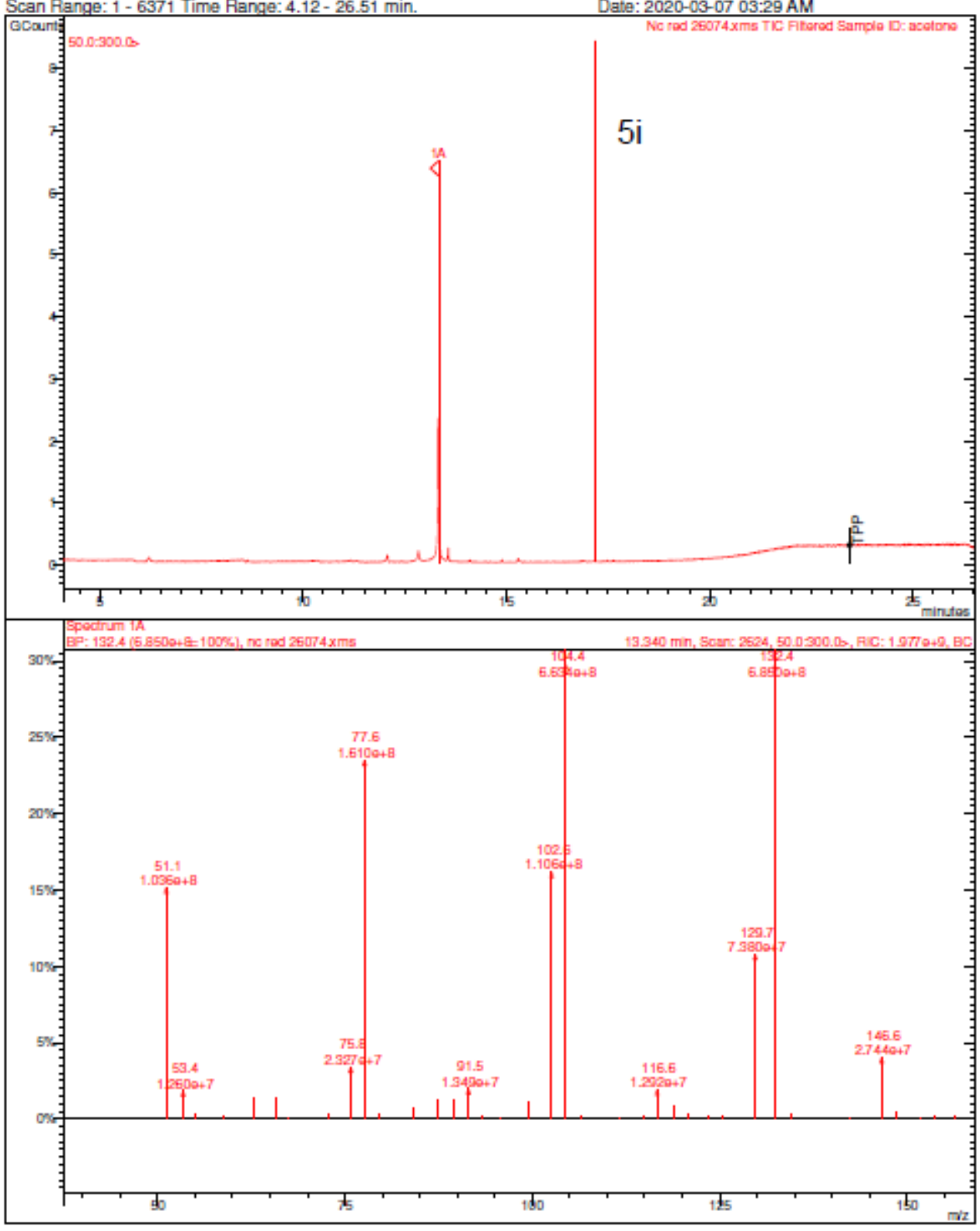


Print Date: 09 Mar 2020 14:20:37

MS Data Review Active Chromatogram and Spectrum Plots - 2020-03-09 14:20 PM

File: c:lbrukerwsimarcelol02012019inc red 26082.xms Sample: acetone

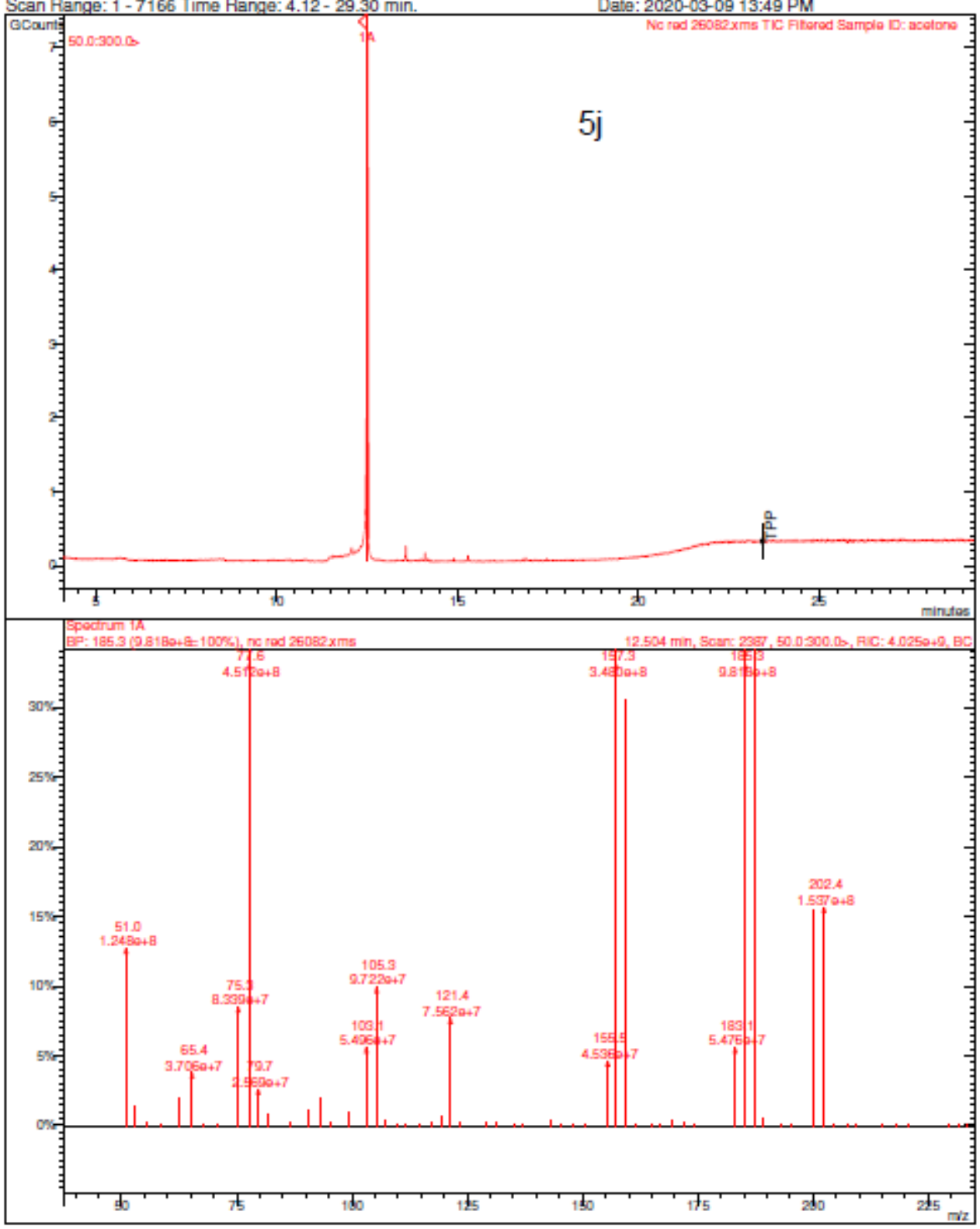

Operator: Marcelo

: $2020-03-09$ 13:49 PM 
Print Date: 09 Mar 2020 10:34:35

MS Data Review Active Chromatogram and Spectrum Plots - 2020-03-09 10:34 AM

File: c:lbrukerwsimarcelolo2012019inc red 26075xms

Sample: $5 k$

Scan Range: 1 - 6371 Time Range: 4.12 - $26.51 \mathrm{~min}$.

Operator: Marcelo

Date. 2020-03-07 04:04 AM

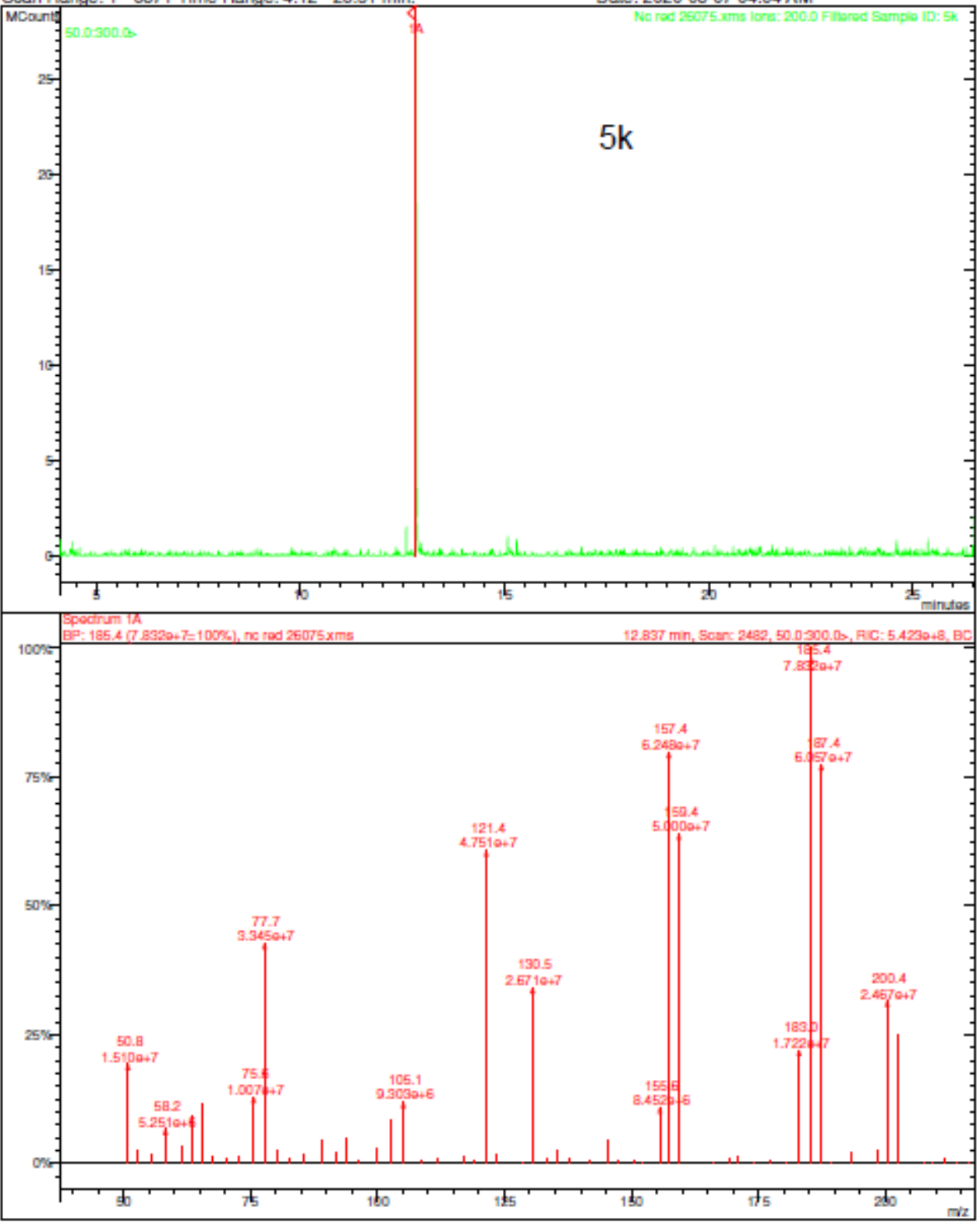


Print Date: 06 Mar 2020 09:57:39

MS Data Review Active Chromatogram and Spectrum Plots - 2020-03-06 09:56 AM

File: c:lbrukerwsimarcelol02012019inc red 26023xms

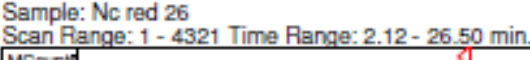
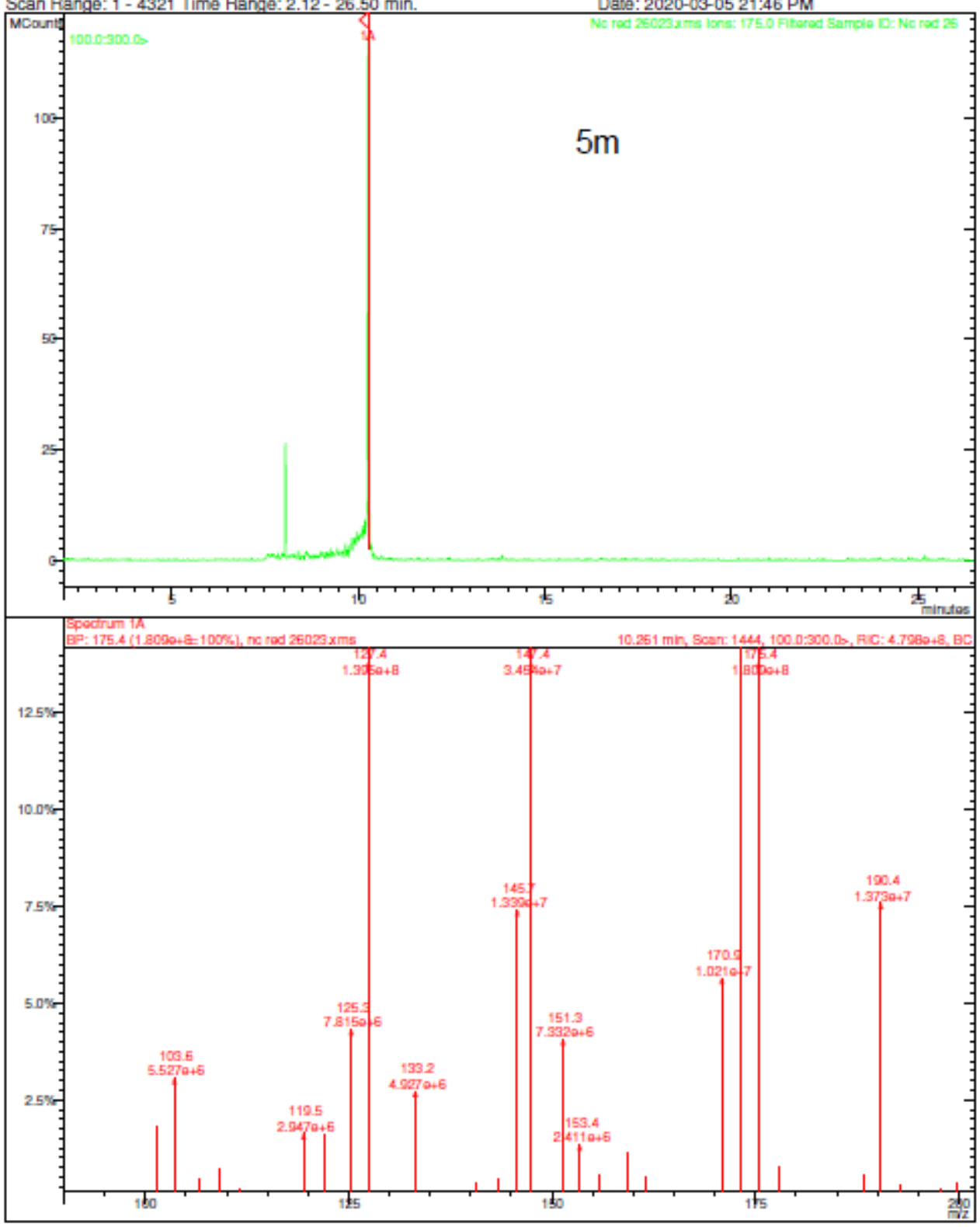
Print Date: 11 Mar 2020 08:01:33

MS Data Review Active Chromatogram and Spectrum Plots - 2020-03-11 08:01 AM File: c:lbrukerwsimarcelolo2012019inc red $26104 \mathrm{xms}$ Sample: sls193 2 . MCount

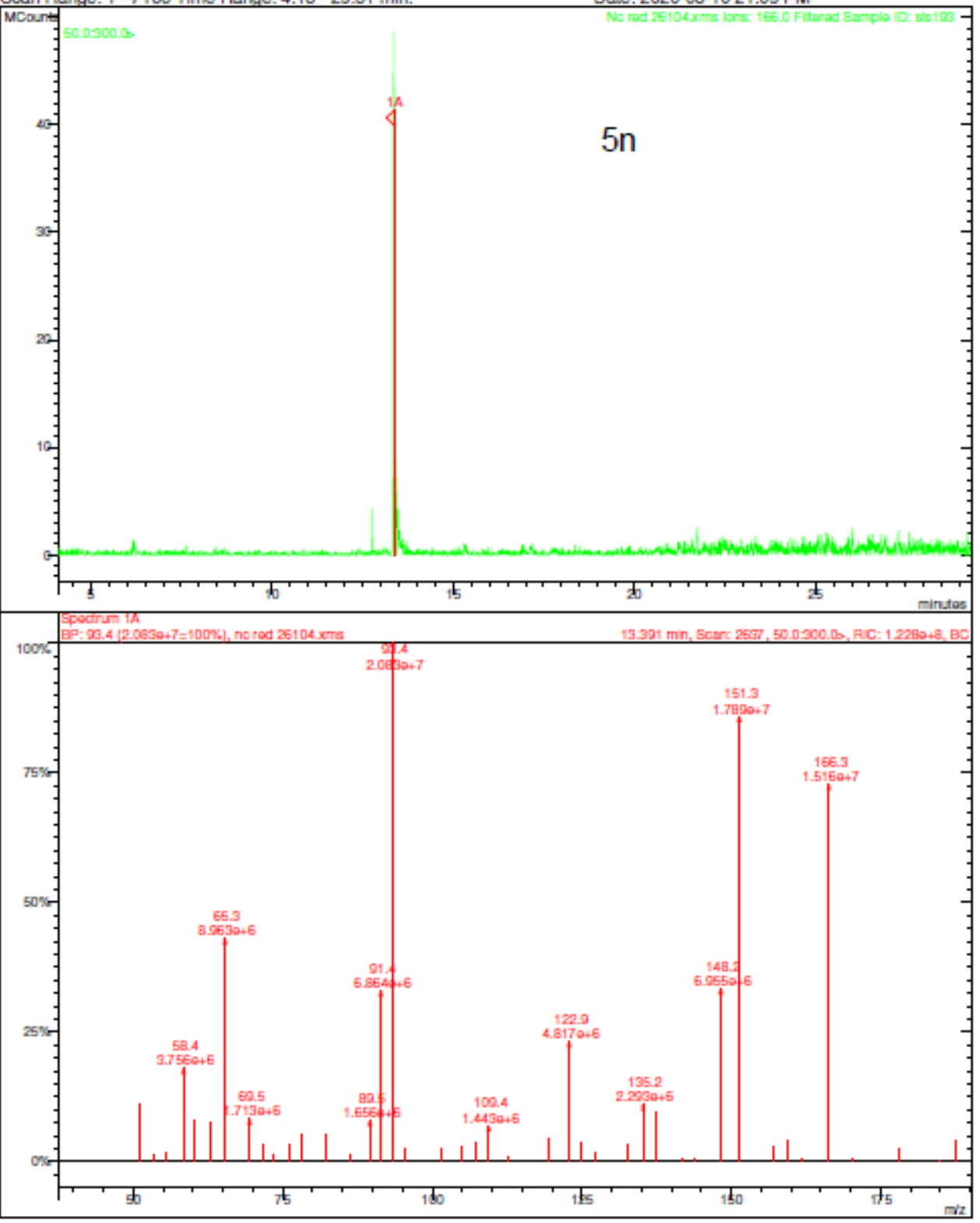


Print Date: 09 Mar 2020 10:15:54

MS Data Review Active Chromatogram and Spectrum Plots - 2020-03-09 10:15 AM

File: c.lbrukerwsimarcelolo2012019inc red 26056xms Sample: acetone

Operator: Marcelo

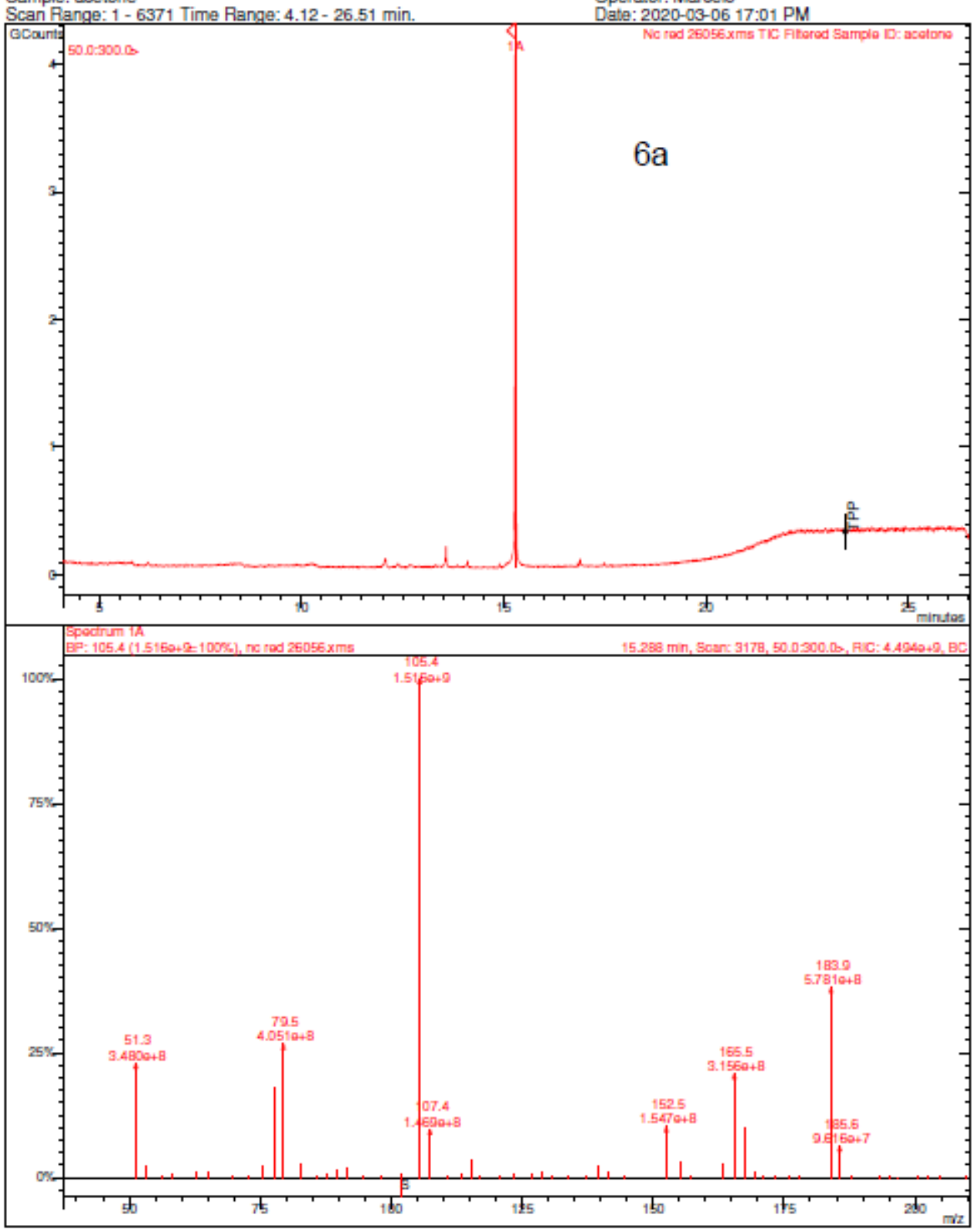


Print Date: 11 Mar 2020 16:46:55

MS Data Review Active Chromatogram and Spectrum Plots - 2020-03-11 16:46 PM File: c: ibrukerwsimarcelolo2012019inc red 26110xms Sample: sls191 rec Operator: Marcalo Scan Range: 1 - 5989 Time Range: 4.12 - 29.31 min.

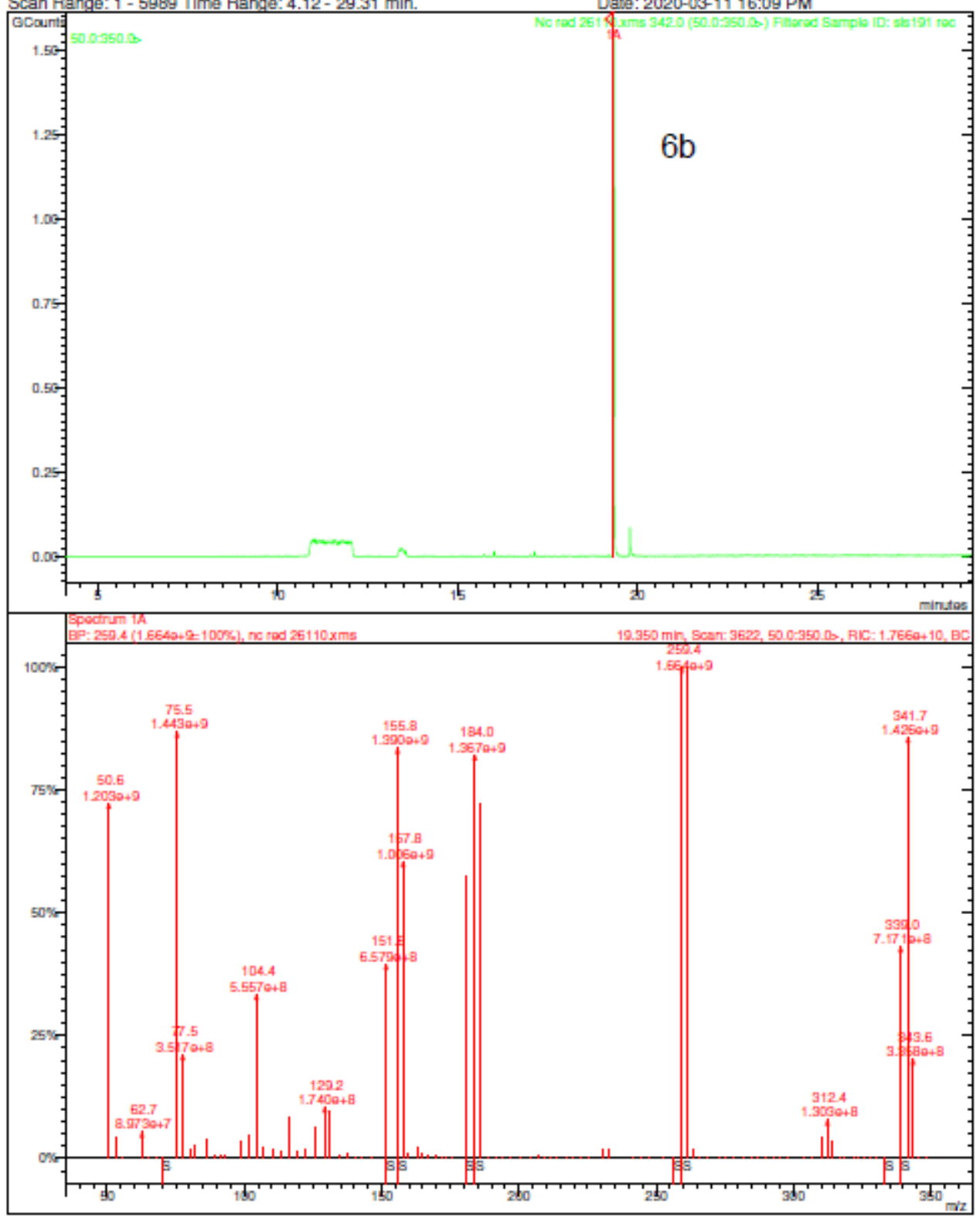


Print Date: 06 Mar 2020 09:59:54

MS Data Review Active Chromatogram and Spectrum Plots - 2020-03-06 09:59 AM

File: c:brukerwsimarcelolo2012019inc red 26025xms

Sample: MA 85

Operator: Marcalo

Scan Range: 1 - 4321 Time Range: 2.12 - 26.50 min

Operator. Marcalo 57.57 PM

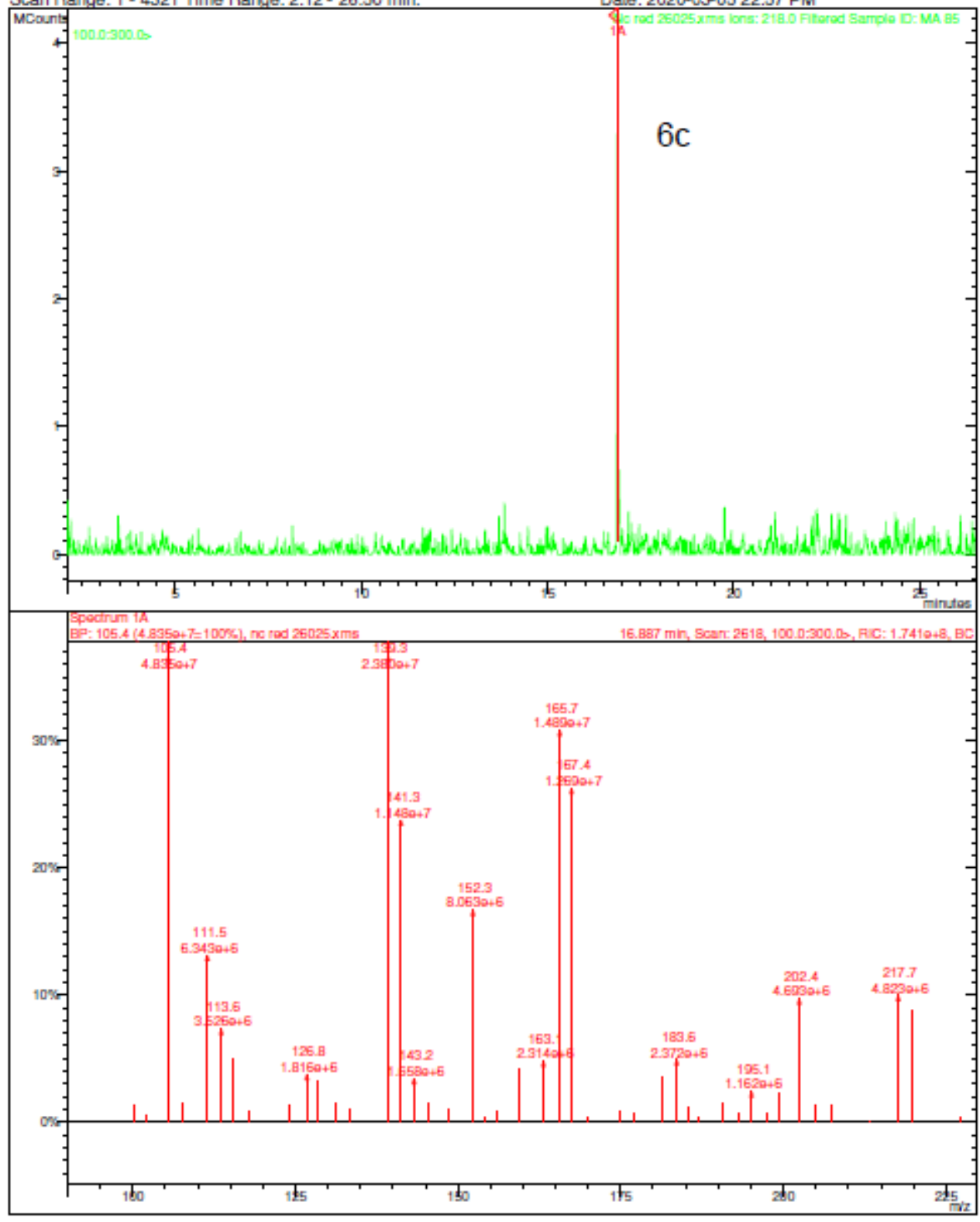


Print Date: 06 Mar 2020 11:11:12

MS Data Review Active Chromatogram and Spectrum Plots - 2020-03-06 11:10 AM

File: c:lbrukerwsimarcelolo2012019inc red 26044 Xms

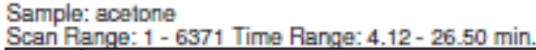

Operator: Marcalo

Opto: $2020-03-0609: 58$ AM

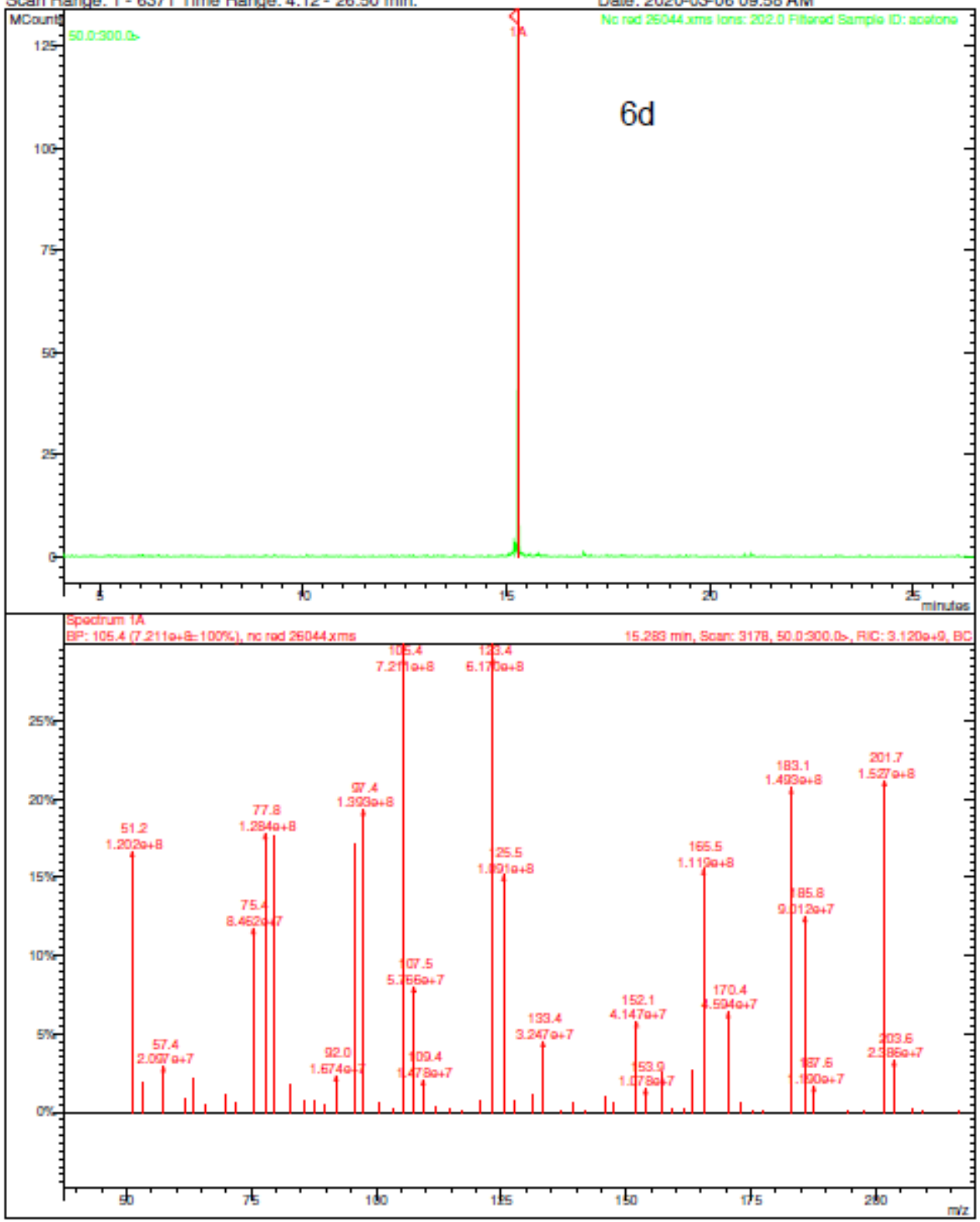

